\title{
ADAPTIVE WEAK APPROXIMATION OF REFLECTED AND STOPPED DIFFUSIONS
}

\author{
CHRISTIAN BAYER, ANDERS SZEPESSY, AND RAÚL TEMPONE
}

\begin{abstract}
We study the weak approximation problem of diffusions, which are reflected at a subset of the boundary of a domain and stopped at the remaining boundary. First, we derive an error representation for the projected Euler method of Costantini, Pacchiarotti and Sartoretto [Costantini et al., SIAM J. Appl. Math., 58(1):73-102, 1998], based on which we introduce two new algorithms. The first one uses a correction term from the representation in order to obtain a higher order of convergence, but the computation of the correction term is, in general, not feasible in dimensions $d>1$. The second algorithm is adaptive in the sense of Moon, Szepessy, Tempone and Zouraris [Moon et al., Stoch. Anal. Appl., 23:511-558, 2005], using stochastic refinement of the time grid based on a computable error expansion derived from the representation. Regarding the stopped diffusion, it is based in the adaptive algorithm for purely stopped diffusions presented in Dzougoutov, Moon, von Schwerin, Szepessy and Tempone [Dzougoutov et al., Lect. Notes Comput. Sci. Eng., $44,59-88,2005]$. We give numerical examples underlining the theoretical results.
\end{abstract}

\section{Contents}

\begin{tabular}{|lr|}
\hline $1 . \quad$ Introduction & 1 \\
\hline $2 . \quad$ Approximation of stopped reflected diffusions & 4 \\
\hline 3. An error expansion & 7 \\
\hline $4 . \quad$ The adaptive algorithm for reflected diffusions & 15 \\
\hline 5. The adaptive algorithm for stopped reflected diffusions & 25 \\
\hline $6 . \quad$ The algorithm with correction term for purely reflecting diffusions & 27 \\
\hline 7. Numerical examples & 32 \\
\hline $7.1 . \quad$ A reflected diffusion in dimension 1 & 33 \\
\hline 7.2. A purely reflected diffusion in dimension 2 & 37 \\
\hline 7.3. A stopped reflected diffusion in dimension 2 & 39 \\
\hline 8. Conclusions & 43 \\
\hline References & 44 \\
\hline Appendix A. An upper bound for hitting probabilities & 45
\end{tabular}

\section{INTRODUCTION}

In this report, we present two new algorithms for the approximation of reflected diffusions, which are possibly stopped when hitting a certain part of the boundary of the domain. From a numerical point of view, these stochastic differential equations are interesting because they provide stochastic representations for (suitable) parabolic PDEs with Neumann boundary conditions. Moreover, certain applications in financial mathematics also exist, e.g., in the context of look-back options.

Since stopped diffusions are more prominent in the literature, let us focus on the reflected diffusion first. Let $B=\left(B_{t}\right)_{t \in[0, \infty[}$ be a $d$-dimensional Brownian motion defined on the stochastic basis $\left(\Omega, \mathcal{F},\left(\mathcal{F}_{t}\right)_{t \in[0, \infty[}, P\right)$. Informally, a reflected diffusion will be the solution of a stochastic differential equation "reflected" at the boundary of a domain $D$, i.e., the reflected diffusions 
- (locally) solves the governing SDE in the interior int $D$,

- stays in $\bar{D}$ almost surely and

- satisfies "some boundary behavior" at the boundary $\partial D$ of the domain.

Let $D \subset \mathbb{R}^{d}$ be an open set. For a given drift vector field $V: \bar{D} \rightarrow \mathbb{R}^{d}$ and diffusion vector fields $V_{1}, \ldots, V_{d}: \bar{D} \rightarrow \mathbb{R}^{d}$, we consider the Skorohod equation

$$
d X_{t}^{x}=V\left(X_{t}\right) d t+\sum_{i=1}^{d} V_{i}\left(X_{t}\right) d B_{t}^{i}+n(t) d Z_{t},
$$

with initial value $X_{0}=x \in D, Z_{0}=0 \in \mathbb{R}$. Here, $n(t)$ is assumed to be an adapted process taking values in the set of inward pointing normal vectors of $\partial D=\bar{D} \backslash D$ at $X_{t}$, provided that $X_{t} \in \partial D$.

Definition 1.1. A pair $(X, Z)=\left(X_{t}, Z_{t}\right)_{t \in[0, \infty[}$ of continuous adapted processes with values in $\bar{D}$ and [0, [, respectively, is a strong solutions of the Skorohod equation [1.1] if $X_{0}=x$, $Z_{0}=0, Z$ is an increasing process satisfying

$$
Z_{t}=\int_{0}^{t} \mathbf{1}_{\partial D}\left(X_{s}\right) d Z_{s}
$$

for all $t \in[0, \infty[$ and the integrated version of equation 1.1] holds true, where $n(t)$ is a given adapted process taking values in the set of inward pointing unit normal vectors of $X_{t} \in \partial D$.

We will often call $X_{t}$ the reflected diffusion and the process $Z_{t}$ its local time.

Remark 1.2. The theory and the numerical analysis of reflected diffusions works just as well in the non-autonomous case, i.e., if the above vector fields $V, V_{1}, \ldots, V_{d}$ are timedependent - sometimes with obvious modifications. For simplicity, we formulate our results only in the autonomous situation.

Remark 1.3. For general domains, the solution of (1.1) depends on the choice of the process $n$. In most situations, however, the boundary of $D$ will be regular enough such that there is almost always a unique inward pointing normal vector. Then $n(t)=n\left(X_{t}\right)$ is a function of the position provided that $X_{t} \in \partial D$.

Remark 1.4. Equation (1.1) defines a reflected diffusion with normal reflection. It is also possible to consider oblique reflections.

Remark 1.5. Equation $(1.2)$ means that the random measure induced by the increasing process $Z$ is concentrated on $\left\{t \mid X_{t} \in \partial D\right\}$. Therefore, we informally call $Z$ local time. Notice, however, that this practice is incorrect because $Z_{t}$ is not the local time in the sense of stochastic analysis. Indeed, let $d=1$ and consider $V \equiv 0$ and $V_{1} \equiv 1$ and the domain $D=] 0, \infty[$. If we start at $x=0$, we have

$$
X_{t}=B_{t}+Z_{t},
$$

noting that the inward pointing normal vector is given by $n \equiv 1$. Given a Brownian motion $W$ and its Lévy transform $\beta_{t}=\int_{0}^{t} \operatorname{sign}\left(W_{s}\right) d W_{s}$, the reflected Brownian motion satisfies

$$
\left|W_{t}\right|=\beta_{t}+L_{t}
$$

by Tanaka's formula, where $L_{t}$ denotes the local time of $W_{t}$ at 0 . Comparing these two equations, we see that $Z_{t}$ is not the local time of $B$, but it is the local time of a Brownian motion $W$ such that

$$
B_{t}=\int_{0}^{t} \operatorname{sign}\left(W_{s}\right) d W_{s},
$$

Moreover, $X_{t}=\left|W_{t}\right|$. Of course, we have the equalities in law

$$
X_{t} \sim\left|B_{t}\right| \text { and } Z_{t} \sim L_{t}(B) \text {. }
$$


Recall that the local time $L_{t}$ of $W$ is defined by

$$
L_{t}=\lim _{\epsilon \rightarrow 0} \frac{1}{2 \epsilon} \int_{0}^{t} \mathbf{1}_{[0, \epsilon]}\left(\left|W_{s}\right|\right) d s,
$$

see, for instance, [17].

For the existence and uniqueness result given by Saisho [18], we have to impose some regularity conditions on the domain. Let $D \subset \mathbb{R}^{d}$ be an open set and consider, for $x \in \partial D=$ $\bar{D} \backslash$ int $D$, the set of inward normal unit vectors $\mathcal{N}_{x}$ defined by

$$
\mathcal{N}_{x}=\bigcup_{r>0} \mathcal{N}_{x, r}, \quad \mathcal{N}_{x, r}=\left\{y \in \mathbb{R}^{d} \mid\|y\|=1, B(x-r y, r) \cap D=\varnothing\right\},
$$

where $B(x, r)$ denotes the sphere with radius $r$ centered at $x$. We impose the following uniform condition on $D$.

Assumption 1.6. There exists a constant $r>0$ such that $\mathcal{N}_{x}=\mathcal{N}_{x, r} \neq \varnothing$ for every $x \in \partial D$. Moreover, we require the vector fields $V, V_{1}, \ldots, V_{d}$ to be bounded and Lipschitz continuous.

Remark 1.7. Note that Assumption 1.6 does not require uniqueness of the inward normal unit vector, in particular, non-differentiable domains are not excluded. In [18], an alternative local assumption is provided. We choose the above uniform condition because it will be satisfied by our numerical examples.

Proposition 1.8. Under the above assumptions, the Skorohod equation (1.1) has a unique strong solution.

Proof. This is [18, Theorem 5.1].

Reflected diffusions give stochastic representations of parabolic PDEs with Neumann boundary conditions. We refer to Freidlin [8] for more details. Let $L$ denote the infinitesimal generator of the SDE 1.1, i.e.,

$$
L f(x)=V_{0} f(x)+\frac{1}{2} \sum_{i=1}^{d} V_{i}^{2} f(x), \quad x \in \mathbb{R}^{d},
$$

where $V_{0}$ is the Stratonovich corrected drift vector field

$$
V_{0}(x)=V(x)-\frac{1}{2} \sum_{i=1}^{d} D V_{i}(x) \cdot V_{i}(x) .
$$

Here, we denote the Jacobian of a vector-field $W: \mathbb{R}^{d} \rightarrow \mathbb{R}^{d}$ by $D W$, and we identify vector fields with first order differential operators via

$$
W f(x)=\nabla f(x) \cdot W(x) .
$$

Note that $W f: \mathbb{R}^{d} \rightarrow \mathbb{R}$ is a function of the same type as $f$, therefore we can apply the vector field $W$ to the function $W f$ as before and denote the result by $W^{2} f$.

We consider a heat equation with Neumann and Dirichlet boundary conditions. Assume that the boundary $\partial D$ of the domain is subdivided into two disjoint sub-domains, $\partial D=$ $\partial D_{R} \cup \partial D_{S}$. We consider the following boundary value problem.

$$
\left\{\begin{aligned}
\frac{\partial}{\partial t} u(t, x)+L u(t, x) & =0, \quad x \in \bar{D}, \\
u(T, x) & =f(x), \quad x \in \bar{D}, \\
u(t, x) & =g(x), \quad x \in \partial D \cap \partial D_{S}, \\
\frac{\partial}{\partial n} u(t, x) & =h(x), \quad x \in \partial D \cap \partial D_{R},
\end{aligned}\right.
$$


where $t \in[0, T], f: \bar{D} \rightarrow \mathbb{R}, g: \bar{D}_{S} \rightarrow \mathbb{R}$ and $h: \partial D_{R} \rightarrow \mathbb{R}$ are sufficiently regular functions, $n(x)$ is assumed to be the unique inward normal vector at $x \in \partial D$ and we denote

$$
\frac{\partial}{\partial n} u(t, x)=\langle\nabla u(t, x), n(x)\rangle
$$

the normal derivative of $u(t, x)$ at $x \in \partial D$. By a solution of 1.3 we understand a function $u \in C^{1,2}([0, T] \times \bar{D})$ satisfying the above PDE.

Remark 1.9. We could additionally let the data, i.e., the driving vector fields and the boundary conditions $g$ and $h$, depend on time. We refrain from doing so for ease of notation.

Proposition 1.10. Assume that the solution u of problem 1.3 has bounded time-derivative, gradient and Hessian matrix for all $(t, x) \in[0, T] \times \bar{D}$. Then we have the stochastic representation

$$
u(t, x)=E\left[f\left(X_{T}\right) \mathbf{1}_{\tau \geq T}+g\left(X_{\tau}\right) \mathbf{1}_{\tau<T}-\int_{t}^{\tau \wedge T} h\left(X_{s}\right) d Z_{s} \mid X_{t}=x\right],
$$

where $\tau=\inf \left\{s \geq t \mid X_{s} \in \partial D_{S}\right\}$ and $x \wedge y:=\min (x, y)$.

Proof. The proposition is a special case of [8, Theorem II.5.1] (which only contains the purely reflecting part). Nevertheless, we repeat the simple and instructive proof in the case of a purely reflecting diffusion. Fixing $T>t>0$ and the stopping time $\tau$ as above. We apply Itô's formula to $u\left(s, X_{s}\right)$ and obtain

$$
\begin{aligned}
u\left(T \wedge \tau, X_{T \wedge \tau}\right)= & u\left(t, X_{t}\right)+\int_{t}^{T \wedge \tau}\left(L u\left(s, X_{s}\right)+\frac{\partial u}{\partial t}\left(s, X_{s}\right)\right) d s \\
& +\sum_{i=1}^{d} \int_{t}^{T \wedge \tau}\left\langle\nabla u\left(s, X_{s}\right), V_{i}\left(X_{s}\right)\right\rangle d B_{s}^{i} \\
& +\int_{t}^{T \wedge \tau}\left\langle\nabla u\left(s, X_{s}\right), n\left(X_{s}\right)\right\rangle d Z_{s} .
\end{aligned}
$$

Note that $d Z_{s}$ is concentrated on $\left\{X_{s} \in \partial D_{R}\right\}$, consequently we may replace the integrand in the last term by $\frac{\partial}{\partial n} u\left(s, X_{s}\right)=h\left(X_{s}\right)$. Furthermore, $L u+\frac{\partial}{\partial t} u=0$ by the PDE. Taking expectations (conditional on $X_{t}=x$ ) concludes the proof since

$$
E\left[u\left(T \wedge \tau, X_{T \wedge \tau}\right) \mid X_{t}=x\right]=E\left[f\left(X_{T}\right) \mathbf{1}_{\tau \geq T}+g\left(X_{\tau}\right) \mathbf{1}_{\tau<T} \mid X_{t}=x\right]
$$

by the boundary conditions.

\section{ApProximation of STOPPED REFLECTED DIFFUSIONS}

We give a review of some approximation methods for a diffusion reflected and stopped at the boundary found in the literature. Again, we concentrate mostly on the reflecting case. We first present the standard projected Euler approach to stopped reflected diffusions, see Costantini, Pacchiarotti and Sartoretto [6]. This approach yields a method with weak order of convergence $1 / 2$ - in particular, they give a simple example, where this convergence rate is precise. Gobet [13] has constructed an algorithm for weak approximation of purely reflecting or purely stopped diffusions based on a half-space approximation of the the boundary of the domain. In certain cases, he can prove a convergence with rate 1. Finally, Bossy, Gobet and Talay [3] have found a method with order one based on symmetrization valid for purely reflecting diffusions, provided that the boundary condition satisfies $h \equiv 0$ (also for oblique reflections). For references to alternative approaches to weak approximation of reflected diffusions we again refer to Gobet [13]. Regarding adaptive approximations of one-dimensional reflected Brownian motions, Asmussen, Glynn and Pitman [2] indicate a simple method using two fixed step sizes chosen according to the distance to the boundary. 
For the stopped diffusion, in the same paper [13], Gobet has introduced a similar halfspace based approach to weak approximation of stopped diffusions. In this case, there is also the adaptive algorithm developed by Dzougoutov et al. [7].

Of course, there are also several papers on strong approximation of stopped and reflected diffusion. As a representative work we cite [19], where the projected Euler method is shown to have strong order of convergence $1 / 4$ for general convex domains and $1 / 2$ for convex polyhedrons in the case of reflected diffusions.

Since the algorithm in [6] is the basis of our algorithms, as well as of the two other algorithms mentioned above, we shall give a rather detailed description. Let us first introduce some general notation: given a partition $0=t_{0}<t_{1}<\cdots<t_{N}=T$, we denote $\Delta t_{i}=t_{i+1}$, $\Delta B_{i}=B_{t_{i+1}}-B_{t_{i}}$ and $\Delta Z_{i}=Z_{t_{i+1}}-Z_{t_{i}}, i=0, \ldots, N-1$. The same convention will also be applied to the approximations to the above quantities, which are denoted by $\bar{X}, \bar{Z}, \ldots$

For simplicity, assume that $\# \mathcal{N}_{x}=1$ for each $x \in \partial D$. Furthermore, assume that we can find a unique projection $\Pi(x) \in \partial D$ for each $x \notin \bar{D}$. Fix $T>0$ and an initial condition $x \in \bar{D}$. Algorithm 2.1 is, in fact, a straight-forward discretization of the Skorohod equation (1.1). Indeed, applying the simplest possible stochastic Taylor expansion (see [15, Chapter 5]) to the reflected diffusion (1.1) on the interval $\left[t_{i}, t_{i+1}\right]$ yields the approximation

$$
X_{t_{i+1}} \approx X_{t_{i}}+V\left(X_{t_{i}}\right) \Delta t_{i}+\sum_{j=1}^{d} V_{j}\left(X_{t_{i}}\right) \Delta B_{i}^{j}+n\left(X_{t_{i}}\right) \Delta Z_{t_{i}} .
$$

Defining

$$
\hat{X}_{i+1}:=X_{t_{i}}+V\left(X_{t_{i}}\right) \Delta t_{i}+\sum_{j=1}^{d} V_{j}\left(X_{t_{i}}\right) \Delta B_{i}^{j},
$$

we get

$$
X_{t_{i+1}}-\hat{X}_{i+1} \approx n\left(X_{t_{i}}\right) \Delta Z_{i+1},
$$

or

$$
\Delta Z_{i+1} \approx\left\|X_{t_{i+1}}-\hat{X}_{i+1}\right\| .
$$

This motivates the following algorithm for weak approximation of a reflected and stopped diffusion.

Algorithm 2.1. Fix a uniform time discretization $0=t_{0}<t_{1}<\cdots<t_{N}=T$, i.e., $\Delta t_{i}=t_{i+1}-t_{i}=\frac{T}{N}, i=0, \ldots, N-1$. Moreover, fix an $i$. $i$. $d$. sequence of random variables $\left(\Delta \bar{B}_{i}\right)_{i=0}^{N-1}$ such that the moments of order up to three of $\Delta \bar{B}_{0}$ (and hence of all $\Delta \bar{B}_{i}$ ) coincide with those of an n-dimensional normal random variable with covariance matrix $\frac{T}{N} \mathrm{id}_{n}$.

(1) $\operatorname{Set} \bar{X}_{0}^{N}=x, \bar{Z}_{0}^{N}=0, N_{\bar{\tau}}=N+1$, and $i=0$.

(2) Compute

$$
\hat{X}_{i+1}^{N}=\bar{X}_{i}^{N}+V\left(\bar{X}_{i}^{N}\right) \Delta t_{i}+\sum_{j=1}^{d} V_{j}\left(\bar{X}_{i}^{N}\right) \Delta \bar{B}_{i}^{j} .
$$

(3) Project to the domain, i.e., set

$$
\begin{aligned}
& \bar{X}_{i+1}^{N}= \begin{cases}\hat{X}_{i+1}^{N}, & \hat{X}_{i+1}^{N} \in \bar{D}, \\
\Pi\left(\hat{X}_{i+1}^{N}\right), & \hat{X}_{i+1}^{N} \notin \bar{D},\end{cases} \\
& \bar{Z}_{i+1}^{N}= \begin{cases}\bar{Z}_{i}^{N}, & \hat{X}_{i+1}^{N} \in \bar{D} \text { or } \bar{X}_{i+1}^{N} \in \partial D_{S}, \\
\bar{Z}_{i}^{N}+\left\|\Pi\left(\hat{X}_{i+1}^{N}\right)-\hat{X}_{i+1}^{N}\right\|, & \text { else. }\end{cases}
\end{aligned}
$$

(4) If $\bar{X}_{i+1}^{N} \in \partial D_{S}$, set $N_{\bar{\tau}}=i+1$ and go to (5). Otherwise, increase $i$ by one. If $i<N$, go back to (2), else go to (5). 
(5) Calculate

$$
\bar{F}^{N}=f\left(\bar{X}_{N}^{N}\right) \mathbf{1}_{N_{\bar{\tau}} \geq N}+g\left(\bar{X}_{N_{\bar{\tau}}}^{N}\right) \mathbf{1}_{N_{\bar{\tau}<N}}-\sum_{i=0}^{N \wedge N_{\bar{\tau}}-1} h\left(\bar{X}_{i+1}^{N}\right) \Delta \bar{Z}_{i}^{N} .
$$

The solution $u(T, x) \approx E\left(\bar{F}^{N}\right)$ is then calculated by Monte Carlo simulation from the distribution $\bar{F}^{N}$ given in Algorithm 2.1 above.

If $u$ is sufficiently regular (e.g., $u \in C_{b}^{3}([0, T] \times \bar{D})$ ) and the random variables $\Delta \bar{B}_{i}$ are either bounded or Gaussian, then we have

$$
\left|u(T, x)-E\left(\bar{F}^{N}\right)\right| \leq \frac{C}{N^{1 / 2}},
$$

where $\Delta \bar{Z}_{i}^{N}=\bar{Z}_{i+1}^{N}-\bar{Z}_{i}^{N}, i=0, \ldots, N-1$. This result is [6, Theorem 3.4, Theorem 3.6], together with a remark in [13].

The basic idea of the half-space approach of Gobet [13] is that the solution of the Skorohod equation (1.1) with constant coefficients can be given explicitly, if $D$ is a half-space. More precisely, assume that we have already constructed $\bar{X}_{l}^{N}$ and $\bar{Z}_{l}^{N}, l=0, \ldots, i$. In order to construct $\bar{X}_{i+1}^{N}$ and $\bar{Z}_{i+1}^{N}$, we project $\bar{X}_{i}^{N}$ to the boundary (along the normal direction), finding the point $\widetilde{X}$. Now we calculate the exact solution $\left(\hat{X}_{i+1}^{N}, \hat{Z}_{i+1}^{N}\right)$ at time $t_{i+1}$ of the reflected diffusion problem with constant coefficients $V\left(\bar{X}_{i}^{N}\right)$ and $V_{1}\left(\bar{X}_{i}^{N}\right), \ldots, V_{n}\left(\bar{X}_{i}^{N}\right)$ and for the domain given by the half-space bounded by the tangent hyperplane on $D$ at $\widetilde{X}$. If the new point $\hat{X}_{i+1}^{N} \in \bar{D}$, then we set $\bar{X}_{i+1}^{N}=\hat{X}_{i+1}^{N}$ and $\bar{Z}_{i+1}^{N}=\bar{Z}_{i}^{N}+\hat{Z}_{i+1}^{N}$. Otherwise, we project $\hat{X}_{i+1}^{N}$ back to $\bar{D}, \bar{X}_{i+1}^{N}=\Pi\left(\hat{X}_{i+1}^{N}\right)$, and add an additional increment to the local time. The quantity of interest $u(T, x)$ is now similarly approximated as in (2.1), with one difference: now the approximation of the local time may increase in a sub-interval $\left[t_{i}, t_{i+1}\right]$, i.e., $\Delta \bar{Z}_{i}^{N}>0$, even though $\bar{X}_{i+1}^{N} \in$ int $D$. Consequently, we need to replace the term $h\left(\bar{X}_{i+1}^{N}\right)$ by $h\left(\Pi\left(\bar{X}_{i+1}^{N}\right)\right)$ this time.

Under some regularity conditions, Gobet [13] proves weak convergence of this method to the solution of the pure Neumann boundary problem $(1.3)$, with $\partial D_{R}=\partial D$. The proven rate of convergence is $\frac{1}{2}$, but it is 1 in case of co-normal reflection, i.e., if the reflection is along the (normalized) direction

$$
\gamma(x)=\left\langle V_{1}(x), n(x)\right\rangle V_{1}(x)+\cdots+\left\langle V_{d}(x), n(x)\right\rangle V_{d}(x) .
$$

Based on numerical evidence, Gobet, however, conjectures that this order of convergence holds for more general situations.

Remark 2.2. Up to our knowledge, there is no explicit construction to the problem of a Brownian motion in a half-space domain $D$ such that the process is reflected on, say, a $(d-1)$-dimensional half-space of $\partial D$ and stopped at the rest of $\partial D$. Therefore, the idea of the Gobet algorithm is difficult to realize when one has both reflection and stopping.

Finally, Bossy, Gobet and Talay [3] have constructed a symmetrized Euler scheme. But for one difference, the scheme is equal to Algorithm 2.1. This difference is the calculation of $\bar{X}_{i+1}^{N}$ from $\hat{X}_{i+1}^{N}$ in the case $\hat{X}_{i+1}^{N} \notin \bar{D}$ : instead of merely projecting $\hat{X}_{i+1}^{N}$ to $\partial D$ as in Algorithm 2.1, the new point $\bar{X}_{i+1}^{N} \in \operatorname{int} D$ is found by projecting onto $\partial D$ and then following the direction $\gamma$ of reflection further into the interior of the domain, such that $\bar{X}_{i+1}^{N}$ and $\hat{X}_{i+1}^{N}$ have the same distance to $\partial D$ along the direction $\gamma$. The algorithm converges with order 1 provided that $h \equiv 0$ even for oblique reflection.

The aforementioned adaptive algorithm for one-dimensional reflected Brownian motions (reflected at 0 ) of [2] works as follows. Given a computer budget $c$, the authors propose to take a step size $h_{c}^{(1)}$ if the current position $\bar{X}<x_{c}$ and $h_{c}^{(2)}$ otherwise. They 
propose to choose

$$
x_{c} \approx c^{-1 / 5}, \quad h_{c}^{(1)} \approx c^{-3 / 5}, \quad h_{c}^{(2)} \approx c^{-2 / 5} .
$$

Thus, the adaptivity only consists in a switch between two fixed step-sizes depending on the current position.

\section{AN ERROR EXPANSION}

Both our algorithms are based on the Algorithm 2.1 of Costantini et al. [6]. In this section, we give an error expansion for this method, which is based on the proof of Proposition 1.10 .

First we explicitly introduce the splitting hinted upon in the proof of Proposition 1.10 namely g $u=u_{S}+u_{R}$, which respectively solve

$$
\left\{\begin{aligned}
\frac{\partial}{\partial t} u_{S}(t, x)+L u_{S}(t, x) & =0, \quad x \in \bar{D}, \\
u_{S}(T, x) & =f(x), \quad x \in \bar{D}, \\
u_{S}(t, x) & =g(x), \quad x \in \partial D \cap \partial D_{S}, \\
\frac{\partial}{\partial n} u_{S}(t, x) & =0, \quad x \in \partial D \cap \partial D_{R},
\end{aligned}\right.
$$

and

$$
\left\{\begin{aligned}
\frac{\partial}{\partial t} u_{R}(t, x)+L u_{R}(t, x) & =0, \quad x \in \bar{D}, \\
u_{R}(T, x) & =0, \quad x \in \bar{D}, \\
u_{R}(t, x) & =0, \quad x \in \partial D \cap \partial D_{S}, \\
\frac{\partial}{\partial n} u_{R}(t, x) & =h(x), \quad x \in \partial D \cap \partial D_{R} .
\end{aligned}\right.
$$

These functions obviously have the stochastic representations

$$
u_{S}(t, x)=E^{t, x}\left[f\left(X_{T}\right) \mathbf{1}_{\tau \geq T}+g\left(X_{\tau}\right) \mathbf{1}_{\tau<T}\right], \quad u_{R}(t, x)=E^{t, x}\left[-\int_{t}^{T \wedge \tau} h\left(X_{s}\right) d Z_{S}\right],
$$

where $E^{t, x}$ denotes the conditional expectation given $X_{t}=x$. For the numerical analysis, we will use the following conventions. Given a grid $0=t_{0}<t_{1}<\cdots<t_{N}=T$, let us denote the discrete Euler process by $\bar{X}_{i}, i=0, \ldots, N$, i.e.,

$$
\bar{X}_{i+1}=\Pi\left(\bar{X}_{i}+V\left(\bar{X}_{i}\right) \Delta t_{i}+\sum_{j=1}^{d} V_{j}\left(\bar{X}_{i}\right) \Delta B_{i}^{j}\right)
$$

with $\bar{X}_{0}=X_{0}$. Here, $\Pi$ denotes the projection operator onto $\bar{D}$. Moreover, we do not restrict ourselves to a deterministic grid in the current discussion. Indeed, $N$ and the stepsizes $\Delta t_{i}=t_{i+1}-t_{i}$ can very well be random, as long as $\Delta t_{i}$ is $\mathcal{F}_{t_{i}}$-measurable for every $i$. (Note that $t_{i}$ is a stopping time in that case.) If $\bar{X}_{i+1} \in \partial D_{R}$, we also define the increment of the approximate local time as

$$
\Delta \bar{Z}_{i}=\left\|\bar{X}_{i}+V\left(\bar{X}_{i}\right) \Delta t_{i}+\sum_{j=1}^{d} V_{j}\left(\bar{X}_{i}\right) \Delta B_{i}^{j}-\bar{X}_{i+1}\right\|,
$$

compare to Algorithm 2.1. Moreover, we set

$$
\bar{\tau}=\inf \left\{t=t_{i} \mid \bar{X}_{i} \in \partial D_{S}\right\}, \quad t_{N_{\bar{\tau}}}=\bar{\tau} \wedge T .
$$


Definition 3.1. The continuous, discretely reflected Euler process $\hat{X}_{t}$ is defined by $\hat{X}_{0}=$ $\hat{X}_{0+}=X_{0}$ and

$$
\hat{X}_{t}=\hat{X}_{t_{i}+}+\int_{t_{i}}^{t} V\left(\hat{X}_{t_{i}+}\right) d s+\sum_{j=1}^{d} \int_{t_{i}}^{t} V_{j}\left(\hat{X}_{t_{i}+}\right) d B_{s}^{j}, \quad t_{i}<t \leq t_{i+1} .
$$

At $t_{i+1}$, we project $\hat{X}$ to the boundary of the domain $D$, but only if the projection is in $\partial D_{R}$ and if the discrete Euler process has not been stopped yet, i.e.,

$$
\hat{X}_{t_{i+1}+}= \begin{cases}\Pi\left(\hat{X}_{t_{i+1}}\right), & \hat{X}_{t_{i+1}} \notin D \text { and } \Pi\left(\hat{X}_{t_{i+1}}\right) \in \partial D_{R} \text { and } t_{i+1} \leq \bar{\tau}, \\ \hat{X}_{t_{i+1}}, & \text { else. }\end{cases}
$$

Moreover, we consider the first hitting time $\hat{\tau}$ of $\hat{X}$ at the set $\partial D_{S}$ and set

$$
N_{\hat{\tau}}=\inf \left\{0 \leq i \leq N \mid t_{i+1}>\hat{\tau}\right\} \wedge N .
$$

Remark 3.2. The word "continuous" in the name of $\hat{X}$ only refers to the time variable. In fact, $\hat{X}$ is piecewise continuous.

Remark 3.3. Different to ordinary and to the purely stopped diffusion in [7]), the process $\hat{X}$ defined above cannot be regarded as a simple interpolation of the discrete process $\bar{X}$. Indeed, notice that both $\hat{\tau}<\bar{\tau}$ and $\bar{\tau}<\hat{\tau}$ are possible cases. The second happens, for instance, if $\bar{X}_{i}=\hat{X}_{t_{i}}, \hat{X}_{t}$ leaves $D$ through $\partial D_{R}$ and stays outside $D$ until $t_{i+1}$ such that $\Pi\left(\hat{X}_{t_{i+1}}\right) \in \partial D_{S}$. In this case, $\bar{X}_{i+1} \in \partial D_{S}$ and $\bar{\tau}=t_{i+1}$, whereas $\hat{\tau}>t_{i+1}$ and $\hat{X}_{t_{i+1}+} \neq \bar{X}_{i+1}$. For a sketch of $\hat{\tau}>\bar{\tau}$ see Figure 1 .

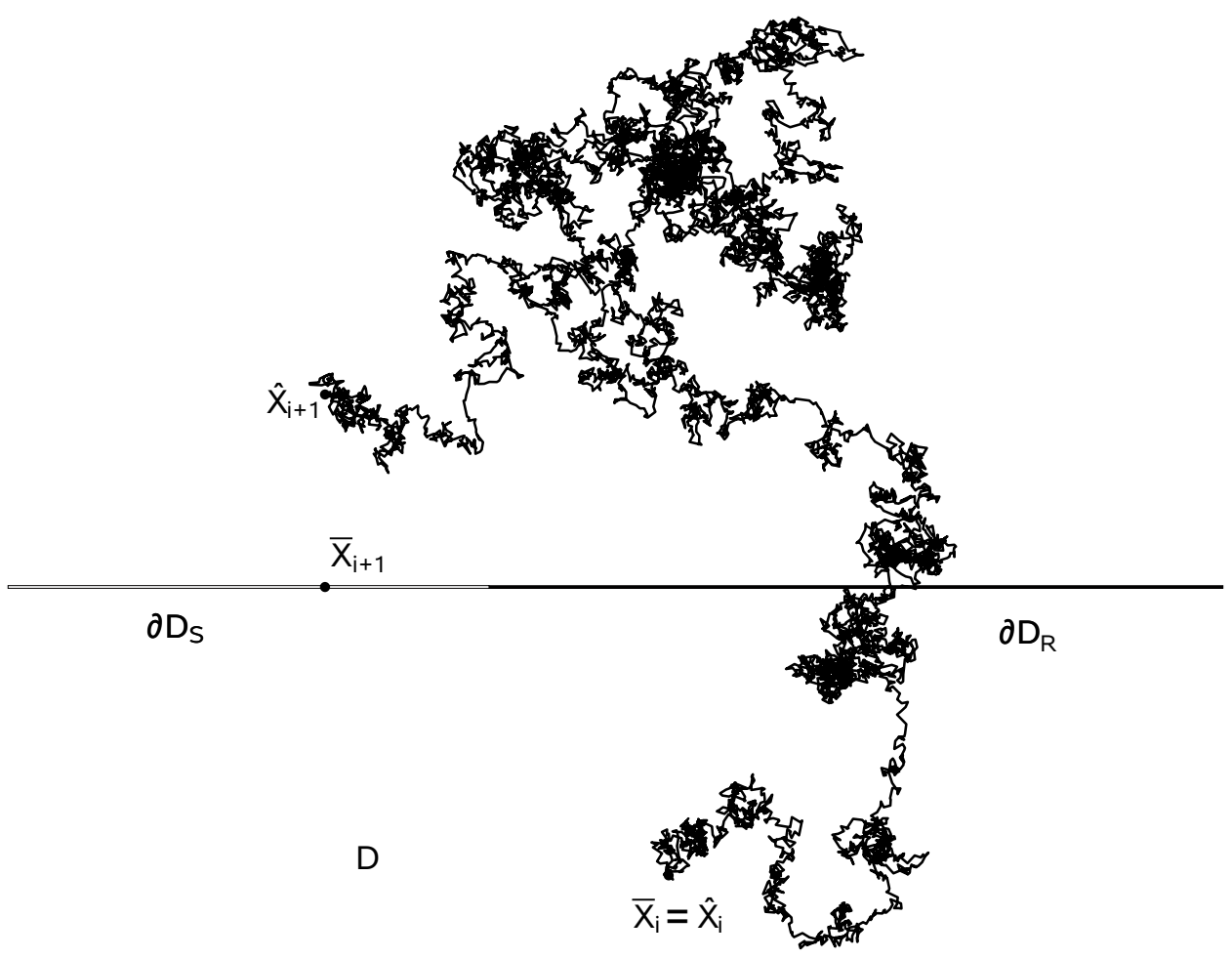

Figure 1. Example for $\hat{\tau}>\bar{\tau}$ as described in Remark 3.3 .

For the considerations in the following propositions, let us assume that the solution $u$ of 1.3 can be extended to $[0, T] \times \mathbb{R}^{d}$ in the sense that there is a function $v:[0, T] \times \mathbb{R}^{d} \rightarrow \mathbb{R}$ 
that coincides with $u$ on $[0, T] \times \bar{D}$ and solves $\frac{\partial}{\partial t} v+L v=0$ globally and is $C^{1,2}$ globally. We will denote the extension again with the letter $u$. With both Dirichlet and Neumann conditions present, this assumption is hard to verify.

Remark 3.4. To illustrate that the above assumption can be satisfied for some class of problems, consider the following situation. Assume that the driving vector fields $V, V_{1}, \ldots, V_{d}$ are real-analytic and can be extended (as analytic functions) to some neighborhood $U \supset D$. Moreover, assume that we are given an analytic solution $u:[0, T] \times \bar{D} \rightarrow \mathbb{R}$ of $(1.3)$ which can be extended as an analytic function to $U$. Then the extended function $u:[0, T] \times U \rightarrow \mathbb{R}$ satisfies the PDE

$$
\frac{\partial}{\partial t} u(t, x)+L u(t, x)=0, \quad x \in U
$$

since $\frac{\partial}{\partial t} u(t, x)+L u(t, x)$ is an analytic function which is zero on $D \subset U$.

Proposition 3.5. The weak error of the Euler scheme (3.4) for approximation of the solution of 1.3 by

$$
\bar{u}\left(0, x_{0}\right)=E\left[f\left(\bar{X}_{N \wedge N_{\bar{\tau}}}\right)-\sum_{i=0}^{N_{\bar{\tau}}-1} h\left(\bar{X}_{i+1}\right) \Delta \bar{Z}_{i}\right]
$$

satisfies the error expansion

$$
\begin{aligned}
& \bar{u}\left(0, x_{0}\right)-u\left(0, x_{0}\right)=-\frac{1}{2} E\left[\sum_{i=0}^{N_{\bar{\tau}}-1-\mathbf{1}_{\{\bar{\tau}<T\}}} u_{R, n n}\left(t_{i+1}, \bar{X}_{i+1}, \Delta \bar{Z}_{i}\right)\left(\Delta \bar{Z}_{i}\right)^{2}\right]- \\
& -\frac{1}{2} E\left[\sum_{i=0}^{N_{\bar{\tau}} \wedge N_{\hat{\tau}}-1} u_{S, n n}\left(t_{i+1}, \bar{X}_{i+1}\right)\left(\Delta \bar{Z}_{i}\right)^{2}\right]+ \\
& +E\left[\int_{0}^{\bar{\tau} \wedge T}(\bar{L}-L) u_{R}\left(s, \hat{X}_{s}\right) d s\right]+E\left[\int_{0}^{\hat{\tau} \wedge T}(\bar{L}-L) u_{S}\left(s, \hat{X}_{s}\right) d s\right]+ \\
& +E\left[\left(\partial_{t} u_{R}\left(\bar{\tau}, \bar{X}_{N_{\bar{\tau}}}\right) \Delta t_{N_{\bar{\tau}}-1}+\partial_{n} u_{R}\left(\bar{\tau}, \bar{X}_{N_{\bar{\tau}}}\right)\left\langle n\left(\bar{X}_{N_{\bar{\tau}}}\right), \bar{X}_{N_{\bar{\tau}}}-\bar{X}_{N_{\bar{\tau}}-1}\right\rangle\right) \mathbf{1}_{\{\bar{\tau}<T\}}\right]+ \\
& +E\left[\sum_{i=0}^{N-1}\left(g\left(\bar{X}_{N_{\bar{\tau}}}\right) \mathbf{1}_{N_{\bar{\tau}}<N}+f\left(\bar{X}_{N_{\bar{\tau}}}\right) \mathbf{1}_{N_{\bar{\tau}}=N}-g\left(\Pi_{S}\left(\frac{\hat{X}_{t_{i}+}+\hat{X}_{t_{i+1}}}{2}\right)\right)\right) P\left(t_{i} \leq \hat{\tau}<t_{i+1} \mid X\right)\right]+ \\
& + \text { terms of higher order, }
\end{aligned}
$$

where $u_{R, n n}$ and $u_{S, n n}$ are defined in (3.9).

Proof. We analyze the error of the discrete Euler scheme (3.4) by splitting it into the error contribution for the purely stopped part $u_{S}\left(0, x_{0}\right)$ and for $u_{R}\left(0, x_{0}\right)$, respectively. In other 
words, using $u(t, x)=u_{R}(t, x)+u_{S}(t, x)$, we have

$$
\begin{aligned}
& \bar{u}\left(0, x_{0}\right)-u\left(0, x_{0}\right)=E\left[u\left(T \wedge \bar{\tau}, \bar{X}_{N \wedge N_{\bar{\tau}}}\right)-u\left(0, x_{0}\right)-\sum_{i=0}^{N \wedge N_{\bar{\tau}}-1} h\left(\bar{X}_{i+1}\right) \Delta \bar{Z}_{i}\right] \\
& =E\left[\sum_{i=0}^{N \wedge N_{\bar{\tau}}-1}\left(\left[u_{R}\left(t_{i+1}, \bar{X}_{i+1}\right)-u_{R}\left(t_{i}, \bar{X}_{i}\right)\right]-h\left(\bar{X}_{i}\right) \Delta \bar{Z}_{i}\right)+\right. \\
& \left.+u_{S}\left(T \wedge \bar{\tau}, \bar{X}_{N \wedge N_{\bar{\tau}}}\right)-u_{S}\left(0, x_{0}\right)\right] \\
& =E\left[\sum_{i=0}^{N \wedge N_{\bar{\tau}}-1-\mathbf{1}_{N_{\bar{\tau}}<N}} u_{R}\left(t_{i+1}, \bar{X}_{i+1}\right)-u_{R}\left(t_{i+1}, \hat{X}_{i+1}\right)-h\left(\bar{X}_{i+1}\right) \Delta \bar{Z}_{i}\right]+ \\
& +E\left[\left(u_{R}\left(t_{N_{\bar{\tau}}}, \bar{X}_{i+1}\right)-u_{R}\left(t_{i+1}, \hat{X}_{i+1}\right)-h\left(\bar{X}_{i+1}\right) \Delta \bar{Z}_{i}\right) \mathbf{1}_{N_{\bar{\tau}} \leq N}\right]+ \\
& +E\left[\sum_{i=0}^{N \wedge N_{\bar{\tau}}-1} u_{R}\left(t_{i+1}, \hat{X}_{i+1}\right)-u_{R}\left(t_{i}, \bar{X}_{i}\right)\right]+ \\
& +E\left[u_{S}\left(T \wedge \bar{\tau}, \bar{X}_{N \wedge N_{\bar{\tau}}}\right)-u_{S}\left(0, x_{0}\right)\right] \\
& =(I)+(I I)+(I I I)+(I V) .
\end{aligned}
$$

Let us first consider 3.7c). By Ito's formula and the fact that $\bar{X}_{i}=\hat{X}_{t_{i}+}$ for $i<N_{\bar{\tau}}$,

$$
\begin{aligned}
u_{R}\left(t_{i+1}, \hat{X}_{i+1}\right)-u_{R}\left(t_{i}, \bar{X}_{i}\right) & =\int_{t_{i}}^{t_{i+1}}\left(\frac{\partial u_{R}}{\partial t}\left(t, \hat{X}_{t}\right)+\bar{L}_{\bar{X}_{i}} u_{R}\left(t, \hat{X}_{t}\right)\right) d t+\cdots \\
& =\int_{t_{i}}^{t_{i+1}}\left(\bar{L}_{\bar{X}_{i}}-L\right) u_{R}\left(t, \hat{X}_{t}\right) d t+\cdots
\end{aligned}
$$

where "..." denotes a martingale term and $\bar{L}_{y}$ denotes the infinitesimal generator of the SDE driven by the constant vector fields $V(y), V_{1}(y), \ldots, V_{d}(y) \in \mathbb{R}^{d}$. Consequently, the term 3.7c is equal to

$$
(I I I)=E\left[\int_{0}^{\bar{\tau}}(\bar{L}-L) u_{r}\left(t, \hat{X}_{t}\right) d t\right] .
$$

Classical Taylor expansion allows us to re-express the first term in the error expansion 3.7. Indeed,

for $0 \leq i \leq N_{\bar{\tau}-2}$ implies that

$$
\bar{X}_{i+1}=\hat{X}_{i+1}+n\left(\bar{X}_{i+1}\right) \Delta \bar{Z}_{i}
$$

$$
\begin{aligned}
u_{R}\left(t_{i+1}, \bar{X}_{i+1}\right)-u_{R}\left(t_{i+1}, \hat{X}_{i+1}\right) & =u_{R}\left(t_{i+1}, \bar{X}_{i+1}\right)-u_{R}\left(t_{i+1}, \bar{X}_{i+1}-n\left(\bar{X}_{i+1}\right) \Delta \bar{Z}_{i}\right) \\
& =\Delta \bar{Z}_{i} \frac{\partial}{\partial n} u_{R}\left(t_{i+1}, \bar{X}_{i+1}\right)-\left(\Delta \bar{Z}_{i}\right)^{2} u_{R, n n}\left(t_{i+1}, \bar{X}_{i+1}, \Delta \bar{Z}_{i}\right),
\end{aligned}
$$

where

$$
u_{R, n n}(t, x, z)=\int_{0}^{1}(1-\theta) \frac{\partial^{2}}{\partial n^{2}} u_{R}(t, x-\theta z n(x)) d \theta
$$

is a Taylor remainder term. Consequently, we get

$$
(I)=E\left[\sum_{i=0}^{N \wedge N_{\bar{\tau}}-1-\mathbf{1}_{N_{\bar{\tau}}<N}}\left(\Delta \bar{Z}_{i}\right)^{2} u_{R, n n}\left(t_{i+1}, \bar{X}_{i+1}, \Delta \bar{Z}_{i}\right)\right] .
$$

Note that the above computation does not work for the sub-interval directly before hitting the stopping boundary, since then $\hat{X}_{i+1}$ is projected onto the stopping boundary, and the corresponding directional derivative is not given by the equation (unlike $h$, the directional 
derivative in the projection direction over the reflecting boundary). We can, however, do make an ordinary Taylor expansion

$$
\begin{aligned}
& E\left[\left(u_{R}\left(t_{N_{\bar{\tau}}}, \bar{X}_{N_{\bar{\tau}}}\right)-u_{R}\left(t_{N_{\bar{\tau}}-1}, \bar{X}_{N_{\bar{\tau}}-1}\right)\right) \mathbf{1}_{\bar{\tau} \leq N}\right]= \\
& \left.E\left[\left(\partial_{t} u_{R}\left(t_{N_{\bar{\tau}}}, \bar{X}_{N_{\bar{\tau}}}\right) \Delta t_{N_{\bar{\tau}}-1}+\left\langle\nabla u_{R}\left(t_{N_{\bar{\tau}}}, \bar{X}_{N_{\bar{\tau}}}\right)\right), \bar{X}_{N_{\bar{\tau}}}-\bar{X}_{N_{\bar{\tau}}-1}\right\rangle\right) \mathbf{1}_{\bar{\tau} \leq N}\right]+O(\ldots) .
\end{aligned}
$$

While this expression is still not computable, we expect it to give a small contribution of order $O\left(\sqrt{\Delta t_{N_{\bar{\tau}}-1}}\right)$ only. We represent $\bar{X}_{N_{\bar{\tau}}}-\bar{X}_{N_{\bar{\tau}}-1}$ as the sum of its component in direction $n\left(\bar{X}_{N_{\bar{\tau}}}\right)$ and the complement. Since $n$ is a normal vector to $\partial D$, the complement is tangential to $\partial D$ - as long as $\bar{X}_{N_{\bar{\tau}}}$ is not a corner or an otherwise non-smooth point of $\partial D$. Since $\left.u_{R}\right|_{\partial D_{S}} \equiv 0$, we know that any directional derivative of $u_{R}$ at $\partial D_{S}$ is zero. Consequently, we obtain

$$
(I I)=E\left[\left(\partial_{t} u_{R}\left(t_{N_{\bar{\tau}}}, \bar{X}_{N_{\bar{\tau}}}\right) \Delta t_{N_{\bar{\tau}}-1}+\partial_{n} u_{R}\left(t_{N_{\bar{\tau}}}, \bar{X}_{N_{\bar{\tau}}}\right)\left\langle n\left(\bar{X}_{N_{\bar{\tau}}}\right), \bar{X}_{N_{\bar{\tau}}}-\bar{X}_{N_{\bar{\tau}}-1}\right\rangle\right) \mathbf{1}_{\{\bar{\tau}<T\}}\right]+O(\ldots) .
$$

We are left with the error from the pure stopping problem. As usual, we are aiming at Ito's formula using $\hat{X}$. Therefore, we subdivide the contribution into

$$
\begin{aligned}
(I V)= & E\left[u_{S}\left(\bar{\tau} \wedge T, \bar{X}_{N_{\bar{\tau}} \wedge N}\right)-u_{S}\left(\hat{\tau} \wedge T, \hat{X}_{\hat{\tau} \wedge T}\right)\right]+ \\
& +E\left[u_{S}\left(\hat{\tau} \wedge T, \hat{X}_{\hat{\tau} \wedge T}\right)-u_{S}\left(0, x_{0}\right)\right] \\
= & (V)+(V I) .
\end{aligned}
$$

Noting that $\bar{\tau}<\hat{\tau}$ is very well possible, 3.12b can be further expanded into

$$
\begin{aligned}
(V I)=E\left[u_{S}\left(\hat{\tau} \wedge T, \hat{X}_{\hat{\tau} \wedge T}\right)-u_{S}\left(\hat{\tau} \wedge \bar{\tau} \wedge T, \hat{X}_{\hat{\tau} \wedge \bar{\tau} \wedge T}\right)\right]+ & \\
& +E\left[u_{S}\left(\hat{\tau} \wedge \bar{\tau} \wedge T, \hat{X}_{\hat{\tau} \wedge \bar{\tau} \wedge T}\right)-u_{S}\left(0, x_{0}\right)\right] .
\end{aligned}
$$

Here, $\hat{X}$ interpolates the discrete Euler process $\bar{X}$ until $\bar{\tau}$. Therefore, the second term of the above expression can be expanded using Ito's formula between the mesh points and Taylor's formula at the mesh points where reflection occurs, giving us

$$
\begin{aligned}
E\left[u_{S}\left(\hat{\tau} \wedge \bar{\tau} \wedge T, \hat{X}_{\hat{\tau} \wedge \bar{\tau} \wedge T}\right)-u_{S}\left(0, x_{0}\right)\right]=E\left[\sum_{i=0}^{N_{\bar{\tau}} \wedge N_{\hat{\tau}}-1} \int_{t_{i}}^{t_{i+1}}(\bar{L}-L) u_{S}\left(s, \hat{X}_{S}\right) d s-\right. & \\
& \left.-\sum_{i=0}^{N_{\bar{\tau}} \wedge N_{\hat{\tau}}-1} \frac{1}{2} u_{S, n n}\left(t_{i+1}, \bar{X}_{i+1}, \Delta \bar{Z}_{i}\right)\left(\Delta \bar{Z}_{i}\right)^{2}\right],
\end{aligned}
$$

where we used $\partial_{n} u_{S}(t, x)=0, x \in \partial D_{R}$. On the other hand, after $\bar{\tau} \wedge \hat{\tau}, \hat{X}$ is either a continuous process or a killed process, so we directly get

$$
E\left[u_{S}\left(\hat{\tau} \wedge T, \hat{X}_{\hat{\tau} \wedge T}\right)-u_{S}\left(\hat{\tau} \wedge \bar{\tau} \wedge T, \hat{X}_{\hat{\tau} \wedge \bar{\tau} \wedge T}\right)\right]=E\left[\int_{\bar{\tau} \wedge \hat{\tau} \wedge T}^{\hat{\tau} \wedge T}(\bar{L}-L) u_{S}\left(s, \hat{X}_{s}\right) d s\right] .
$$

Consequently, $3.12 \mathrm{~b}$ is given by

$$
(V I)=\left[\int_{0}^{T \wedge \hat{\tau}}(\bar{L}-L) u_{S}\left(t, \hat{X}_{t}\right) d t-\sum_{i=0}^{N_{\bar{\tau}} \wedge N_{\hat{\tau}} \wedge N-1} \frac{1}{2} u_{S, n n}\left(t_{i+1}, \bar{X}_{i+1}, \Delta \bar{Z}_{i}\right)\left(\Delta \bar{Z}_{i}\right)^{2}\right] .
$$

This leaves us only with the term $3.12 \mathrm{a}$. Here we apply the reasoning from [7], but once again we have to observe that $\hat{\tau}>\bar{\tau}$ is very well possible due to reflections. Indeed, let us denote by $\mathcal{X}$ the $\sigma$-algebra generated by all the simulated quantities, i.e.,

$$
\mathcal{X}=\sigma\left(\left\{\hat{X}_{t_{i}} \mid 0 \leq i \leq N\right\}\right)
$$

Then we have $P\left(t_{i} \leq \hat{\tau} \leq t_{i+1} \mid \mathcal{X}\right)>0$ for all $i$ - unless there is an $i$ with $\hat{X}_{t_{i}} \in \partial D_{S}$, an event with probability zero. After conditioning on $\mathcal{X}$, we furthermore condition on $\left\{\hat{\tau} \in\left[t_{i}, t_{i+1}[\}\right.\right.$ 
and approximate

$$
E\left[u_{S}\left(\hat{\tau} \wedge T, \hat{X}_{\hat{\tau} \wedge T}\right) \mid X \vee \sigma\left(\left\{\hat{\tau} \in\left[t_{i}, t_{i+1}[\}\right)\right] \approx g\left(\Pi_{S}\left(\frac{\hat{X}_{t_{i}+}+\hat{X}_{t_{i+1}}}{2}\right)\right),\right.\right.
$$

where $\Pi_{S}$ denotes the projection to the stopping boundary $\partial D_{S}$. This gives us

$$
(V) \approx E\left[\sum_{i=0}^{N-1}\left(g\left(\bar{X}_{N_{\bar{\tau}}}\right) \mathbf{1}_{N_{\bar{\tau}}<N}+f\left(\bar{X}_{N_{\bar{\tau}}}\right) \mathbf{1}_{N_{\bar{\tau}}=N}-g\left(\Pi_{S}\left(\frac{\hat{X}_{t_{i}+}+\hat{X}_{t_{i+1}}}{2}\right)\right)\right) P\left(t_{i} \leq \hat{\tau}<t_{i+1} \mid X\right)\right] .
$$

Note that we have to sum over the whole range $i \in\{0, \ldots, N-1\}$, since $\hat{\tau}$ is not directly observable from the simulation and can take arbitrarily large values.

Remark 3.6. In order to compute an error estimate based on Proposition 3.5, we need to approximate $\partial_{t} u_{R}$ and $\partial_{n} u_{R}$ on $\partial D_{S}$ and $\partial_{n}^{2} u_{R}, \partial_{n}^{2} u_{S}$ on $\partial D_{R}$. Moreover, we ignore all terms involving $\hat{\tau}$. Since these approximations may lead to undesired cancellation effects, if the sign of the different contribution is not found correctly, it might be advisable to compute an upper bound by taking absolute values of all the error contributions. A minor possible modification of 3.14 is to replace $g\left(\Pi_{S}\left(\frac{\hat{X}_{t^{+}}+\hat{X}_{t_{i+1}}}{2}\right)\right)$ by $\frac{g\left(\hat{X}_{i^{+}}\right)+g\left(\hat{X}_{t_{i+1}}\right)}{2}$ in the last line.

Remark 3.7. For an estimate of $P\left(t_{i} \leq \hat{\tau}<t_{i+1} \mid X\right)$ see Appendix A.

Remark 3.8. Note that the absolute value of the leading order term in the error expansion can be bounded by

$$
\begin{aligned}
& \text { Error } \leq E\left[\sum_{i=0}^{N-1}\left|u_{n n}\left(t_{i+1}, \bar{X}_{i+1}\right)\right|\left(\Delta \bar{Z}_{i}\right)^{2}\right]+ \\
& +E\left[\left(\partial_{t} u_{R}\left(\bar{\tau}, \bar{X}_{N_{\bar{\tau}}}\right) \Delta t_{N_{\bar{\tau}}-1}+\partial_{n} u_{R}\left(\bar{\tau}, \bar{X}_{N_{\bar{\tau}}}\right)\left\langle n\left(\bar{X}_{N_{\bar{\tau}}}\right), \bar{X}_{N_{\bar{\tau}}}-\bar{X}_{N_{\bar{\tau}}-1}\right\rangle\right) \mathbf{1}_{\{\bar{\tau}<T\}}\right]+ \\
& +E\left[\sum_{i=0}^{N-1}\left(g\left(\bar{X}_{N_{\bar{\tau}}}\right) \mathbf{1}_{N_{\bar{\tau}}<N}+f\left(\bar{X}_{N_{\bar{\tau}}} \mathbf{1}_{N_{\bar{\tau}}=N}-g\left(\Pi_{S}\left(\frac{\hat{X}_{t_{i}+}+\hat{X}_{t_{i+1}}}{2}\right)\right)\right) P\left(t_{i} \leq \hat{\tau}<t_{i+1} \mid \mathcal{X}\right)\right]\right.
\end{aligned}
$$

up to terms of higher order. Therefore, we may interpret the leading error term as a sum of three terms: the first term can be naturally interpreted as the error of a purely reflecting problem; the last term corresponds to the error term of a purely stopped diffusion, whereas the middle term is peculiar to the combination of a stopping and a reflecting boundary. Note, however, that the last component of the above sum differs from the error term of a stopped diffusion in the sense that $\hat{\tau}$ is only triggered if $\partial D_{S}$ is hit. Therefore, the corresponding stopped diffusion is stopped only at $\partial D_{S}$ and may even wander outside $D$.

Corollary 3.9. In the case of a pure reflecting diffusion, i.e., $\partial D_{R}=\partial D$, the error representation is given by

$$
\bar{u}\left(0, x_{0}\right)-u\left(0, x_{0}\right)=\int_{0}^{T} E\left((\bar{L}-L) u\left(t, \hat{X}_{t}\right)\right) d t-E\left[\sum_{i=0}^{N-1}\left(\Delta \bar{Z}_{i}\right)^{2} u_{n n}\left(t_{i+1}, \bar{X}_{i+1}, \Delta \bar{Z}_{i}\right)\right] .
$$

Remark 3.10. The error expansion in Corollary 3.9 naturally splits into two parts. The first part, i.e.,

$$
\int_{0}^{T} E\left[(\bar{L}-L) u\left(t, \bar{X}_{t}^{N}\right)\right] d t
$$

is well understood since this is the first order term for the usual Euler-Maruyama method for (non-reflected) SDEs. We will refer to this part as interior error, since its main contributions are discretization errors in the interior of the domain. Much more interesting for our study is the second term in the error expansion,

$$
E\left[\sum_{i=0}^{N-1}\left(\Delta \bar{Z}_{i}^{N}\right)^{2} \int_{0}^{1}(1-\theta) \frac{\partial^{2}}{\partial n\left(\bar{X}_{i+1}^{N}\right)^{2}} u\left(t_{i+1}, \bar{X}_{i+1}^{N}-\theta \Delta \bar{Z}_{i}^{N} n\left(\bar{X}_{i+1}^{N}\right)\right) d \theta\right],
$$


which measures the contribution from the reflection. In order to assert the meaning of 3.16, let us take a first look at it. Assume that the equation is nice enough such that $\frac{\partial^{2}}{\partial n^{2}} u$ is uniformly bounded on $[0, T] \times \bar{D}$. Then the integral term can be bounded by some constant, and we are left with

$$
\text { const } E\left[\sum_{i=0}^{N-1}\left(\Delta \bar{Z}_{i}^{N}\right)^{2}\right] \text {. }
$$

Heuristically, the number of hits at the boundary, i.e., the number of indices $i$ such that $\Delta \bar{Z}_{i}^{N} \neq 0$, increases like $\sqrt{N}$ with the number of time-steps. On the other hand, given a hit takes place, then $\Delta \bar{Z}_{i}^{N} \approx \sqrt{\Delta t_{i}}$, since $\Delta \bar{Z}_{i}^{N}$ is, asymptotically, the modulus of the increment of a Brownian motion, or the increment of a Brownian motion conditioned to be positive. Combining these heuristic observations, we get

$$
\text { const } E\left[\sum_{i=0}^{N-1}\left(\Delta \bar{Z}_{i}^{N}\right)^{2}\right] \approx C \sqrt{N} \times \frac{1}{N} \approx \frac{C}{\sqrt{N}},
$$

as it should be by the results of [6].

The above heuristics can be partly backed up by the following observation.

Lemma 3.11. Given a one-dimensional Brownian motion $\left(B_{t}\right)_{t \in[0, \infty[}$ and a fixed time $T>$ 0 . For $N \in \mathbb{N}$ let $\Delta t=T / N$ and $t_{n}=n \Delta t, n=0, \ldots, N$. By abuse of notation, we define

$$
\Delta Z_{n}=\left(\left|B_{t_{n}}\right|+\operatorname{sign}\left(B_{t_{n}}\right) \Delta B_{n}\right)_{-}
$$

where, as usual, $\Delta B_{n}=B_{t_{n+1}}-B_{t_{n}}$ and $n \in\{0, \ldots, N-1\}$. Then we have

$$
E\left[\sum_{n=0}^{N-1} \mathbf{1}_{] 0, \infty[}\left(\Delta Z_{n}\right)\right] \leq \frac{2}{\pi} \sqrt{N}+C,
$$

where $C \approx 0.07127$ is a numerical constant.

Remark 3.12. $\left|B_{t}\right|$ is the continuously reflected Brownian motion at 0 . Therefore, Lemma 3.11 establishes the desired asymptotics $E(\widetilde{N}) \leq$ const $\sqrt{N}$ for the number of hits at the boundary $\widetilde{N}$, if we assume that $\bar{X}_{\left\lfloor t_{n}\right\rfloor}^{N}$ has already converged to its weak limit $\left|B_{t_{n}}\right|$.

Proof of Lemma 3.11. By independence of $B_{t_{n}}$ and $\Delta B_{n}$, we get

$$
\begin{aligned}
P\left(\Delta Z_{n}>0\right) & =P\left(\left|B_{t_{n}}\right|+\operatorname{sign}\left(B_{t_{n}}\right) \Delta B_{n}<0\right) \\
& =P\left(\sqrt{t_{n}}\left|Y_{1}\right|+\sqrt{\Delta t} Y_{2}<0\right) \\
& =P\left(\left|Y_{1}\right|<\frac{1}{\sqrt{n}} Y_{2}\right)
\end{aligned}
$$

for two independent standard normal random variables $Y_{1}$ and $Y_{2}, n \geq 1$. This means that we need to compute the probability of the convex cone

$$
A=\left\{\begin{array}{l|l}
\left(y_{1}, y_{2}\right) \in \mathbb{R}^{2} \mid & \left.y_{1} \mid<\frac{1}{\sqrt{n}} y_{2}\right\}
\end{array}\right.
$$

with respect to the standard, two-dimensional Gaussian measure. By the rotation-invariance of the Gaussian measure, the probability of the cone $A$ coincides with the area of $A \cap D^{1}$ divided by the total area of $D^{1}$, where $D^{1}$ denotes the unit sphere, symbolically,

$$
P(Y \in A)=\frac{\lambda^{2}\left(A \cap D^{1}\right)}{\lambda^{2}\left(D^{1}\right)}=\frac{\lambda^{2}\left(A \cap D^{1}\right)}{\pi} .
$$


Let $\bar{A}=A \cup(-A)=\left\{\left(y_{1}, y_{2}\right) \in \mathbb{R}^{2} \mid y_{1}^{2}<\frac{1}{n} y_{2}^{2}\right\}$. In polar coordinates, we may rewrite $\bar{A} \equiv\left\{(r, \phi) \mid \phi \in\left[0,2 \pi\left[, \tan ^{2} \phi>n\right\}\right.\right.$

$=[0, \infty[\times(] \arctan (\sqrt{n}), \pi-\arctan (\sqrt{n})[\cup] \pi+\arctan (\sqrt{n}), 2 \pi-\arctan (\sqrt{n})[)$,

where we choose arctan to take values in ] $-\pi / 2, \pi / 2[$. Consequently, we get

$$
\lambda^{2}\left(\bar{A} \cap D^{1}\right)=\pi-2 \arctan (\sqrt{n}),
$$

implying

$$
P\left(\Delta Z_{n}>0\right)=\frac{\pi-2 \arctan (\sqrt{n})}{2 \pi} .
$$

The Laurent series of the $\arctan$ for $|x|>1$ is given by

$$
\arctan (x)=\frac{\pi}{2}-\frac{1}{x}+\frac{1}{3 x^{3}}-\frac{1}{5 x^{5}}+\cdots,
$$

see Abramowitz and Stegun [1]. This implies

$$
\arctan (x) \geq \frac{\pi}{2}-\frac{1}{x}, \quad x \geq 1
$$

Equation 3.18 can now be bounded by

$$
P\left(\Delta Z_{n}>0\right) \leq \frac{1}{\pi \sqrt{n}}, \quad n>0,
$$

implying that

$$
\sum_{n=0}^{N-1} P\left(\Delta Z_{n}>0\right) \leq \frac{1}{2}+\sum_{n=1}^{N-1} \frac{1}{\pi \sqrt{n}}
$$

The series expansion

$$
\sqrt{1+\frac{1}{x}}=\frac{2}{3} \sum_{i=0}^{\infty} \frac{1}{B\left(\frac{3}{2}-i, i+1\right) x^{i}}
$$

valid for $|x|$ large enough, implies

$$
g(x):=x\left(\frac{1}{2}-\sqrt{x}(\sqrt{x+1}-\sqrt{x})\right)=\frac{1}{8}-\frac{2}{3} \sum_{i=3}^{\infty} \frac{1}{B\left(\frac{3}{2}-i, i+1\right) x^{i-2}},
$$

where $B$ denotes the Beta-function. This shows that $\lim _{x \rightarrow \infty} g(x)=\frac{1}{8}$. Moreover,

$$
g^{\prime}(x)=\frac{\sqrt{x+1} \sqrt{x}-4 x^{2}-3 x+4 x^{3 / 2} \sqrt{x+1}}{2 \sqrt{x} \sqrt{x+1}}>0, \quad x>0 .
$$

Consequently, $g(x)<\frac{1}{8}$ for $x>0$, and, for $x=n$, we get

$$
\frac{1}{\pi \sqrt{n}} \leq \frac{2}{\pi}\left((\sqrt{n+1}-\sqrt{n})+\frac{1}{8 n^{3 / 2}}\right) .
$$

Putting everything together, we obtain

$$
\begin{aligned}
\sum_{n=0}^{N-1} P\left(\Delta Z_{n}>0\right) & \leq \frac{1}{2}+\frac{2}{\pi} \sum_{n=1}^{N-1}(\sqrt{n+1}-\sqrt{n})+\frac{1}{4 \pi} \sum_{n=1}^{N-1} \frac{1}{n^{3 / 2}} \\
& \leq \frac{1}{2}+\frac{2}{\pi}(\sqrt{N}-1)+\frac{1}{4 \pi} \zeta(3 / 2) .
\end{aligned}
$$


In the remainder of this article, we present two new algorithms based on the error expansion in Proposition 3.5. where we mainly concentrate on the reflecting part. The first algorithm is an adaptive algorithm. Numerical experiments (with some theoretical underpinning, see Proposition 4.15 indicate a weak convergence with a rate between $1 / 2$ and 1 , probably around 0.8 . In Section 4 we present the adaptive algorithm for purely reflected diffusions, which we combine, in light of Proposition 3.5, with the existing adaptive algorithm for stopped diffusions of Dzougoutov et al. [7] in Section 5] We believe that this algorithm is especially useful for domains with non-convex boundaries or different types of boundary conditions - leading to singularities in the solution at the boundary.

Another possible approach to the problem at hand is to include the leading order term of the error given in Corollary 3.9 for the purely reflecting case into the sample produced by the algorithm. Thus, the new leading order error term of the expansion is of higher order than the leading order term for the projected Euler method. We further develop this approach in Section 6, where we also encounter its limitations: the algorithm is straightforward only in dimension one, and even in this case it is not superior to the adaptive algorithm, again giving a rate of 0.8 .

\section{THE ADAPTIVE ALGORITHM FOR REFLECTED DIFFUSIONS}

The idea of adaptive algorithms for SDEs is the following: given a certain computational error that one is willing to tolerate, one wants to minimize the work while achieving a computational error smaller than the error tolerance. For "work" we substitute the number of time-steps used. In fact, since the number of time-steps will be random, we try to minimize the average number of time-steps. Notice that all our comments only refer to the algorithm discretizing the SDE in order to produce random samples for the final Monte Carlo simulation. For the general theory of adaptive weak algorithms for SDEs see Moon, Szepessy, Tempone and Zouraris [16].

First we present an adaptive algorithm for a purely reflected diffusion, i.e., based on the error expansion given in Corollary 3.9. In this situation, we can present a theory, partly based on numerical observations. Later we present a heuristic extension to the mixed problem, i.e., to the case with both Neumann and Dirichlet boundary conditions. As already mentioned before, the advantages of the adaptive algorithm are generally expected to show in situations with inherent singularities. We do not have a proof for a certain convergence rate, but notice that for problems with very low regularity most traditional weak convergence results fail, too.

Our starting point is the error expansion in Corollary 3.9, which we rewrite as

$$
E\left[\sum_{i=0}^{N-1}\left(\Delta \bar{Z}_{i}^{N}\right)^{2}\left|u_{n n}\left(t_{i+1}, \bar{X}_{i+1}^{N}, \Delta \bar{Z}_{i}^{N}\right)\right|\right]
$$

where we use the shorthand notation

$$
u_{n n}\left(t_{i+1}, \bar{X}_{i+1}^{N}, \Delta \bar{Z}_{i}^{N}\right)=\int_{0}^{1}(1-\theta) \frac{\partial^{2}}{\partial n\left(\bar{X}_{i+1}^{N}\right)^{2}} u\left(t_{i+1}, \bar{X}_{i+1}^{N}-\theta \Delta \bar{Z}_{i}^{N} n\left(\bar{X}_{i+1}^{N}\right)\right) d \theta .
$$

Define auxiliary functions $\Delta t^{N}:[0, T] \rightarrow \mathbb{R}$ by $\Delta t^{N}(t)=\Delta t_{i}=t_{i+1}-t_{i}$ and $\bar{Z}^{N}:[0, T] \rightarrow \mathbb{R}$ by $\bar{Z}^{N}(t)=\bar{Z}_{i+1}^{N}$ for $\left.\left.t \in\right] t_{i}, t_{i+1}\right]$. Let $\widetilde{N}$ denote the number of hits at the boundary for the given time grid. We approximate

$$
E[\widetilde{N}] \approx E\left[\int_{0}^{T} \frac{d \bar{Z}^{N}(t)}{\sqrt{\Delta t^{N}(t)}}\right]
$$

For justification of 4.2, we start with the same setting as in Lemma 3.11 
Lemma 4.1. Given a one-dimensional Brownian motion $\left(B_{t}\right)_{t \in[0, \infty[}$, a fixed time $T>0$ and a partition $0=t_{0}<\cdots<t_{N}=T$. Define, within the scope of this lemma,

$$
\Delta Z_{n}=\left(\left|B_{t_{n}}\right|+\operatorname{sign}\left(B_{t_{n}}\right) \Delta B_{n}\right),
$$

where, as usual, $\Delta B_{n}=B_{t_{n+1}}-B_{t_{n}}, \Delta t_{n}=t_{n+1}-t_{n}$ and $n \in\{0, \ldots, N-1\}$. Then

$$
\begin{aligned}
& E\left[\sum_{n=0}^{N-1} \mathbf{1}_{] 0, \infty[}\left(\Delta Z_{n}\right)\right] \leq 2 \sqrt{\frac{2}{\pi}} E\left[\sum_{n=0}^{N-1} \frac{\Delta Z_{n}}{\sqrt{\Delta t_{n}}}\right], \\
& E\left[\sum_{n=0}^{N-1} \mathbf{1}_{] 0, \infty[}\left(\Delta Z_{n}\right)\right] \geq \sqrt{\frac{\pi}{2}} E\left[\sum_{n=0}^{N-1} \frac{\Delta Z_{n}}{\sqrt{\Delta t_{n}}}\right] .
\end{aligned}
$$

Proof. By interchanging the summation with the expectation, we need to prove that

$$
P\left(\Delta Z_{n}>0\right) \leq(\geq) C E\left[\frac{\Delta Z_{n}}{\sqrt{\Delta t_{n}}}\right]
$$

for suitable constants $C$ as given in the statement of the lemma. For the computation of $P\left(\Delta Z_{n}>0\right)$, we proceed as in the proof of Lemma 3.11 with $\sqrt{n}$ replaced by $\sqrt{\frac{t_{n}}{\Delta t_{n}}}$, giving us

$$
P\left(\Delta Z_{n}>0\right)=\frac{1}{\pi} \arctan \left(\sqrt{\frac{\Delta t_{n}}{t_{n}}}\right) .
$$

Let $Y_{2}$ denote a standard Gaussian random variable. We have

$$
\begin{aligned}
E\left[\frac{\Delta Z_{n}}{\sqrt{\Delta t_{n}}} \mid B_{t_{n}}=x\right] & =E\left[\left(\frac{|x|}{\sqrt{\Delta t_{n}}}+\operatorname{sign}(x) Y_{2}\right)_{-}\right] \\
& =p\left(\frac{|x|}{\sqrt{\Delta t_{n}}}\right)-\frac{|x|}{\sqrt{\Delta t_{n}}}\left(1-\Phi\left(\frac{|x|}{\sqrt{\Delta t_{n}}}\right)\right),
\end{aligned}
$$

where $p$ denotes the density of the standard Gaussian law and $\Phi$ its cumulative distribution function. Consequently, we obtain

$$
\begin{aligned}
E\left[\frac{\Delta Z_{n}}{\sqrt{\Delta t_{n}}}\right] & =2 \int_{0}^{\infty}\left(p\left(\frac{\sqrt{t_{n}} x}{\sqrt{\Delta t_{n}}}\right)-\frac{\sqrt{t_{n}} x}{\sqrt{\Delta t_{n}}}\left(1-\Phi\left(\frac{\sqrt{t_{n}} x}{\sqrt{\Delta t_{n}}}\right)\right)\right) p(x) d x \\
& =\frac{1}{\sqrt{2 \pi}} \frac{1-\sqrt{\frac{t_{n}}{\Delta t_{n}}} \sqrt{\frac{t_{n}}{\Delta t_{n}}+1}+\frac{t_{n}}{\Delta t_{n}}}{\sqrt{\frac{t_{n}}{\Delta t_{n}}+1}} .
\end{aligned}
$$

Let $\delta:=\sqrt{\frac{t_{n}}{\Delta t_{n}}}$ and define

$$
f(\delta)=\frac{P\left(\Delta Z_{n}>0\right)}{E\left[\frac{\Delta Z_{n}}{\sqrt{\Delta t_{n}}}\right]}=\frac{\sqrt{2} \arctan (1 / \delta) \sqrt{1+\delta^{2}}}{\sqrt{\pi}\left(1-\delta \sqrt{1+\delta^{2}}+\delta^{2}\right)} .
$$

Noting the the numerator of $f$ is increasing in $\delta$, whereas the denominator is decreasing, we get that $f$ itself is an increasing function of $\delta$, with

$$
\lim _{\delta \searrow 0} f(\delta)=\sqrt{\frac{\pi}{2}} \approx 1.253, \quad \lim _{\delta \rightarrow \infty} f(\delta)=2 \sqrt{\frac{2}{\pi}} \approx 1.596 .
$$

Therefore, we can bound

$$
\begin{aligned}
& P\left(\Delta Z_{n}>0\right) \leq 2 \sqrt{\frac{2}{\pi}} E\left[\frac{\Delta Z_{n}}{\sqrt{\Delta t_{n}}}\right], \\
& P\left(\Delta Z_{n}>0\right) \geq \sqrt{\frac{\pi}{2}} E\left[\frac{\Delta Z_{n}}{\sqrt{\Delta t_{n}}}\right] .
\end{aligned}
$$

Summing up, we obtain the result. 
We want to minimize (4.2) subject to the constraint that the error term (4.1) is smaller than a given error tolerance TOL. In order to avoid non-adapted stochastic processes entering the picture at this stage, let us approximate the error term by its leading order term

$$
E\left[\sum_{i=0}^{N-1}\left(\Delta \bar{Z}_{i}^{N}\right)^{2}\left|u_{n n}\left(t_{i+1}, \bar{X}_{i+1}^{N}\right)\right|\right] \approx E\left[\sum_{i=0}^{N-1}\left|u_{n n}\left(t_{i+1}, \bar{X}_{i+1}^{N}\right)\right| \Delta \bar{Z}_{i}^{N} E\left(\Delta \bar{Z}_{i}^{N} \mid \bar{X}_{i}^{N}, \Delta t_{i}\right)\right] .
$$

Until we have identified an optimal strategy, let us assume that $\left(\Delta t_{i}\right)_{i=0}^{N-1}$ is an adapted timemesh in the sense that $\Delta t_{i}$ is measurable with respect to

$$
\mathcal{F}_{i}=\sigma\left(\left\{\bar{X}_{j} \mid j=0, \ldots, i\right\}\right)
$$

and such that $\Delta t_{0}+\cdots+\Delta t_{N-1}=T$. In particular, $N$ is constructed to be a random variable.

Lemma 4.2. Let $\psi(x, \Delta t)=E\left[\Delta \bar{Z}_{i} \mid \bar{X}_{i}=x, \Delta t_{i}=\Delta t\right]$. Assume that the domain $D$ is the half-space $D=\left\{x \mid\langle n, x\rangle \geq c_{D}\right\}$ for some normal vector $n$ with $\|n\|=1$, and that we consider the reflected diffusion (1.2). Denote by $\Phi$ and $p$ the distribution function and density of a standard normal distribution. Then

$$
\begin{gathered}
\psi(x, \Delta t)=A(x, \Delta t) \Phi\left(\frac{A(x, \Delta t)}{\sqrt{\Delta t} \sigma(x)}\right)+\sqrt{\Delta t} \sigma(x) p\left(\frac{A(x, \Delta t)}{\sqrt{\Delta t} \sigma(x)}\right), \\
\frac{\partial}{\partial \Delta t} \psi(x, \Delta t)=-\langle n, V(x)\rangle \Phi\left(\frac{A(x, \Delta t)}{\sqrt{\Delta t} \sigma(x)}\right)+\frac{\sigma(x)}{2 \sqrt{\Delta t}} p\left(\frac{A(x, \Delta t)}{\sqrt{\Delta t} \sigma(x)}\right),
\end{gathered}
$$

where

$$
A(x, \Delta t)=c_{D}-\langle n, x\rangle-\langle n, V(x)\rangle \Delta t, \quad \sigma(x)=\sqrt{\sum_{j=1}^{d}\left\langle n, V_{j}(x)\right\rangle^{2}} .
$$

Proof. Note that for $y \in D^{c}$, the projection onto $D$ is given by $\Pi(y)=y+\left(c_{D}-\langle n, y\rangle\right) n$, and the distance of $y$ to $\partial D$ is given by $c_{D}-\langle n, y\rangle$. Consequently,

$$
\begin{aligned}
\Delta \bar{Z}_{i} & =\left(c_{D}-\left\langle n, \hat{X}_{i+1}\right\rangle\right)_{+} \\
& =\left(c_{D}-\left\langle n, \bar{X}_{i}+V\left(\bar{X}_{i}\right) \Delta t_{i}+\sum_{j=0}^{d} V_{j}\left(\bar{X}_{i}\right) \Delta B^{j}\right\rangle\right)_{+} .
\end{aligned}
$$

Note that conditional on $\mathcal{F}_{i},\left\langle n, \sum_{j=0}^{d} V_{j}\left(\bar{X}_{i}\right) \Delta B^{j}\right\rangle$ has a normal distribution with variance $\sigma\left(\bar{X}_{i}\right)^{2} \Delta t_{i}$. Let $Y$ denote a $\mathcal{N}(0,1)$-distributed random variable independent of $\mathcal{F}_{i}$. Then we have

$$
\begin{aligned}
\psi(x, \Delta t) & =E\left[\left(c_{D}-\langle n, x\rangle-\langle n, V(x)\rangle \Delta t+\sqrt{\Delta t} \sigma(x) Y\right)_{+}\right] \\
& =\int_{-\frac{A(x, \Delta t)}{\sigma \sqrt{\Delta t}}}^{\infty}(A(x, \Delta t)+\sigma(x) \sqrt{\Delta t} y) p(y) d y \\
& =A(x, \Delta t) \Phi\left(\frac{A(x, \Delta t)}{\sqrt{\Delta t} \sigma(x)}\right)+\sqrt{\Delta t} \sigma(x) p\left(\frac{A(x, \Delta t)}{\sqrt{\Delta t} \sigma(x)}\right) .
\end{aligned}
$$

The formula for the derivative of $\psi$ is then given by differentiation of the above.

In the general case of a domain $D$ not given by a half-space, we locally approximate $D$ by a suitable (tangent) half-space with normal $n$ depending on $x$. Recall that we used $c_{D}-\langle n, x\rangle=-d(x)$ for $x \in D$, where $d(x)$ denotes the distance to the boundary $\partial D$. Therefore, we now again replace $c_{D}-\langle n(x), x\rangle$ with $d(x)$, i.e., we define $A$ as

$$
A(x, \Delta t)=-d(x)-\left\langle n\left(\Pi_{\partial D}(x)\right), V(x)\right\rangle \Delta t,
$$

where $\Pi_{\partial D}$ denotes the projection of $x$ to the boundary. 
Combining these results and rewriting everything in terms of integrals instead of sums, we get the Lagrangian of the minimization problem at hand:

$$
\mathcal{L}(\Delta t)=E\left[\int_{0}^{T} \frac{d \bar{Z}(s)}{\sqrt{\Delta t(s)}}+\lambda\left(\int_{0}^{T}\left|u_{n n}(s, \bar{X}(s))\right| \psi(\bar{X}(s), \Delta t(s)) d \bar{Z}(s)-\mathrm{TOL}\right)\right] .
$$

$\mathcal{L}$ is understood as a function defined on the set of positive, piecewise constant functions on $[0, T]$. Note that we have tacitly omitted the dependence on $N$ in the above equation, because $N$ is now a function of the mesh function $\Delta t$, which is no longer fixed. The derivative in direction of the piecewise constant function $\phi$ is then given by

$$
\mathcal{L}^{\prime}(\Delta t) \cdot \phi=E\left[\int_{0}^{T}\left(-\frac{1}{2 \Delta t(s)^{3 / 2}}+\lambda\left|u_{n n}(s, \bar{X}(s))\right| \frac{\partial}{\partial \Delta t(s)} \psi(\bar{X}(s), \Delta t(s))\right) \phi(s) d \bar{Z}(s)\right] .
$$

The condition that $\mathcal{L}^{\prime}(\Delta t) \cdot \phi=0$ for all functions $\phi$ implies

$$
\begin{aligned}
\text { const }=\frac{1}{\lambda}=[-2\langle n(\bar{X}(t)), V(\bar{X}(t))\rangle & \Phi\left(\frac{A(\bar{X}(t), \Delta t(t))}{\sqrt{\Delta t(t)} \sigma(\bar{X}(t))}\right) \Delta t(t)^{3 / 2}+ \\
& \left.+\sigma(\bar{X}(t)) p\left(\frac{A(\bar{X}(t), \Delta t(t))}{\sqrt{\Delta t(t)} \sigma(\bar{X}(t))}\right) \Delta t(t)\right]\left|u_{n n}(t, \bar{X}(t))\right|
\end{aligned}
$$

for all $t \in[0, T]$. Notice that 4.8 can already be understood as a refinement rule, because it shows the dependence of the grid on the position. There is, however, no direct dependence on the tolerance level TOL included, yet. To this end, we have the re-insert the expression (4.8) into the error representation (4.5), giving us the constant in (4.8). More precisely, note that

$$
\begin{aligned}
\frac{1}{\lambda}=\sqrt{\Delta t(t)} \psi & (\bar{X}(t), \Delta t(t))\left|u_{n n}(t, \bar{X}(t))\right|- \\
- & \underbrace{(-d(\bar{X}(t))+\langle n(\bar{X}(t)), V(\bar{X}(t))\rangle \Delta t(t))}_{=: B(\bar{X}(t), \Delta t(t))} \Phi\left(\frac{A(\bar{X}(t), \Delta t(t))}{\sqrt{\Delta t(t)} \sigma(\bar{X}(t))}\right)\left|u_{n n}(t, \bar{X}(t))\right| .
\end{aligned}
$$

Therefore, plugging (4.8) into the constraint yields

$$
\begin{aligned}
& \text { 9) } \begin{array}{r}
\text { TOL }=E\left[\int_{0}^{T} \frac{\left|u_{n n}(s, \bar{X}(s))\right| B(\bar{X}(s), \Delta t(s)) \Phi\left(\frac{A(\bar{X}(t), \Delta t(t))}{\sqrt{\Delta t(t)} \sigma(\bar{X}(t))}\right)}{\sqrt{\Delta t(s)}} d \bar{Z}(s)\right]+ \\
+\left[\Delta t(t) \sigma(\bar{X}(t)) p\left(\frac{A(\bar{X}(t), \Delta t(t))}{\sqrt{\Delta t(t)}}\right)-2 \Delta t(t)^{3 / 2}\langle n(\bar{X}(t)), V(\bar{X}(t))\rangle \Phi\left(\frac{A(\bar{X}(t), \Delta t(t))}{\sqrt{\Delta t(t)}}\right)\right] \times \\
\times\left|u_{n n}(t, \bar{X}(t))\right| E\left[\int_{0}^{T} \frac{d \bar{Z}(s)}{\sqrt{\Delta t(s)}}\right]
\end{array}
\end{aligned}
$$

This term is too complicated for the implementation. Two simplifications are immediately possible. Note that

$$
B(x, \Delta t)=\underbrace{-d(x)}_{\leq 0}+\langle n(x), V(x)\rangle \Delta t .
$$

Therefore, the positive part of $B$ is small. If $V$ is small close to the boundary $\partial D$, we may, therefore, ignore the first term in 4.9. . Otherwise, we can run the adaptive algorithm once in order to get an estimate for the expected value, and then run the algorithm again using that estimate. If necessary, this procedure can be iterated. Secondly, we may again apply (4.2), this time in the other direction, which gives a term $E(\widetilde{N})$ in the denominator. In practice, we do not know this expected value, especially since it highly depends on the grid, which is no longer fixed. Therefore, we can use the same procedure as for the first expectation. However, empirically it turns out that the expected number of hits as a 
function of TOL can be reliably estimated, and then used for the computations. Moreover, the term of order $\Delta t(t)^{3 / 2}$ is negligible for small $\Delta t$. These simplifications lead to

$$
\begin{aligned}
\mathrm{TOL} & =\Delta t(t) \sigma(\bar{X}(t)) p\left(\frac{A(\bar{X}(t), \Delta t(t))}{\sqrt{\Delta t(t)}}\right)\left|u_{n n}(t, \bar{X}(t))\right| E[\widetilde{N}] \\
& \approx \Delta t(t) \sigma(\bar{X}(t)) p\left(\frac{d(\bar{X}(t))}{\sqrt{\Delta t(t)}}\right)\left|u_{n n}(t, \bar{X}(t))\right| E[\widetilde{N}] .
\end{aligned}
$$

Next we solve the equation 4.10 for the optimal increment $\Delta t$ using a special function known as Lambert's W-function, see [5]. This function is defined by

$$
W(z) \exp (W(z))=z .
$$

The following elementary facts about Lambert's W-function are presented for later use.

Lemma 4.3. The function $f(x)=\frac{W(x)}{x}, x \geq 0$, is strictly decreasing in $x$, positive and $f(0)=1$. Moreover, for $x \geq e, W(x) \leq \log (x)$.

Proof. First note that $W(x)$ itself is strictly increasing on $\mathbb{R}_{\geq 0}$. The last two assertions on $f$ follow immediately from

$$
f(x)=e^{-W(x)}
$$

The monotonicity follows just as easily from

$$
f^{\prime}(x)=-e^{-W(x)} W^{\prime}(x) .
$$

Let us write $W(x)=\log (x)+g(x)$. Then at $x \geq e$ we have $x=W(x) \cdot x \cdot e^{g(x)}$, thus $1=W(x) e^{g(x)}$. Since $W(x) \geq 1$ for $x \geq e$, we have $g(x) \leq 0$.

Lemma 4.4. The reduced equation (4.10) for the optimal time steps corresponds to the explicit formula

$$
\Delta t_{i}=\frac{d\left(\bar{X}_{i}\right)^{2}}{2 \sigma\left(\bar{X}_{i}\right)^{2} W\left(\frac{d\left(\bar{X}_{i}\right)^{2}\left|u_{n n}\left(t_{i} \bar{X}_{i}\right)\right| E[\widetilde{N}]}{\sqrt{2 \pi} 2 \sigma\left(\bar{X}_{i}\right) \mathrm{TOL}}\right)},
$$

where $d(x)$ denotes the distance of $x \in D$ to the boundary $\partial D$.

Proof. Equation 4.10) is equivalent to

$$
\exp \left(\frac{d\left(\bar{X}_{i}\right)}{2 \sigma\left(\bar{X}_{i}\right)^{2} \Delta t}\right) \frac{d\left(\bar{X}_{i}\right)^{2}}{2 \sigma\left(\bar{X}_{i}\right)^{2} \Delta t_{i}}=\frac{d\left(\bar{X}_{i}\right)^{2}}{2 \sigma\left(\bar{X}_{i}\right) \text { TOL } \sqrt{2 \pi}}\left|u_{n n}\left(t_{i}, \bar{X}_{i}\right)\right| E[\widetilde{N}],
$$

for $d\left(\bar{X}_{i}\right)>0$. The formula 4.11 now follows by definition of Lambert's W-function. For $d\left(\bar{X}_{i}\right)=0$ the formula is still valid by continuity of 4.11.

There is still the unknown term $\left|u_{n n}(t, \bar{X}(t))\right|$ left in equation 4.10. We propose two possible approaches to it, depending on the problem at hand.

- If the problem is simple enough or we have some a-priori information, we can replace $\left|u_{n n}(t, \bar{X}(t))\right|$ by the constant 1 or by some approximation based on our knowledge of the problem, respectively. For instance, if $v$ is well behaved but for one singularity at a known point $x_{0} \in \mathbb{R}^{n}$, we could replace $\left|u_{n n}(t, \bar{X}(t))\right|$ with 1 far away from $x_{0}$ and with $1 /\left\|x-x_{0}\right\|^{\alpha}$ close to $x_{0}$, with $\alpha$ depending on the type of singularity. Notice, however, that in this situation the true computational error can only be proportional to the error estimate given by the local error indicator (4.10). Therefore, it is not possible to guarantee - within the limitations of a Monte-Carlo setup and our further assumptions - the computational error to be bounded by TOL, but one has to observe the dynamics of the results for smaller and smaller TOL. 
- In general, $\left|u_{n n}(t, \bar{X}(t))\right|$ can be approximated by the discrete dual functions, see [20] for more details. The use of the dual functions introduces some subtleties into the algorithm, because they are computed by backward recursion. Thus, they are not adapted to the filtration. In this case, the error representation based on the local error indicator is precise, again under the limitations of the setup, in particular keeping in mind that the discrete dual functions are only approximations of the true second normal derivative.

Let us summarize the adaptive algorithm. In the following, $t$ will denote a partition of $[0, T]$, i.e., $\mathrm{t}=\left(t_{i}\right)_{i=0}^{N(\mathrm{t})}$ with $0=t_{0}<\cdots<t_{N(\mathrm{t})}=T$. Given a partition $\mathrm{t}$, we will denote $\Delta t_{i}^{\mathrm{t}}=t_{i+1}-t_{i}, 0 \leq i \leq N(\mathrm{t})-1$. Moreover, $\bar{B}^{\mathrm{t}}, \bar{X}^{\mathrm{t}}, \bar{Z}^{\mathrm{t}}$ will denote discrete processes defined on the grid t, i.e., $\bar{B}^{\mathrm{t}}=\left(\bar{B}_{t_{i}}\right)_{i=0}^{N(t)}$ and we will also use $\Delta \bar{B}_{i}^{\mathrm{t}}=\bar{B}_{t_{i+1}}-\bar{B}_{t_{i}}, \Delta \bar{Z}_{i}^{\mathrm{t}}=\bar{Z}_{i+1}^{\mathrm{t}}-\bar{Z}_{i}^{\mathrm{t}}$, $0 \leq i \leq N(\mathrm{t})-1$.

Remark 4.5. Of course, it is also possible to sample the Brownian motion in a weak way. In this situation, one has to use the weak analogue of the above Brownian bridge formula.

Algorithm 4.6 (Adaptive Euler Algorithm for Reflecting Diffusions - Refinement Version). Start with an initial mesh $\mathrm{t}$ and the corresponding Brownian motion $\bar{B}^{t}$.

(1) $\operatorname{Set} \bar{X}_{0}^{\dagger}=x, \bar{Z}_{0}^{\dagger}=0$, and $i=0$.

(2) Set

$$
\hat{X}_{i+1}^{\dagger}=\bar{X}_{i}^{\dagger}+V\left(\bar{X}_{i}^{\dagger}\right) \Delta t_{i}+\sum_{j=1}^{d} V_{j}\left(\bar{X}_{i}^{\dagger}\right) \Delta \bar{B}_{i}^{j}
$$

(3) Set

$$
\begin{aligned}
& \bar{X}_{i+1}^{t}= \begin{cases}\hat{X}_{i+1}^{\dagger}, & \hat{X}_{i+1}^{\dagger} \in \bar{D}, \\
\Pi\left(\hat{X}_{i+1}^{\dagger}\right), & \hat{X}_{i+1}^{\dagger} \notin \bar{D},\end{cases} \\
& \bar{Z}_{i+1}^{\mathrm{t}}= \begin{cases}\bar{Z}_{i}^{\dagger}, & \hat{X}_{i+1}^{\dagger} \in \bar{D}, \\
\bar{Z}_{i}^{\dagger}+\left\|\Pi\left(\hat{X}_{i+1}^{\dagger}\right)-\hat{X}_{i+1}^{\dagger}\right\|, & \hat{X}_{i+1}^{\dagger} \notin \bar{D} .\end{cases}
\end{aligned}
$$

(4) Increase $i$ by one. If $i<N(\mathrm{t})$, go back to (2).

(5) Refine the grid $\mathrm{t}$ using Algorithm 4.7 If the grid has changed during refinement, then set $i=0$ and go back to (2), using the refined grid.

(6) Calculate

$$
\bar{F}^{\mathrm{t}}=f\left(\bar{X}_{N(\mathrm{t})}^{\mathrm{t}}\right)-\sum_{i=0}^{N(\mathrm{t})-1} h\left(\bar{X}_{i+1}^{\mathrm{t}}\right) \Delta \bar{Z}_{i}^{\mathrm{t}}
$$

Algorithm 4.7 (Refinement Algorithm). Given a time grid $\mathrm{t}$, the Brownian motion $B^{\mathrm{t}}$ sampled on $\mathrm{t}$ and an error tolerance TOL. Do for $i=0, \ldots, N(\mathrm{t})-1$ :

(1) Compute a local error indicator by

$$
l_{i}=\Delta t_{i} \sigma\left(\bar{X}_{i}\right) p\left(\frac{A\left(\bar{X}_{i}, \Delta t_{i}\right)}{\sqrt{\Delta t_{i}}}\right)\left|u_{n n}\left(t_{i}, \bar{X}_{i}\right)\right| E[\widetilde{N}],
$$

where $\left|u_{n n}\left(t_{i}, \bar{X}_{i}\right)\right|$ is chosen by one of the two approaches mentioned earlier.

(2) If $l_{i}>\mathrm{TOL}$, insert $t=\frac{t_{i}+t_{i+1}}{2}$ into the grid $\mathrm{t}$ and generate the corresponding sample of the Brownian motion $B_{t}$ by a Brownian bridge between the already sample values $B_{t_{i}}$ and $B_{t_{i+1}}$.

As an alternative, we can directly use the explicit formula 4.11, which greatly increases the efficiency of the algorithm. The corresponding Euler scheme has only a marginally higher complexity as a uniform Euler scheme with a grid size equal to the mean grid size of the adaptive scheme. 
Algorithm 4.8 (Adaptive Euler Algorithm for Reflecting Diffusions - Explicit Version). Start with $\bar{X}_{0}=x_{0}$ and set $t=0, i=0, N=0$ and $B_{0}=0$. While $t<T$ do:

(1) Define $\Delta t_{i}$ by equation (4.11) (but not larger then $T-t$ ) and generate $\Delta B_{i}$ accordingly, i.e., as a vector of $d$ independent normals with variance $\Delta t_{i}$.

(2) Set

$$
\hat{X}_{i+1}=\bar{X}_{i}+V\left(\bar{X}_{i}\right) \Delta t_{i}+\sum_{j=1}^{d} V_{j}\left(\bar{X}_{i}\right) \Delta \bar{B}_{i}^{j} .
$$

(3) $\mathrm{Set}$

$$
\begin{aligned}
\bar{X}_{i+1} & = \begin{cases}\hat{X}_{i+1}, & \hat{X}_{i+1} \in \bar{D}, \\
\Pi\left(\hat{X}_{i+1}\right), & \hat{X}_{i+1} \notin \bar{D},\end{cases} \\
\Delta \bar{Z}_{i} & = \begin{cases}0, & \hat{X}_{i+1} \in \bar{D}, \\
\| \Pi\left(\hat{X}_{i+1}-\hat{X}_{i+1} \|,\right. & \hat{X}_{i+1} \notin \bar{D} .\end{cases}
\end{aligned}
$$

(4) Increase $i$ and $N$ by one and update $t=t+\Delta t$.

Finally, calculate

$$
\bar{F}=f\left(\bar{X}_{N}\right)-\sum_{i=0}^{N-1} h\left(\bar{X}_{i+1}\right) \Delta \bar{Z}_{i} .
$$

Remark 4.9. Algorithm 4.7 uses the reduced form of 4.9], where the first expected value is removed. Alternatively, one could use the full form 4.9 and estimate the expected value in that formula in a first run, using the so obtained value in a second run for the computation of the error density.

Remark 4.10. In both algorithms, knowledge of $E[\widetilde{N}]$ is presupposed. One can either use an asymptotic formula $E[\widetilde{N}] \approx \mathrm{TOL}^{-\beta}$ for some numerically computed constant $\beta$ as discussed below, or one can use the same approach as advocated in Remark 4.9

Remark 4.11. Both Algorithm 4.6 and Algorithm 4.8 ignore the error contribution in the interior of the domain, i.e., they ignore the part

$$
E\left[\int_{0}^{T}(\bar{L}-L) u\left(t, \bar{X}_{\lfloor t\rfloor}\right) d t\right] .
$$

One can construct an adaptive algorithm also using this "interior" error contribution based on the theory developed in [20] and [16] - in the same fashion as we combine the algorithms for reflecting and for stopped diffusions in Section 5 On the other hand, since the adaptive algorithms for reflecting diffusions have an order of weak convergence less than one, cf. Remark 4.17 we may ignore the interior error contribution as a higher order error term, provided that the initial mesh is fine enough, e.g., uniform with $\Delta t \approx$ TOL.

Using the adaptive Algorithm 4.8 , we know that the discretization error is of order TOL, provided some assumptions. However, in order to obtain a convergence rate we need to compute the work in the sense of the size of the finest grid in the adaptive algorithm in terms of TOL. Since the number of hits at the boundary $E[\widetilde{N}]$ undoubtedly depends strongly on TOL, too, we need to get a grip on its dependence on TOL before being able to compute the dependence of the total work. The following assumption is based on numerical evidence.

Assumption 4.12. The expected number of hits at the boundary depends on the tolerance as

for some $\beta>0$.

$$
E(\widetilde{N}) \lesssim \mathrm{TOL}^{-\beta}
$$

Remark 4.13. Numerical experiments suggest $\beta \approx 5 / 4$ in many cases, see the numerical part of this paper. However, the optimal rate $\beta$ might not be problem independent 
By Lemma 4.3 , the optimal time-steps $\Delta t_{i}$ given by Lemma 4.4 are asymptotically (in TOL) bounded from below by

$$
\Delta t_{\mathrm{MIN}}=\frac{\sqrt{2 \pi} \mathrm{TOL}^{1+\beta}}{\left\|\left.u_{n n}\right|_{\partial D}\right\|_{\infty}\left\|\left.\sigma\right|_{\partial D}\right\|_{\infty}},
$$

given Assumption 4.12 We also need a boundedness assumption on the density of the law of the Euler approximations $\bar{X}$ away from the boundary.

Assumption 4.14. The densities $p^{\mathrm{TOL}}(t, x)$ of $\bar{X}_{\lfloor t\rfloor}$ on $\bar{X}_{\lfloor t\rfloor}>0$ are uniformly bounded for TOL $\rightarrow 0$.

For the next lemma, we again confine ourselves to the situation of reflection at a halfspace, without loss of generality given by the normal vector $n$.

Proposition 4.15. For the approximation of $(1.1)$ on a half-space domain using Algorithm 4.8 take Assumption 4.12 and Assumption 4.14 for granted and assume that the second normal derivative of of the solution and $\sigma(x)^{2}=\sum_{j=1}^{d}\left\langle n, V_{j}(x)\right\rangle^{2}$ are uniformly bounded away from zero as well as from above. Moreover, assume that $\langle n, V(x)\rangle$ is uniformly bounded. Then the work in the sense of the expected value of the final grid size is asymptotically bounded by

$$
E(N) \lesssim \mathrm{TOL}^{-\max (\beta,(1+\beta) / 2)} .
$$

Proof. Fix TOL $>0$ and $\delta>0$ possibly depending on TOL. Then obviously

$$
E(N)=E\left[\sum_{i=0}^{N-1} \mathbf{1}_{\left\{d\left(\bar{X}_{i}\right)<\delta\right\}}\right]+E\left[\sum_{i=0}^{N-1} \mathbf{1}_{\left\{d\left(\bar{X}_{i}\right) \geq \delta\right\}}\right] .
$$

Let us first consider the second expectation above, i.e., the sum over "large" values of $d\left(\bar{X}_{i}\right)$. In order to being able to interchange integration and summation, we move to a continuous formulation of the formula, i.e.,

$$
\begin{aligned}
E\left[\sum_{i=0}^{N-1} \mathbf{1}_{\left.\left\{d\left(\bar{X}_{i}\right) \geq \delta\right\}\right]}\right] & =E\left[\sum_{i=0}^{N-1} \mathbf{1}_{\left\{d\left(\bar{X}_{i}\right) \geq \delta\right\}} \frac{\Delta t_{i}}{\Delta t_{i}}\right] \\
& =E\left[\int_{0}^{T} \mathbf{1}_{\left\{d\left(\bar{X}_{\lfloor s]}\right) \geq \delta\right\}} \frac{1}{\Delta t(s)} d s\right],
\end{aligned}
$$

where $\lfloor s\rfloor=\sup \left\{0 \leq i \leq N \mid t_{i} \leq s\right\}$ and $\Delta t(s)=\Delta t_{\lfloor s\rfloor}$. Note that both $\lfloor s\rfloor$ and $\Delta t(s)$ are piecewise constant stochastic processes, and $\Delta t(s)$ is asymptotically bounded from below by $\Delta t_{\text {MIN }}$ given in $(4.12$ ). We may interchange the expectation and the integration with respect to time by Fubini's theorem. Next we provide a bound for the expected value of the integrand. We replace $\Delta t_{\lfloor s\rfloor}$ using (4.11) and get

$$
\begin{aligned}
E\left[\mathbf{1}_{\left\{d\left(\bar{X}_{\lfloor s\rfloor}\right) \geq \delta\right\}} \frac{1}{\Delta t(s)}\right] & \leq E\left[\mathbf{1}_{\left\{d\left(\bar{X}_{\lfloor s s}\right\}\right.} \frac{2 \sigma\left(\bar{X}_{\lfloor s\rfloor}\right) W\left(\frac{d\left(\bar{X}_{\lfloor s\rfloor}\right)^{2}\left|u_{n n}\left(t_{\lfloor s\rfloor}, \bar{X}_{\lfloor s s}\right)\right| \mathrm{TOL}^{-(1+\beta)}}{2 \sqrt{2 \pi} \sigma\left(\bar{X}_{\lfloor s s}\right)}\right)}{d\left(\bar{X}_{\lfloor s\rfloor}\right)^{2}}\right] \\
& \leq E\left[\mathbf{1}_{\left\{d\left(\bar{X}_{\lfloor s\rfloor} \geq \delta\right\}\right.} \frac{2 \log \left(\frac{d\left(\bar{X}_{\lfloor s\rfloor}\right)^{2} \mid u_{n n}\left(t_{\lfloor s s}, \bar{X}_{\lfloor s s} \mid \mathrm{TOL}^{-(1+\beta)}\right)}{2 \sqrt{2 \pi} \sigma\left(\bar{X}_{\lfloor s s}\right)}\right)}{\bar{X}_{\lfloor s\rfloor}^{2}}\right],
\end{aligned}
$$

where the inequality is valid by Lemma 4.3 provided that

$$
\delta=\sqrt{2 \sqrt{2 \pi} e\|\sigma\|_{\infty}\left\|u_{n n}^{-1}\right\|_{\infty}} \mathrm{TOL}^{(1+\beta) / 2}=: C_{1} \mathrm{TOL}^{(1+\beta) / 2} .
$$


Thus, we may further simplify, ignoring constants and using the assumption that $\bar{X}_{\lfloor s\rfloor}$, and thus $d\left(\bar{X}_{\lfloor s\rfloor}\right)$ has a bounded density (bounded by $c>0$ ),

$$
\begin{aligned}
E\left[\mathbf{1}_{\left\{d\left(\bar{X}_{\lfloor s]}\right) \geq \delta\right\}} \frac{1}{\Delta t(s)}\right] & \leq c \int_{\delta}^{\infty} \frac{2 \log \left(\frac{y^{2}\left\|u_{n n}\right\|_{\infty}\left\|\sigma^{-1}\right\|_{\infty} \mathrm{TOL}^{-(1+\beta)}}{2 \sqrt{2 \pi}}\right)}{y^{2}} d y \\
& =\frac{2 c}{C_{1}}\left[2+\log \left(\frac{C_{1}^{2}\left\|u_{n n}\right\|_{\infty}\left\|\sigma^{-1}\right\|_{\infty}}{2 \sqrt{2 \pi}}\right)\right] \mathrm{TOL}^{-(1+\beta) / 2} \\
& \approx \mathrm{TOL}^{-(1+\beta) / 2}
\end{aligned}
$$

where we have used that the primitive function of $\frac{\log \left(a y^{2}\right)}{y^{2}}$ is given by $-\frac{2+\log \left(a y^{2}\right)}{y}$. Note that the above formula is independent from the index $i$ and from time, therefore we obtain

$$
E\left[\sum_{i=0}^{N-1} \mathbf{1}_{\left\{d\left(\bar{X}_{i}\right) \geq \delta\right\}}\right] \lesssim T \mathrm{TOL}^{-\frac{1+\beta}{2}}
$$

For the first sum, the number of steps with $\bar{X}_{i}<\delta$, we proceed by making yet more subdivisions of the form

$$
\begin{aligned}
& E\left[\sum_{i=0}^{N-1} \mathbf{1}_{\left\{0 \leq d\left(\bar{X}_{i}\right)<\delta\right\}}\right]=E\left[\sum_{i=0}^{N-1} \mathbf{1}_{\left\{d\left(\bar{X}_{i}\right)=0\right\}}\right]+E\left[\sum_{i=0}^{N-1} \mathbf{1}_{\left\{0<d\left(\bar{X}_{i}\right)<\delta\right\}}\right] \\
& =E[\widetilde{N}]+E\left[\sum_{i=0}^{N-1} \mathbf{1}_{\left\{0<d\left(\bar{X}_{i}\right)<\delta\right\} \cap\left\{d\left(\bar{X}_{i+1}\right)=0\right\}}\right]+E\left[\sum_{i=0}^{N-1} \mathbf{1}_{\left.\left\{0<d\left(\bar{X}_{i}\right)<\delta\right\} \cap\left\{d\left(\bar{X}_{i+1}\right) \geq \delta\right\}\right]}\right]+ \\
& +E\left[\sum_{i=0}^{N-1} \mathbf{1}_{\left\{0<d\left(\bar{X}_{i}\right)<\delta\right\} \cap\left\{0<d\left(\bar{X}_{i+1}\right)<\delta\right\}}\right]
\end{aligned}
$$

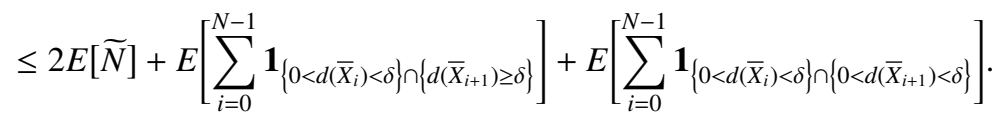

Noting that the random time $t_{i}$ is a stopping time for $i \in \mathbb{N}$ and the fact that the set $\{i<N\}$ is $\mathcal{F}_{t_{i}}$-measurable, we can re-phrase the second expectation as

$$
\begin{aligned}
E\left[\sum_{i=0}^{N-1} \mathbf{1}_{\left\{0<d\left(\bar{X}_{i}\right)<\delta\right\} \cap\left\{d\left(\bar{X}_{i+1}\right) \geq \delta\right\}}\right] & =E\left[\sum_{i=0}^{\infty} \mathbf{1}_{\left\{0<d\left(\bar{X}_{i}\right)<\delta\right\} \cap\left\{d\left(\bar{X}_{i+1}\right) \geq \delta\right\}} \mathbf{1}_{\{i<N\}}\right] \\
& =E\left[\sum_{i=0}^{N-1} P\left[d\left(\bar{X}_{i+1}\right) \geq \delta \mid \mathcal{F}_{t_{i}}\right] \mathbf{1}_{\left\{0<d\left(\bar{X}_{i}\right)<\delta\right\}}\right] .
\end{aligned}
$$

Note that for the half-space under consideration, $d(x)=\langle n, x\rangle, x \in \bar{D}$. For a standard Gaussian random variable $Y$, the above conditional probability can be expressed using

$$
\begin{aligned}
P\left[d\left(\bar{X}_{i+1}\right) \geq \delta \mid \bar{X}_{i}=x\right] & =P[\langle n, x+V(x) \Delta t(x)\rangle+\sigma(x) \sqrt{\Delta t(x)} Y \geq \delta] \\
& =\Phi\left(-\frac{\delta-\langle n, x+V(x) \Delta t(x)\rangle}{\sigma(x) \sqrt{\Delta t(x)}}\right)
\end{aligned}
$$

where $\Phi$ denotes the cumulative distribution function of the standard normal distribution and $\Delta t(x)$ denotes $\Delta t_{i}$ as a function of $\bar{X}_{i}=x$. As before, $\sigma$ is defined by

$$
\sigma(x)^{2}=\sum_{j=1}^{d}\left\langle n, V_{j}(x)\right\rangle^{2} .
$$


For $0<d(x)<\delta$ and $\delta=C_{1} \mathrm{TOL}^{(1+\beta) / 2}$ by 4.13 , we introduce $\tilde{x}:=x \mathrm{TOL}^{-(1+\beta) / 2}$ and $\widetilde{\Delta t}:=\Delta t(x) \mathrm{TOL}^{-(1+\beta)}$ and note that

$$
0<\widetilde{x}<C_{1}, \quad 0<\frac{\sqrt{2 \pi}}{\left\|u_{n n}\right\|_{\infty}\|\sigma\|_{\infty}} \leq \widetilde{\Delta t} \leq \frac{C_{1}^{2}\left\|\sigma^{-1}\right\|_{\infty}^{2}}{2 W\left(\frac{C_{1}^{2}}{2 \sqrt{2 \pi}\|\sigma\|_{\infty}\left\|u_{n n}^{-1}\right\|_{\infty}}\right)},
$$

at least for TOL small enough. This gives

$$
P\left[d\left(\bar{X}_{i+1}\right) \geq \delta \mid \bar{X}_{i}=x\right]=\Phi(-\frac{C_{1}-\langle n, \widetilde{x}\rangle}{\sigma(x) \sqrt{\widetilde{\Delta}}}+\underbrace{\left.\frac{\langle n, V(x)\rangle \sqrt{\widetilde{\Delta t}} \mathrm{TOL}^{(1+\beta) / 2}}{\sigma(x)}\right)}_{=O\left(\mathrm{TOL}^{(1+\beta) / 2)}\right)}, \quad 0<d(x)<\delta,
$$

by boundedness of $\langle n, V(x)\rangle$. Similarly, we obtain

$$
P\left[d\left(\bar{X}_{i+1}\right)=0 \mid \bar{X}_{i}=x\right]=\Phi(-\frac{\langle n, \widetilde{x}\rangle}{\sigma(x) \sqrt{\widetilde{\Delta}}}-\underbrace{\frac{\langle n, V(x)\rangle \sqrt{\widetilde{\Delta}} \mathrm{TOL}^{(1+\beta) / 2}}{\sigma(x)}}_{=O\left(\mathrm{TOL}^{(1+\beta) / 2)}\right)}), \quad 0<d(x)<\delta .
$$

Therefore,

$$
\begin{aligned}
P\left[d\left(\bar{X}_{i+1}\right) \geq \delta \mid \bar{X}_{i}=x\right] & \lesssim \sup \left\{\Phi\left(-\frac{C_{1}-\langle n, \widetilde{x}\rangle}{\sigma(x) \sqrt{\widetilde{\Delta t}}}\right) \mid \Phi\left(-\frac{\langle n, \widetilde{x}\rangle}{\sigma(x) \sqrt{\widetilde{\Delta}}}\right)\right\} P\left[d\left(\bar{X}_{i+1}\right)=0 \mid \bar{X}_{i}=x\right] \\
& \leq C_{2} P\left[d\left(\bar{X}_{i+1}\right)=0 \mid \bar{X}_{i}=x\right]
\end{aligned}
$$

uniformly in $0<d(x)<\delta$, where

$$
C_{2}:=\frac{1}{2 \Phi\left(-\frac{C_{1}\left\|\sigma^{-1}\right\|_{\infty} \sqrt{\left\|u_{n n}\right\|_{\infty}\|\sigma\|_{\infty}}}{(2 \pi)^{1 / 4}}\right)}<\infty .
$$

Consequently, the second sum in 4.15 can be bounded from above by

$$
E\left[\sum_{i=0}^{N-1} \mathbf{1}_{\left\{0<d\left(\bar{X}_{i}\right)<\delta\right\} \cap\left\{d\left(\bar{X}_{i+1}\right) \geq \delta\right\}}\right] \lesssim C_{2} E\left[\sum_{i=0}^{N-1} P\left[d\left(\bar{X}_{i+1}\right)=0 \mid \mathcal{F}_{t_{i}}\right] \mathbf{1}_{\left\{0<d\left(\bar{X}_{i}\right)<\delta\right\}}\right] \leq C_{2} E[\widetilde{N}] .
$$

For the third sum of 4.15 we proceed in a similar way as for the second one. Using the notations introduced before, we have

$$
P\left[0<d\left(\bar{X}_{i+1}\right)<\delta \mid \bar{X}_{i}=x\right] \geqq \Phi\left(\frac{C_{1}-\langle n, \widetilde{x}\rangle}{\sigma(x) \sqrt{\widetilde{\Delta t}}}\right)-\Phi\left(-\frac{\langle n, \widetilde{x}\rangle}{\sigma(x) \sqrt{\widetilde{\Delta t}}}\right) \leq C_{3}, \quad 0<x<\delta,
$$

where

$$
C_{3}:=2 \Phi\left(\frac{C_{1}\left\|\sigma^{-1}\right\|_{\infty} \sqrt{\left\|u_{n n}\right\|_{\infty}} \sqrt{\|\sigma\|_{\infty}}}{(2 \pi)^{1 / 4}}\right)-1<1 .
$$

This implies that the last term in 4.15) can finally be bounded from above by

$$
E\left[\sum_{i=0}^{N-1} \mathbf{1}_{\left\{0<d\left(\bar{X}_{i}\right)<\delta\right\} \cap\left\{0<d\left(\bar{X}_{i+1}\right)<\delta\right\}}\right] \lesssim C_{3} E\left[\sum_{i=0}^{N-1} \mathbf{1}_{\left\{0<d\left(\bar{X}_{i}\right)<\delta\right\}}\right] \leq C_{3} E[N] .
$$

Inserting the bounds 4.17) and 4.19) back into 4.15, we obtain

$$
E\left[\sum_{i=0}^{N-1} \mathbf{1}_{\left\{0 \leq \bar{X}_{i}<\delta\right\}}\right] \lesssim\left(2+C_{2}\right) E[\widetilde{N}]+C_{3} E[N] .
$$

Together with 4.14, the expected total work can, thus, be bounded by

$$
E[N] \lesssim \text { const } \mathrm{TOL}^{-(1+\beta) / 2}+\left(2+C_{2}\right) E[\widetilde{N}]+C_{3} E[N],
$$


which, by $C_{3}<1$, implies that

$$
E[N] \lesssim \frac{1}{1-C_{3}}\left(\text { const } \mathrm{TOL}^{-(1+\beta) / 2}+\left(2+C_{2}\right) E[\widetilde{N}]\right) .
$$

Remark 4.16. The proof of Proposition 4.15 can be immediately extended to general domains $D$ (satisfying the usual conditions for reflecting diffusions) provided that one can show that

$$
\begin{aligned}
& \sup \left\{\frac{P\left[d\left(\bar{X}_{i+1}\right) \geq \delta \mid \bar{X}_{i}=x\right]}{P\left[d\left(\bar{X}_{i+1}\right)=0 \mid \bar{X}_{i}=x\right]} \mid 0<d(x)<\delta=C_{1} \mathrm{TOL}^{(1+\beta) / 2}, 0<\mathrm{TOL}<\mathrm{TOL}_{0}\right\}<\infty, \\
& \sup \left\{P\left[0<d\left(\bar{X}_{i+1}\right)<\delta \mid \bar{X}_{i}=x\right] \mid 0<d(x)<\delta=C_{1} \mathrm{TOL}^{(1+\beta) / 2}, 0<\mathrm{TOL}<\mathrm{TOL}_{0}\right\}<1,
\end{aligned}
$$

for some $\mathrm{TOL}_{0}>0$.

Remark 4.17. Under the assumptions of Proposition 4.15 provided that indeed Error $\approx$ TOL, the order of convergence of the method is given by

$$
\text { Error } \approx E(N)^{-1 / \max (\beta,(1+\beta) / 2)} \text {. }
$$

If, as indicated by computer-experiments. $\beta \approx 5 / 4$, then the order is given by $4 / 5$, clearly better than the order of convergence of the uniform Euler method $(=1 / 2)$. Note that for Algorithm 4.8 , the over-all complexity of the method is, indeed, proportional to $E(N)$, with a proportionality constant only marginally larger than for the uniform Euler method. On the other hand, the complexity for Algorithm 4.6 should still be proportional to $E(N)$, but with a much larger constant.

Remark 4.18. While it is difficult to assess Assumption 4.14 by empirical observations, we have found evidence for

$$
E\left[\sum_{i=0}^{N-1} \mathbf{1}_{\left\{d\left(\bar{X}_{i}\right) \geq \delta\right\}}\right] \approx \mathrm{TOL}^{-(1+\beta) / 2}
$$

in our numerical experiments.

\section{THE ADAPTIVE ALGORITHM FOR STOPPED REFLECTED DIFFUSIONS}

Recall that the error expansion given in Proposition 3.5 can be written as a sum of three error components, coming from a purely reflecting diffusion, from a purely stopped diffusion and coming from their interplay, respectively, i.e.,

$$
\text { Error }=\text { Error }_{\text {ref }}+\text { Error }_{\text {stop }}+\text { Error }_{\text {mix }},
$$

see Remark 3.8 - notice that the stopped diffusion for Error ${ }_{\text {stop }}$ is stopped at a strange set, namely at $\partial D_{S}$. In Section 4 we have developed an adaptive algorithm for reflected dif-

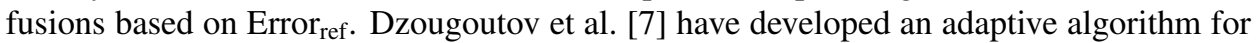

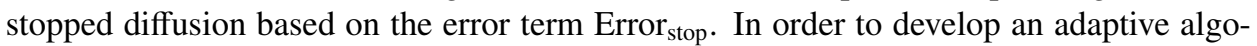
rithm for the stopped reflected diffusion, it suffices, thus, to develop an adaptive algorithm based on Error $_{\text {mix }}$. Notice, however, that

$$
\text { Error }_{\text {mix }}=E\left[\left(\partial_{t} u_{R}\left(\bar{\tau}, \bar{X}_{N_{\bar{\tau}}}\right) \Delta t_{N_{\bar{\tau}}-1}+\partial_{n} u_{R}\left(\bar{\tau}, \bar{X}_{N_{\bar{\tau}}}\right)\left\langle n\left(\bar{X}_{N_{\bar{\tau}}}\right), \bar{X}_{N_{\bar{\tau}}}-\bar{X}_{N_{\bar{\tau}}-1}\right\rangle\right) \mathbf{1}_{\{\bar{\tau}<T\}}\right]
$$

only depends on one single sub-interval, namely the sub-interval of stopping. Since this interval already contributes highly to Error $_{\text {stop }}$ (given enough regularity of the data), one might presume that this contribution is small compared to Error ref $_{\text {and Error }}$ stop. Thus, in our proposed adaptive algorithm for stopped reflected diffusions, we will ignore Error $_{\text {mix }}$ and only assume leading order term of the error of the form

$$
\text { Error }=\text { Error }_{\text {ref }}+\text { Error }_{\text {stop }} \text {. }
$$


Using the techniques of [7], this yields the following extension of Algorithm 4.6 Let $d_{R}(x)$ denote the distance to the reflecting boundary $\partial D_{R}$ and let $d_{S}(x)$ denote the distance to the stopping boundary $\partial D_{S}, x \in \bar{D}$. Following [7], we define

$$
\hat{P}_{\bar{X}, i}=P_{\bar{X}, i} \prod_{j=0}^{i-1}\left(1-P_{\bar{X}_{i}}\right), \text { with } P_{\bar{X}, i}=\exp \left(-2 \frac{d_{S}\left(\bar{X}_{i}\right) d_{S}\left(\bar{X}_{i+1}\right)}{\Delta t_{i}}\right), \quad i=0, \ldots, N-1 .
$$

The quantity $\hat{P}_{\bar{X}, i}$ serves as a rough approximation of the probability of the process $\hat{X}$ to hit the stopping boundary $\partial D_{S}$ between $t_{i}$ and $t_{i+1}$.

Remark 5.1. Following the expansion in Proposition 3.5, we should compute these quantities up to time $T$, not only up to the observed stopping time $\bar{\tau}$, since the "true", continuous stopping time may very well be after time $\bar{\tau}$ (which is not true for the purely stopped diffusion, at least if there is no interior error). However, as a second simplification, we only follow a trajectory until the first observed stopping time $\bar{\tau}$.

Moreover, we also modify the adaptive algorithm for the purely reflecting diffusion by substituting the distance to the reflecting boundary $\partial D_{R}$ for the distance to the boundary, i.e., we replace $A(x, \Delta t)$ in 4.6 by

$$
A(x, \Delta t)=-d_{R}(x)-\langle n(x), V(x)\rangle \Delta t .
$$

Algorithm 5.2 (Adaptive Euler Algorithm for Stopped Reflected Diffusions). Start with an initial mesh $\mathrm{t}$ and the corresponding Brownian motion $\bar{B}^{\mathrm{t}}$.

(1) $\operatorname{Set} \bar{X}_{0}^{\mathrm{t}}=x, \bar{Z}_{0}^{\mathrm{t}}=0, i=0$ and $N_{\bar{\tau}}=N(\mathrm{t})$.

(2) Set

$$
\hat{X}_{i+1}^{\mathrm{\dagger}}=\bar{X}_{i}^{\dagger}+V\left(\bar{X}_{i}^{\dagger}\right) \Delta t_{i}+\sum_{j=1}^{d} V_{j}\left(\bar{X}_{i}^{\dagger}\right) \Delta \bar{B}_{i}^{j}
$$

(3) Set

$$
\bar{X}_{i+1}^{\mathrm{t}}= \begin{cases}\hat{X}_{i+1}^{\mathrm{t}}, & \hat{X}_{i+1}^{\mathrm{t}} \in \bar{D} \\ \Pi\left(\hat{X}_{i+1}^{\mathrm{t}}\right), & \hat{X}_{i+1}^{\mathrm{t}} \notin \bar{D} .\end{cases}
$$

If $\bar{X}_{i+1}^{\mathrm{t}} \in \partial D_{S}$, set $N_{\bar{\tau}}=i+1$, else set

$$
\bar{Z}_{i+1}^{\mathrm{t}}= \begin{cases}\bar{Z}_{i}^{\mathrm{t}}, & \hat{X}_{i+1}^{\mathrm{t}} \in \bar{D} \\ \bar{Z}_{i}^{\mathrm{t}}+\left\|\Pi\left(\hat{X}_{i+1}^{\mathrm{t}}\right)-\hat{X}_{i+1}^{\mathrm{t}}\right\|, & \hat{X}_{i+1}^{\mathrm{t}} \notin \bar{D}\end{cases}
$$

(4) Increase $i$ by one. If $i<N(\mathrm{t})$ and $N_{\bar{\tau}}=N(\mathrm{t})$, go back to (2).

(5) Refine the grid $\mathrm{t}$ using Algorithm 5.3. If the grid has changed during refinement, then set $i=0$ and go back to (2), using the refined grid.

(6) Calculate

$$
\bar{F}^{\mathrm{t}}=f\left(\bar{X}_{N(\mathrm{t})}^{\mathrm{t}}\right) \mathbf{1}_{N_{\bar{\tau}}=N(\mathrm{t})}+g\left(\bar{X}_{N_{\bar{\tau}}}^{\mathrm{t}}\right) \mathbf{1}_{N_{\bar{\tau}}<N(\mathrm{t})}-\sum_{i=0}^{N_{\bar{\tau}}-1} h\left(\bar{X}_{i+1}^{\mathrm{t}}\right) \Delta \bar{Z}_{i}^{\mathrm{t}}
$$

Algorithm 5.3 (Refinement Algorithm). Given a time grid the Brownian motion $B^{\mathrm{t}}$ sampled on $\mathrm{t}$ and an error tolerance TOL and a stopping index $N_{\bar{\tau}}$. Do for $i=0, \ldots, N_{\bar{\tau}}-1$ :

(1) Compute a local error indicator by

$$
l_{i}=l_{i}^{(r e f)}+l_{i}^{(s t o p)},
$$

where

$$
\begin{gathered}
l_{i}^{(r e f)}=\Delta t_{i} \sigma\left(\bar{X}_{i}\right) p\left(\frac{A\left(\bar{X}_{i}, \Delta t_{i}\right)}{\sqrt{\Delta t_{i}}}\right)\left|u_{n n}\left(t_{i}, \bar{X}_{i}\right)\right| E[\widetilde{N}], \\
l_{i}^{(\text {stop })}=\left(g\left(\bar{X}_{i+1}\right)-g\left(\bar{X}_{N_{\bar{\tau}}}\right) \mathbf{1}_{N_{\bar{\tau}}<N(\mathrm{t})}-f\left(\bar{X}_{N_{\bar{\tau}}}\right) \mathbf{1}_{N_{\bar{\tau}}=N(\mathrm{t})}\right) \hat{P}_{\bar{X}, i} .
\end{gathered}
$$


(2) If $l_{i}>$ TOL, insert $t=\frac{t_{i}+t_{i+1}}{2}$ into the grid $\mathrm{t}$ and generate the corresponding sample of the Brownian motion $B_{t}$ by a Brownian bridge between the already sample values $B_{t_{i}}$ and $B_{t_{i+1}}$.

Remark 5.4. One can incorporate the explicit formula 4.11) for $\Delta t_{i}$ in the case of the purely reflecting diffusion for instance by using it to generate the initial grid in Algorithm 5.2 While this might remove a considerable part of the necessary refinements, it is possible that through the interplay of $l^{(\text {stop })}$ further refinements of the grid caused by the reflecting error contribution will be necessary. Consequently, this initialization does not allow us to set $l^{(r e f)}=0$ in Algorithm 5.3 .

Remark 5.5. In the case of a stopped reflecting diffusion, the solution $u$ usually has a singularity at points, where the different boundary conditions meet. Thus, boundedness of $u_{n n}$ is an unrealistic assumption and one should use some a-priori information of the expected singularities in these points instead. It turns out, however, that even grossly misestimated or even disregarded singularities often do not greatly reduce the efficiency of the adaptive algorithm.

\section{THE ALGORITHM WITH CORRECTION TERM FOR PURELY REFLECTING DIFFUSIONS}

Since the error term $\sqrt{3.16}$ is of order $\sqrt{\Delta t}$, it seems to be a promising idea to carry the expansion one step further. If a calculation is still feasible, we may hope to get a better order of convergence for the reflection error. Indeed, the main idea of the algorithm presented in this section is to construct a correction term such that the error expansion of the Euler algorithm with correction starts with a term of order 1, instead of order $1 / 2$ as Proposition 3.5 Of course, the computation of the correction term will introduce additional complexity into the algorithm. It will turn out, however, that the resulting algorithm is still more efficient than the ordinary Euler scheme, Algorithm 2.1. since the computation of the correction term is relatively cheap in comparison to the approximation of the reflected diffusion.

Remark 6.1. The main goal of the considerations in this section is to motivate that there seems to be no advantage in the computation of the duals weights for the adaptive algorithms presented in Section 4 This is mainly due to the extra work of computing those dual weights, which compensates possible gains in approximation accuracy. To illustrate this point, it is already sufficient to only consider the one-dimensional case, which is considerably easier for the algorithm presented in this section. Obviously, problem $[1.3$ is best solved by PDE-methods in dimension one.

Remark 6.2. The arguments presented here will stay on the heuristic level as in Remark 3.10 above. Rigorous arguments are possible using the techniques of [6].

A second order Taylor expansion of the term $(I)$ in the error expansion gives

$$
\begin{aligned}
\bar{u}(0, x)-u(0, x)= & \int_{0}^{T} E\left(\left(\bar{L}_{\bar{X}_{\lfloor t\rfloor}^{N}}-L\right) u\left(t, \bar{X}_{t}^{N}\right)\right) d t \\
& -\frac{1}{2} E\left[\sum_{i=0}^{N-1}\left(\Delta \bar{Z}_{i}^{N}\right)^{2} \frac{\partial^{2}}{\partial n\left(\bar{X}_{i+1}^{N}\right)^{2}} u\left(t_{i+1}, \bar{X}_{i+1}^{N}\right)\right] \\
& +\frac{1}{2} E\left[\sum_{i=0}^{N-1}\left(\Delta \bar{Z}_{i}^{N}\right)^{3} \times\right. \\
& \left.\times \int_{0}^{1}(1-\theta)^{2} \frac{\partial^{3}}{\partial n\left(\bar{X}_{i+1}^{N}\right)^{3}} u\left(t_{i+1}, \bar{X}_{i+1}^{N}-\theta n\left(\bar{X}_{i+1}^{N}\right) \Delta \bar{Z}_{i}^{N}\right) d \theta\right] .
\end{aligned}
$$


Consequently, let us define

$$
\overline{\bar{u}}(0, x)=E\left[f\left(\bar{X}_{N}^{N}\right)-\sum_{i=0}^{N-1} h\left(\bar{X}_{i+1}^{N}\right) \Delta \bar{Z}_{i}^{N}+\frac{1}{2} \sum_{i=0}^{N-1}\left(\Delta \bar{Z}_{i}^{N}\right)^{2} \overline{\partial_{n}^{2} u}\left(t_{i+1}, \bar{X}_{i+1}^{N}\right)\right],
$$

where $\overline{\partial_{n}^{2} u}\left(t_{i+1}, \bar{X}_{i+1}^{N}\right)$ is a computable approximation of

$$
\overline{\partial_{n}^{2} u}\left(t_{i+1}, \bar{X}_{i+1}^{N}\right) \approx \frac{\partial^{2}}{\partial n\left(\bar{X}_{i+1}^{N}\right)^{2}} u\left(t_{i+1}, \bar{X}_{i+1}^{N}\right) .
$$

Remark 6.3. If we could work with the exact value for the second normal derivative, then equation 6.1 would give a precise error expansion for $\overline{\bar{u}}$. Indeed, with the same heuristics as before, this approximate error expansion indicates a convergence of order 1 . Of course, this presupposes that the approximation $\overline{\partial_{n}^{2} u}$ can be calculated with sufficient accuracy and efficiency.

We present a scheme for efficient computation of $\overline{\partial_{n}^{2} u}$ applicable in dimension one. Unfortunately, we have not found sufficiently efficient methods to approximate the second normal derivative of $u$ in more generality, but under special circumstances, see the discussion at the end of this section. For the remainder of the section, we assume $d=1$. Consequently, all the normal derivatives are, in fact, ordinary derivatives with respect to the space variable $x$, possibly with a sign, and the domain is an interval $D=] a, b$, where one of $a, b \in \mathbb{R} \cup\{ \pm \infty\}$ may be $\pm \infty$. More precisely, the Neumann boundary condition reads

$$
\frac{\partial}{\partial x} u(t, a)=h(a), \quad \frac{\partial}{\partial x} u(t, b)=-h(b),
$$

provided that both $a$ and $b$ are real numbers. For simplicity, we will only consider the case $a=0$ and $b=+\infty$, the extension to the general case is trivial. $u_{x}=\frac{\partial}{\partial x} u$ solves the following PDE with Dirichlet boundary conditions

$$
\left\{\begin{aligned}
\frac{\partial}{\partial t} u_{x}(t, x)+\widetilde{L} u_{x}(t, x) & =0, \quad(t, x) \in[0, T[\times D, \\
u_{x}(T, x) & =f^{\prime}(x), \quad x \in \bar{D}, \\
u_{x}(t, x) & =h(x), \quad(t, x) \in[0, T[\times \partial D .
\end{aligned}\right.
$$

In (6.4), the differential operator $\widetilde{L}$ is no longer of the same form as $L$, but it still allows for a stochastic representation using the Feynman-Kac formula. Indeed, if

$$
L g(x)=V(x) g^{\prime}(x)+\frac{1}{2}\left(V_{1}(x)\right)^{2} g^{\prime \prime}(x)
$$

then the solution of the heat equation with

$$
\widetilde{L} g(x)=V^{\prime}(x) g(x)+\left(V(x)+V_{1}^{\prime}(x)\right) g^{\prime}(x)+\frac{1}{2}\left(V_{1}(x)\right)^{2} g^{\prime \prime}(x)
$$

has the stochastic representation

$$
\begin{aligned}
u_{x}(t, x)= & E\left[f^{\prime}\left(X_{T}\right) \exp \left(\int_{t}^{T} V^{\prime}\left(X_{s}\right) d s\right) \mathbf{1}_{[T, \infty[}\left(\tau_{t, x}\right) \mid X_{t}=x\right] \\
& +E\left[h\left(X_{\tau_{t, x}}\right) \exp \left(\int_{t}^{\tau_{t, x}} V^{\prime}\left(X_{s}\right) d s\right) \mathbf{1}_{[t, T[}\left(\tau_{t, x}\right) \mid X_{t}=x\right],
\end{aligned}
$$

where

$$
\tau_{t, x}=\inf \left\{s>t \mid X_{s}^{t, x} \in \partial D\right\},
$$

see (6.6). Consequently, in order to compute $u_{x}(t, x)$ one needs to solve a stopped diffusion following the well known SDE

$$
d X_{s}^{t, x}=V\left(X_{s}^{t, x}\right) d s+V_{1}\left(X_{s}^{t, x}\right) d B_{s}^{1}, \quad s>t,
$$


started at time $t$ and $X_{t}^{t, x}=x$ and stopped when hitting the boundary $\partial D$.

Remark 6.4. Notice that stopped diffusions are much simpler problems than reflected diffusions. As already indicated in the beginning of this subsection, the computation of the correction term amounts to additionally solving a number of simple problems (compared to the reflected diffusion), namely the stopped diffusions, in order to obtain a faster convergence rate for the reflected diffusion.

The idea for the approximation $\overline{\partial_{n}^{2} u}$ is the following. First note that $\overline{\partial_{n}^{2} u}(t, x)$ is only needed for $x \in \partial D$, i.e., $x=0$, because otherwise $\Delta \bar{Z}=0$ owing Algorithm 2.1, see (6.2). We approximate $u_{x x}(t, 0)=\frac{\partial^{2}}{\partial x^{2}} u(t, x)$ by a finite difference quotient, i.e.,

$$
\begin{aligned}
u_{x x}(t, 0) & =-\frac{u_{x}(t, 0)-u_{x}(t, \Delta x)}{\Delta x}+O(\Delta x) \\
& =-\frac{h(0)-u_{x}(t, \Delta x)}{\Delta x}+O(\Delta x),
\end{aligned}
$$

for $\Delta x>0$ sufficiently small. $u_{x}(t, \Delta x)$ is then approximated by a stopped diffusion. Notice that we do not need to perform an "inner" Monte Carlo simulation for this computation. Indeed, let $G(t, x)$ denote the random variable under the expectation in 6.5, i.e.,

$$
\begin{aligned}
G(t, x)=f^{\prime}\left(X_{T}^{t, x}\right) \exp \left(\int_{t}^{T} V^{\prime}\left(X_{s}^{t, x}\right) d s\right) \mathbf{1}_{[T, \infty[}\left(\tau_{t, x}\right) & \\
& +h\left(X_{\tau_{t, x}^{t, x}}^{t, x}\right) \exp \left(\int_{t}^{\tau_{t, x}} V^{\prime}\left(X_{s}^{t, x}\right) d s\right) \mathbf{1}_{[t, T[}\left(\tau_{t, x}\right) .
\end{aligned}
$$

Notice that $G(t, x)$ is independent of $\mathcal{F}_{t}$. Consequently, we have

$$
\begin{aligned}
E\left[\sum_{i=0}^{N-1} u_{x x}\left(t_{i+1}, \bar{X}_{i+1}^{N}\right)\left(\Delta \bar{Z}_{i}^{N}\right)^{2}\right] & \approx E\left[\sum_{i=0}^{N-1} \frac{u_{x}\left(t_{i+1}, \Delta x\right)-h(0)}{\Delta x}\left(\Delta \bar{Z}_{i}^{N}\right)^{2}\right] \\
& =E\left[\sum_{i=0}^{N-1} \frac{E\left(G\left(t_{i+1}, \Delta x\right)\right)-h(0)}{\Delta x}\left(\Delta \bar{Z}_{i}^{N}\right)^{2}\right] \\
& =E\left[\sum_{i=0}^{N-1} \frac{G\left(t_{i+1}, \Delta x\right)-h(0)}{\Delta x}\left(\Delta \bar{Z}_{i}^{N}\right)^{2}\right],
\end{aligned}
$$

by independence of $G\left(t_{i+1}, \Delta x\right)$ and $\Delta \bar{Z}_{i}^{N}$. In order to get a workable algorithm, we need to fix an approximation $\bar{G}(t, \Delta x)$ of $G(t, \Delta x)$. Then, we can finally set

$$
\overline{\partial_{n}^{2} u}\left(t_{I+1}, \bar{X}_{i+1}^{N}\right)=\frac{\bar{G}\left(t_{i+1}, \Delta x\right)-h(0)}{\Delta x} .
$$

Remark 6.5. Of course, $\overline{\partial_{n}^{2} u}$ as in 6.8 is not a true approximation of $u_{x x}$, only its expected value is. $\Delta x$ is a critical parameter for the algorithm and needs to be chosen depending on $N$ and on the approximation method $\bar{G}$.

We use two different methods $\bar{G}$, namely the uniform Euler method, see Gobet [12], and an adaptive Euler scheme, see Dzougoutov et al. [7]. It is well known that the uniform Euler scheme converges with the rate $1 / 2$, whereas the adaptive scheme has the (conjectured) order 1 .

Let us first consider the uniform scheme, i.e., let $\left(\bar{X}_{j}^{N, t_{i+1}, \Delta x}\right)_{j=i+1}^{N}$ be the Euler approximation of the SDE started at $\bar{X}_{i+1}^{N, t_{i+1}, \Delta x}=\Delta x$ and calculated along the uniform grid $t_{i+1}<$ $t_{i+2}<\ldots<t_{N}=T$. More precisely, we have

$$
\bar{X}_{j+1}^{N, t_{i+1}, \Delta x}=\bar{X}_{j}^{N, t_{i+1}, \Delta x}+V\left(\bar{X}_{j}^{N, t_{i+1}, \Delta x}\right) \Delta t_{j}+V_{1}\left(\bar{X}_{j}^{N, t_{i+1}, \Delta x}\right) \Delta B_{j}^{1},
$$


$j=i+1, \ldots, N-1$. Moreover, let $\bar{\tau}^{N, t_{i+1}, \Delta x}$ be the first hitting time of the discrete process $\bar{X}^{N, t_{i+1}, \Delta x}$ at $D^{c}-$ we set $\bar{\tau}^{N, t_{i+1}, \Delta x}=\infty$ if no hitting occurs. For $t_{k+1} \geq t_{i+1}$ let

$$
I\left(t_{i+1}, t_{k+1}\right)=\exp \left(\sum_{j=i+1}^{k} V^{\prime}\left(\bar{X}_{j+1}^{N, t_{i+1}, \Delta x}\right) \Delta t_{j}\right)
$$

and define

$$
\begin{aligned}
\bar{G}^{u n}\left(t_{i+1}, \Delta x\right)=\left(f^{\prime}\left(\bar{X}_{N}^{N, t_{i+1}, \Delta x}\right) \mathbf{1}_{\{T, \infty\}}\left(\bar{\tau}^{N, t_{i+1}, \Delta x}\right)\right. \\
\left.+h\left(\bar{X}_{\bar{\tau}^{N, t_{i+1}, \Delta x}}^{N, t_{i+1}, \Delta x}\right) \mathbf{1}_{\left\{t_{i+2}, \ldots, t_{N-1}\right\}}\left(\bar{\tau}^{N, t_{i+1}, \Delta x}\right)\right) I\left(t_{i+1}, \bar{\tau}^{N, t_{i+1}, \Delta x}\right) .
\end{aligned}
$$

Of course, if the boundary has been hit before $j=N, \bar{G}^{\text {un }}$ can already be computed and the iteration does not need to be continued until time $T$.

Remark 6.6. Since we solve the stopped diffusion with the same grid as the outer reflected diffusion, we do not need to sample additional Brownian increments.

We still have to choose the parameter $\Delta x$. Notice that we approximate the second derivative $u_{x x}$ with the error

$$
u_{x x}(t, 0)=\frac{E(\bar{G}(t, \Delta x))-h(0)}{\Delta x}+\frac{O(\sqrt{\Delta t})}{\Delta x}+O(\Delta x),
$$

where $\Delta t=T / N$. Optimizing the error with respect to $\Delta x$ yields

$$
\Delta x=\text { const } \times(\Delta t)^{1 / 4}=\text { const } \times\left(\frac{T}{N}\right)^{1 / 4} .
$$

This gives an error from the approximation of the correction term of order

$$
E\left[\sum_{i=0}^{N-1} O\left((\Delta t)^{1 / 4}\right)\left(\Delta \bar{Z}_{i}^{N}\right)^{2}\right]=O(\sqrt{N}) \times O\left(N^{-1 / 4}\right) \times O\left(N^{-1}\right)=O\left(N^{-3 / 4}\right) .
$$

However, we have an additional complexity due to the simulation of $\bar{G}$. Indeed, since $E\left(\bar{\tau}^{N, t, \Delta x}\right)=O(\Delta x)$, the additional work is of order $N \times \frac{O(\Delta x)}{T}=O\left(N^{3 / 4}\right)$ per reflection at the boundary. Since these reflections take place $O(\sqrt{N})$ times, the additional and, hence, the total work is $O\left(N^{5 / 4}\right)$. Therefore, the rate of convergence with respect to the total work $K \approx$ const $\times N^{5 / 4}$ is expected to be $O\left(K^{-3 / 5}\right)$.

Remark 6.7. Of course, all the arguments in the preceding paragraph are highly heuristic. For example, the number of hits at the boundary (of the reflected trajectory) and the time until stopping (of the stopped trajectory using the same Brownian increments) are certainly not independent. However, independence would hold if we sampled new, independent increments of Brownian motion for the stopped diffusion. Nevertheless, all the above observations seem to be consistent with the results of numerical experiments.

Remark 6.8. $E\left(\bar{\tau}^{N, t, \Delta x}\right)=O(\Delta x)$ is motivated by the following consideration. Let $B_{t}^{\Delta x}$ be a 1-dimensional Brownian motion started at $B_{0}^{\Delta x}=\Delta x>0$ and denote by $\tau^{\Delta x}$ the first hitting time of $B_{t}^{\Delta x}$ at 0 , if $B_{t}^{\Delta x}$ hits 0 before time $T$, and $\tau^{\Delta x}=T$ otherwise. The distribution of $\tau^{\Delta x}$ is given by

$$
\begin{aligned}
& P\left(\tau^{\Delta x} \in d s\right)=\frac{\Delta x}{\sqrt{2 \pi s^{3}}} e^{-\frac{\Delta x^{2}}{2 s}} d s, \quad s \in[0, T[, \\
& P\left(\tau^{\Delta x}=T\right)=\frac{2}{2 \pi} \int_{0}^{\Delta x / \sqrt{T}} e^{-\frac{s^{2}}{2}} d s,
\end{aligned}
$$


see Karatzas and Shreve [14]. The first two moments of $\tau^{\Delta x}$ can be explicitly calculated and an expansion in $\Delta x$ gives

$$
E\left(\tau^{\Delta x}\right)=\frac{2 \sqrt{2 T}}{\sqrt{\pi}} \Delta x+O\left(\Delta x^{2}\right) .
$$

We do not give a detailed description of the adaptive algorithm for stopped diffusions, see Section 5 for some hints. We just mention that the adaptive algorithm has an empirical rate of convergence 1 in the general case. If the coefficients of the equation are constant, i.e., if there is no error stemming from the discretization in the interior of the domain, then the adaptive algorithm for the stopped diffusion problem converges exponentially fast. Therefore, we can now approximate the second derivative $u_{x x}$ with an error

$$
u_{x x}(t, 0)=\frac{E\left(\bar{G}^{a d}(t, \Delta x)\right)-h(0)}{\Delta x}+\frac{O(\Delta t)}{\Delta x}+O(\Delta x),
$$

where $\bar{G}^{a d}(t, \Delta x)$ denotes the stopped diffusion started at $\Delta x$ computed with the adaptive algorithm (noting that the discretization grid is random, too). Minimizing the error gives

$$
\Delta x=\text { const } \times \sqrt{\Delta t}=\text { const } \times \sqrt{\frac{T}{N}} .
$$

Consequently, the error from the approximation in the correction term is this time

$$
E\left[\sum_{i=0}^{N-1} O(\sqrt{\Delta t})\left(\Delta \bar{Z}_{i}^{N}\right)^{2}\right]=O(\sqrt{N}) \times O(1 / \sqrt{N}) \times O(1 / N)=O(1 / N)
$$

However, note that we still have an additional work of order $O\left(N^{5 / 4}\right)$, which leads to the total convergence rate $O\left(K^{-4 / 5}\right)$. Notice that this is the same rate as empirically found for the adaptive algorithm. Only in case of constant coefficients, the additional work is still of $O(N)$, therefore giving us the desired order $O\left(N^{-1}\right)$.

Concluding, we propose the following algorithm for one dimensional problems.

Algorithm 6.9. Fix a uniform time discretization $0=t_{0}<t_{1}<\cdots<t_{N}=T$, i.e., $\Delta t_{i}=t_{i+1}-t_{i}=\frac{T}{N}, i=0, \ldots, N-1$. Moreover, fix an i. $i$. $d$. sequence of random variables $\left(\Delta \bar{B}_{i}\right)_{i=0}^{N-1}$ such that the moments of order up to three of $\Delta \bar{B}_{0}$ (and hence of all $\Delta \bar{B}_{i}$ ) coincide with those of an one dimensional normal random variable with variance $\frac{T}{N}$.

(1) $\operatorname{Set} \bar{X}_{0}^{N}=x, \bar{Z}_{0}^{N}=0$, set $i=0$.

(2) Set

$$
\hat{X}_{i+1}^{N}=\bar{X}_{i}^{N}+V\left(\bar{X}_{i}^{N}\right) \Delta t_{i}+\sum_{j=1}^{d} V_{j}\left(\bar{X}_{i}^{N}\right) \Delta \bar{B}_{i}^{j}
$$

(3) Set

$$
\begin{aligned}
& \bar{X}_{i+1}^{N}= \begin{cases}\hat{X}_{i+1}^{N}, & \hat{X}_{i+1}^{N} \in \bar{D}, \\
\Pi\left(\hat{X}_{i+1}^{N}\right), & \hat{X}_{i+1}^{N} \notin \bar{D},\end{cases} \\
& \bar{Z}_{i+1}^{N}= \begin{cases}\bar{Z}_{i}^{N}, & \hat{X}_{i+1}^{N} \in \bar{D}, \\
\bar{Z}_{i}^{N}+\left\|\Pi\left(\hat{X}_{i+1}^{N}\right)-\hat{X}_{i+1}^{N}\right\|, & \hat{X}_{i+1}^{N} \notin \bar{D} .\end{cases}
\end{aligned}
$$

(4) If $\Delta \bar{Z}_{i}^{N}>0$, calculate either $\bar{G}^{\text {un }}\left(t_{i+1}, \Delta t^{1 / 4}\right)$ or $\bar{G}^{\text {ad }}\left(t_{i+1}, \sqrt{\Delta t}\right)$ and compute the approximate second order normal derivative $\overline{\partial_{n}^{2} u}\left(t_{i+1}, 0\right)$ according to 6.8 .

(5) Increase $i$ by one. If $i<N$, go back to (2).

(6) Calculate

$$
\bar{F}^{N}=f\left(\bar{X}_{N}^{N}\right)-\sum_{i=0}^{N-1} h\left(\bar{X}_{i+1}^{N}\right) \Delta \bar{Z}_{i}^{N}+\frac{1}{2} \sum_{i=0}^{N-1}\left(\Delta \bar{Z}_{i}^{N}\right)^{2} \overline{\partial_{n}^{2} u}\left(t_{i+1}, \bar{X}_{i+1}^{N}\right) .
$$


Remark 6.10. In the $d$-dimensional situation with $d>1$, we can use the same finite difference approximation as 6.7, i. e.

$$
u_{n n}(t, x)=\frac{u_{n}(t, x+\Delta x n(x))-h(x)}{\Delta x}+O(\Delta x),
$$

where $u_{n}$ and $u_{n n}$ are the needed normal derivatives, $x \in \partial D$. Unless $n(x)$ is a constant vector (corresponding to a half-space domain), we do not get a PDE for $u_{n}$. However, using the stochastic representation of the original problem, we get

$$
u_{n}(t, x+\Delta x n(x))=E\left[\left\langle\nabla u\left(\tau, X_{\tau}^{t, x+\Delta x n(x)}\right), J_{t \rightarrow \tau}(x+\Delta x n(x)) n(x)\right\rangle\right],
$$

where $X^{t, x+\Delta x n(x)}$ denotes the solution of the SDE started at time $t$ with $X_{t}^{t, x+\Delta x n(x)}=x+$ $\Delta x n(x)$ and $\tau$ denotes the first hitting time of $X^{t, x+\Delta x n(x)}$ at $\partial D$ (or $\tau=T$ if no such hit occurs before $T) . J_{t \rightarrow s}(x+\Delta x n(x))$ is the first variation, i.e., the (path-wise) Jacobi matrix of the map $y \mapsto X_{s}^{t, y}$ evaluated at $y=x+\Delta x n(x)$. By the boundary condition,

$$
\left\langle\nabla u\left(\tau, X_{\tau}^{t, x+\Delta x n(x)}\right), n\left(X_{\tau}^{t, x+\Delta x n(x)}\right)\right\rangle=h\left(X_{\tau}^{t, x+\Delta x n(x)}\right),
$$

but

$$
J_{t \rightarrow \tau}(x+\Delta x n(x)) n(x) \neq n\left(X_{\tau}^{t, x+\Delta x n(x)}\right)
$$

in general. Nevertheless, we might boldly use

$$
u_{n}(t, x+\Delta x n(x)) \approx E\left(h\left(X_{\tau}^{t, x+\Delta x n(x)}\right)\right),
$$

but that approximation would lead to a non-converging scheme unless the "normal vector field" $n(x)$ can be extended to $x \in D$ and commutes with $L$.

\section{NUMERICAL EXAMPLES}

We illustrate the performance of the algorithms presented in the previous sections by some numerical examples in dimension one and two. For reporting the results of the computations, we use the following nomenclature. By "error" we understand the computational error in the sense of

$$
\text { Error }=\bar{u}\left(t_{0}, x_{0}\right)-u\left(t_{0}, x_{0}\right)
$$

where $\bar{u}\left(t_{0}, x_{0}\right)$ denotes the computed approximation to the true value $u\left(t_{0}, x_{0}\right)$ of the respective problem. We denote the number of paths used in the Monte-Carlo simulation by $M$. An estimate for the possible "statistical" error component caused by the computation of the expected value of the random variable $\bar{F}^{N}$ constructed by the relevant numerical scheme by Monte-Carlo simulation is given by $\mathcal{S}$. Indeed, let $\bar{F}_{i}^{N}$ denote the $i$ th sample of $\bar{F}^{N}$, $i=1, \ldots, M$. Ideally, these samples are mutually independent and identically distributed, which allows us to appeal to the central limit theorem, giving

$$
\sqrt{M}\left(E\left(\bar{F}^{N}\right)-\frac{1}{M} \sum_{i=1}^{M} \bar{F}_{i}^{N}\right) \underset{M \rightarrow \infty}{\longrightarrow} \mathcal{N}\left(0, \sigma^{2}\right),
$$

where the convergence is understood as convergence in distribution and $\sigma^{2}$ denotes the variance of $\bar{F}^{N}$. Heuristically, we may assume that $M$ is already large enough that the convergence in the central limit theorem has already taken place, i.e.,

$$
\text { Error } \approx \mathcal{N}\left(0, \frac{\bar{\sigma}^{2}}{M}\right)
$$

where $\bar{\sigma}^{2}$ denotes the empirical variance of the sample $\left(\bar{F}_{i}^{N}\right)_{i=1}^{M}$. This gives us a $90 \%$ confidence interval,

$$
E\left(\bar{F}^{N}\right)-u\left(t_{0}, x_{0}\right) \in\left[\text { Error }-1.65 \times \frac{\bar{\sigma}}{\sqrt{M}}, \text { Error }+1.65 \times \frac{\bar{\sigma}}{\sqrt{M}}\right] .
$$


We denote

$$
\mathcal{S}=1.65 \times \frac{\bar{\sigma}}{\sqrt{M}}
$$

and report it in Table 1 and all the subsequent tables.

Remark 7.1. The quantity $\mathcal{S}$ as defined in (7.2) is an indicator for the size of the statistical error, possibly overlapping the error from the time discretization in the observed computational error. Recall that the computational error can be decomposed as

$$
u\left(t_{0}, x_{0}\right)-\underbrace{\frac{1}{M} \sum_{i=1}^{M} \bar{F}_{i}^{N}}_{=\bar{u}\left(t_{0}, x_{0}\right)}=\underbrace{u\left(t_{0}, x_{0}\right)-E\left[\bar{F}^{N}\right]}_{=\mathrm{E}_{\mathrm{disc}}}+\underbrace{E\left[\bar{F}^{N}\right]-\frac{1}{M} \sum_{i=1}^{M} \bar{F}_{i}^{N}}_{=\mathrm{E}_{\text {stat }}} .
$$

$\mathrm{E}_{\text {disc }}$ can be naturally interpreted as the error caused by the time-discretization of the dynamics of the (reflected) SDE, whereas $E_{\text {stat }}$ is the "statistical" error caused by Monte-Carlo simulation, i.e., the integration error. In this study, we are only interested in the discretization error $\mathrm{E}_{\mathrm{disc}}$, since we want to analyze the behavior of special time-discretization algorithms. Consequently, we need to make sure that our results are not overshadowed by the statistical error. In practice, we choose $M$ large enough such that $\mathcal{S}=\mathcal{S}(M)$ is much smaller than the observed computational error. For a thorough treatment of the types of errors involved in Euler Monte-Carlo schemes, we refer to [16] and [20], and to Glasserman [11] for general application of Monte-Carlo methods.

We would like to stress that the statistical error is, in practice, a very important part of the overall computational error. Therefore, the strategy advocated above is not suited for true computations, but only for the purpose of analysis of algorithms as mentioned above.

For the adaptive algorithms presented in Section 4 and Section 5 we still need to specify one parameter, namely the expected number of hits at the reflecting boundary $\partial D_{R}, E[\widetilde{N}]$. Note that $E[\widetilde{N}]$ as a parameter in the adaptive algorithm influences the number of hits at the boundary obtained by the same algorithm. Therefore, we need to choose $E[\widetilde{N}]$ in such a way that the parameter for the adaptive algorithm is consistent in the sense that the average number of hits of the adaptive algorithm coincides with the parameter. Since we have not found a formula for $E[\widetilde{N}]$, we are left with two possibilities:

(1) We can start with some initial guess $N_{0}$ for $E[\widetilde{N}]$ and run the adaptive algorithm with it. Taking the average of the number of hits at the reflecting boundary, we obtain an estimate $N_{1}$ for the expected number of hits of the boundary for the adaptive algorithm run with $N_{0}$. If $N_{0} \approx N_{1}$, we run the adaptive algorithm with $N_{1}$, thus obtaining an estimate $N_{2}$. We iterate this procedure until we find $N_{k} \approx$ $N_{k+1}$, and use this number as parameter for the adaptive algorithm for the problem at hand.

(2) We make the Ansatz $E[\widetilde{N}]=\mathrm{TOL}^{-\beta}$ for some $\beta>0$ and estimate $\beta$ for various model problems. Of course, the same problem as above applies, i.e., $\beta$ must be consistent in the sense that using $E[\widetilde{N}] \approx \mathrm{TOL}^{-\beta}$ in the algorithm must give an empirical mean number of hits $\approx \mathrm{TOL}^{-\beta}$. It turns out that $\beta$ seems to be easy to estimate, and we get consistent results in the range $\beta \in[1.15,1.25]$ for all our numerical tests. Thus we think that $\beta$ is a largely problem-independent quantity. When we draw conclusions from $\beta$, we will stick to the least favorable value $\beta=$ $1.25=5 / 4$.

7.1. A reflected diffusion in dimension 1. We start with a one-dimensional example, which mainly serves as illustration of the algorithm with correction, cf. Algorithm 6.9 Because we are only interested in the error coming from the reflection at the boundary, 
we use a problem without interior discretization error, i.e., in the interior of the domain $D=[0, \infty[$. The solution process $X$ is the driving Brownian motion $B$ reflected at the boundary $\partial D=\{0\}$. The Neumann boundary condition and the initial condition are fixed as follows:

$$
\left\{\begin{aligned}
\frac{\partial}{\partial t} u(t, x) & =-\frac{1}{2} \Delta u(t, x), \quad(t, x) \in[0,2] \times[0, \infty[ \\
u(2, x) & =e^{2}(\sin (\sqrt{2} x)+\cos (\sqrt{2} x)), \quad x \in[0, \infty[ \\
\frac{\partial}{\partial x} u(t, 0) & =\sqrt{2} e^{t}, \quad t \in[0,2] .
\end{aligned}\right.
$$

Obviously, problem 7.3 has the explicit solution

$$
u(t, x)=e^{t}(\sin (\sqrt{2} x)+\cos (\sqrt{2} x))
$$

Remark 7.2. In [6], the authors prove that the uniform Euler algorithm for the Brownian motion reflected at 0 (but with different boundary and initial conditions) does not converge faster than with rate one half. We have changed the boundary conditions because the third derivative of the solution of their problem vanishes, which would lead to untypically fast convergence rates for the algorithm with second order correction terms, because its leading order error term involves the third derivative. As can be easily checked, the third derivative of (7.4) does not vanish for $x=0$.

We compute the value $u\left(0, x_{0}\right)$ for $x_{0}=0.5$ using three different methods: the uniform Euler method, cf. Algorithm 2.1, the uniform Euler method with second order correction term computed using a stopped diffusion approximated by the adaptive algorithm of [7], cf. Algorithm 6.9 Finally, we also compute the outcome with the adaptive Algorithm 4.6 Notice that the exact value is $u(0,0.5) \approx 1.4099$.

\begin{tabular}{|r|r|c|c|}
\hline \multicolumn{1}{|c|}{$N$} & $M$ & Error & $\mathcal{S}$ \\
\hline 2 & 80080 & 0.3811 & 0.0416 \\
4 & 80320 & 0.5986 & 0.0386 \\
8 & 56400 & 0.6252 & 0.0448 \\
16 & 62800 & 0.5417 & 0.0420 \\
32 & 75600 & 0.4493 & 0.0382 \\
64 & 101200 & 0.3581 & 0.0329 \\
128 & 152400 & 0.2461 & 0.0269 \\
256 & 254800 & 0.1822 & 0.0208 \\
512 & 459600 & 0.1330 & 0.0155 \\
1024 & 869200 & 0.0881 & 0.0113 \\
2048 & 1688400 & 0.0638 & 0.0081 \\
4096 & 3326800 & 0.0496 & 0.0058 \\
\hline
\end{tabular}

Table 1. Results of the uniform Euler Monte-Carlo algorithm for problem 7.3

Table 1 gives the results for the uniform algorithm. The results show the typical convergence order $1 / 2$ of the reflected uniform Euler method. See also Figure 2 for comparisons of the different algorithms used.

Table 2 shows the results for the algorithm with second order correction term, cf. Algorithm 6.9. The correction term $\bar{G}^{\text {ad }}$ is calculated using the adaptive Euler algorithm for stopped diffusions presented in [7]. The local error tolerance parameter is chosen to be

$$
\mathrm{TOL}=\frac{1}{\log (N)},
$$




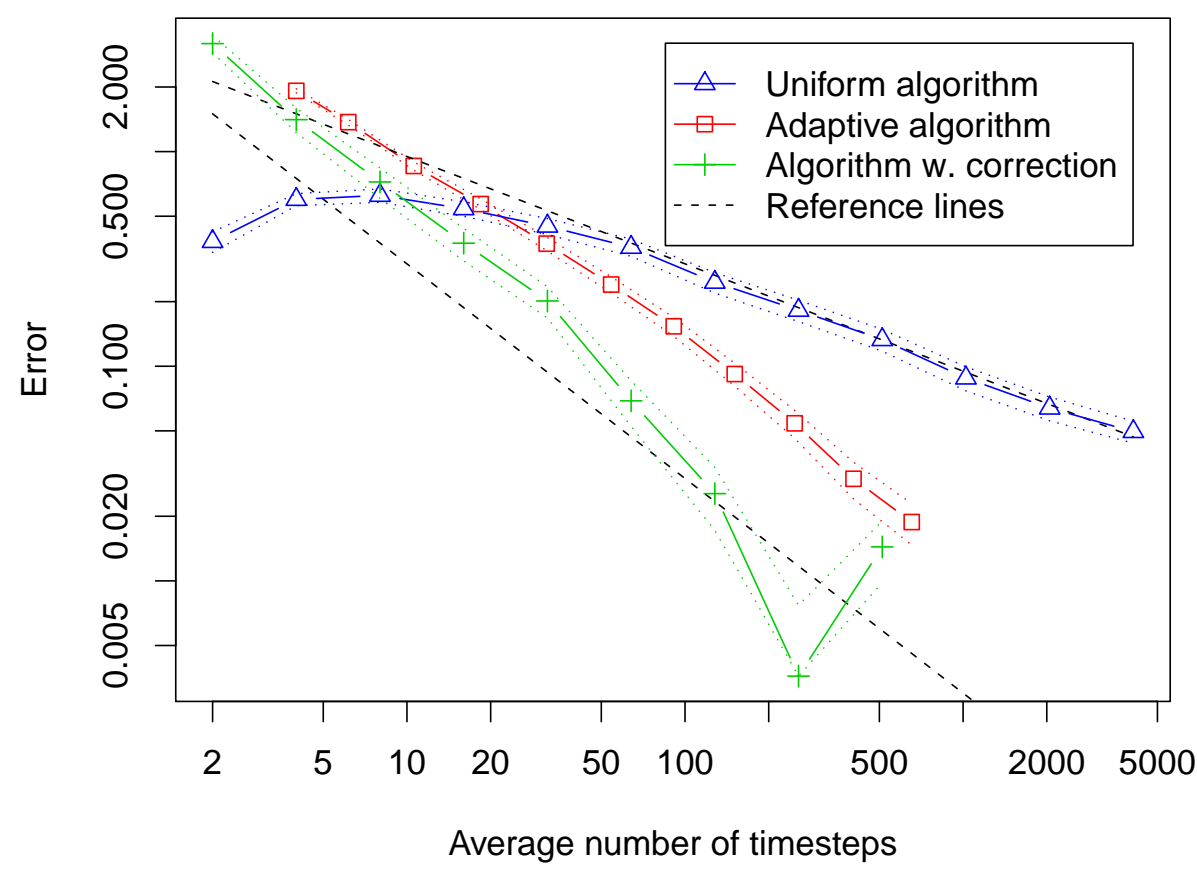

Figure 2. Absolute value of the computational error for problem 7.3 . The dotted lines show confidence areas for the true error in the sense of equation (7.1) and the dashed lines in black are reference lines of order $1 / N$ and $1 / \sqrt{N}$, respectively. Notice that the "Average number of time-steps" is understood as the work in the above sense in the case of the algorithm with a second order correction term and as average size of the refined mesh in the case of the adaptive algorithm. The dashed line for the adaptive algorithm corresponds to the error estimate computed for the refinement algorithm and is clearly proportional to the observed computational error.

\begin{tabular}{|r|r|r|c|c|}
\hline \multicolumn{1}{|c|}{$N$} & \multicolumn{1}{|c|}{ Work } & \multicolumn{1}{|c|}{$M$} & Error & $\mathcal{S}$ \\
\hline 2 & 2.45 & 5400 & -3.1856 & 0.2928 \\
4 & 7.74 & 6600 & -1.4092 & 0.1838 \\
8 & 19.35 & 11400 & -0.7224 & 0.1163 \\
16 & 43.66 & 30600 & -0.3743 & 0.0647 \\
32 & 92.22 & 107400 & -0.2014 & 0.0333 \\
64 & 193.13 & 414600 & -0.0690 & 0.0166 \\
128 & 401.43 & 1643400 & -0.0255 & 0.0083 \\
256 & 831.52 & 6558600 & -0.0036 & 0.0041 \\
512 & 3065.85 & 5262880 & -0.0144 & 0.0046 \\
\hline
\end{tabular}

Table 2. Results of the uniform Euler Monte-Carlo algorithm with second order correction term calculated using an adaptive algorithm for problem (7.3).

which is consistent with the observation that the error decreases exponentially fast, implying an approximation error proportional to $\frac{1}{N}$ for the correction term using the above local error tolerance. 
As before, $N$ is the size of the uniform base grid of the discretization of the reflected diffusion. Work denotes the average work per realization, i.e., for one single realization, the corresponding work is $N$ plus the total work for each stopped diffusion, which is computed in order to get the respective correction terms.

The results of the algorithm with correction show an empirical order of convergence one, i.e., like $1 / N$, even if the total work in the above sense is used as reference. As remarked above Algorithm 6.9, this is only possible since the approximation is precise in the interior of the domain. Compare also Figure 2, which shows the superiority of the algorithm with correction over the other two proposed algorithms. (Notice that the confidence interval for the last but second result of this algorithm could not be plotted in Figure 2, because it contains negative numbers. Therefore, this confidence interval was changed manually.)

Finally, Table 3 shows the results of the adaptive Algorithm 4.6 applied to problem 7.3 .

\begin{tabular}{|c|r|r|c|c|r|}
\hline TOL & \multicolumn{1}{|c|}{$N$} & \multicolumn{1}{c|}{$M$} & Error & $\mathcal{S}$ & \multicolumn{1}{|c|}{$\widetilde{N}$} \\
\hline 0.5743 & 4 & 56400 & 1.9191 & 0.0424 & 0.79 \\
0.3789 & 6.15 & 62800 & 1.3719 & 0.0409 & 1.36 \\
0.2500 & 10.58 & 75600 & 0.8574 & 0.0377 & 2.31 \\
0.1649 & 18.41 & 101200 & 0.5698 & 0.0328 & 3.90 \\
0.1088 & 31.81 & 152400 & 0.3725 & 0.0268 & 6.45 \\
0.0718 & 54.25 & 254800 & 0.2402 & 0.0208 & 10.59 \\
0.0474 & 91.07 & 459600 & 0.1535 & 0.0155 & 17.23 \\
0.0313 & 150.94 & 869200 & 0.0920 & 0.0113 & 27.83 \\
0.0206 & 247.98 & 1688400 & 0.0542 & 0.0081 & 44.83 \\
0.0136 & 403.61 & 3326800 & 0.0299 & 0.0058 & 71.93 \\
0.0090 & 653.62 & 6603600 & 0.0188 & 0.0041 & 115.28 \\
\hline
\end{tabular}

Table 3. Results of the adaptive Euler Monte-Carlo algorithm for problem (7.3).

Here, TOL is the local error tolerance used for the adaptive algorithm, cf. Algorithm 4.7 The second order normal derivative in the local error term 4.10 ) is approximated by the constant 1 . The number of steps in the uniform initial grid is always 2 , which is, of course, unrealistically small but probably useful for the theoretical understanding of the refinements. The value $N$ as reported in the second column of Table 3 corresponds to the average size of the final mesh, we use Algorithm 4.8 . Finally, $\widetilde{N}$ as reported in Table 3 is the average number of hits at the boundary.

The observed computational error of the adaptive algorithm lies between the errors for the uniform Euler algorithms with and without correction term, as expected. A fit of the observed average number of hits at the boundary gives $E[\widetilde{N}] \approx \mathrm{TOL}^{-1.19}$, reasonably close to the dependence $\mathrm{TOL}^{-\beta}$ with $\beta=1.25$ used in the algorithm. While $\beta=1.25$ would correspond to a convergence like $N^{-0.8}$, a fit of the error in terms of the size of the adaptively generated mesh gives Error $\approx E[N]^{-0.89}$. If we can choose $\beta$ even smaller than 1.25 , as indicated by the results, then the theoretical rate of convergence is improved.

In the tables above we have noted certain indicators for the computational cost of running the various algorithms. While these numbers are useful in showing the dependence of the computational cost for one particular algorithm depending on the grid size, the tolerance or both, it does not allow to compare the efficiency of different algorithms other than in an asymptotic sense. Note that it is easy to compare the uniform Euler scheme with the adaptive Euler scheme in its explicit form, see Algorithm 4.8 Indeed, for the same (average) grid size $N$, the only difference in these algorithm is that in the uniform case the step-size is given by $\Delta t=T / N$, whereas in the adaptive case it has to be computed in every 
step using Lemma 4.4 That is, the only additional computational work in the adaptive case is this evaluation, which basically boils down to one evaluation of Lambert's W-function. We have used the implementation of $W$ in the GNU Scientific Library (GSL), see [9], as well as the approximation

$$
W(x) \approx \log (1+x)\left(1-\frac{\log (1+\log (1+x))}{2+\log (1+x)}\right)
$$

due to [22]. The timings from Table 4 indicate that for a one-dimensional reflected Brow-

\begin{tabular}{|c|c|c|c|c|}
\hline & Uniform & Adaptive - precise & Adaptive - approximation & Correction \\
\hline$N$ & 151 & - & - & 50 \\
\hline Work & 151 & 150.94 & 150.26 & 173.71 \\
\hline TOL & - & 0.0313 & 0.0313 & 0.4411 \\
\hline Error & 0.2281 & 0.0922 & 0.0889 & -0.0865 \\
\hline Time & 42 & 180 & 70 & 175 \\
\hline
\end{tabular}

Table 4. Running times (in seconds) on a laptop with a Intel( $R)$ Pentium( $R)$ M processor with $2.13 \mathrm{GHz}$ for problem (7.3). "Adaptive-precise" means the explicit adaptive algorithm with the precise evaluation of Lambert's W function using the GSL library, whereas "Adaptive-approximation" uses the approximation of Lambert's $W$-function. In all cases, we used $M=869200$ trajectories for the Monte-Carlo simulation. $N$ means the initial grid size, works denotes the average final grid size for the adaptive (and the uniform) algorithm and the work in the above sense for the algorithm with correction.

nian motion, the evaluation of Lambert's W-function consume almost $75 \%$ of the running time, whereas it is only $50 \%$ if one uses 7.5 ). (I.e., the approximation 7.5 ) in three times faster than the GSL-implementation.) Note that the approximation seems to be sufficiently accurate for the purposes of the algorithm. The statistical error indicator $\mathcal{S}$ is around 0.011 for all the results in Table 4

7.2. A purely reflected diffusion in dimension 2 . We present two multi-dimensional examples, one with a purely reflecting boundary and one with both reflecting and stopping boundaries. For the purely reflecting case we choose one of Gobet's examples in [13]. The process $X$ under consideration is a Brownian motion (normally) reflected on the unit-circle in dimension two, i.e., $D$ is the unit disc. We consider

$$
u(t, x)=E\left[\frac{1}{2}\left\|X_{1}\right\|^{2}+Z_{1} \mid X_{t}=x\right]
$$

which satisfies the Neumann problem

$$
\left\{\begin{aligned}
\frac{\partial}{\partial t} u(t, x) & \left.=-\frac{1}{2} \Delta u(t, x), \quad(t, x) \in\right] 0,1[\times D, \\
u(1, x) & =\frac{1}{2}\|x\|^{2}, \quad x \in D \\
\frac{\partial}{\partial n} u(t, x) & =-1, \quad(t, x) \in] 0,1[\times \partial D
\end{aligned}\right.
$$

We use both the uniform and the adaptive algorithm in its explicit form. In this case, it seems that $\beta=1.16$ is the appropriate choice for the expected number of hits at the boundary in terms of the tolerance, which would indicate a rate of weak convergence of 0.86 . Note that the partial differential equation $(7.7)$ has the exact solution

$$
u(t, x)=1-t+\frac{1}{2}\|x\|^{2},
$$


we compute $u(0,0,0)=1$. For the adaptive algorithm we have approximated the second

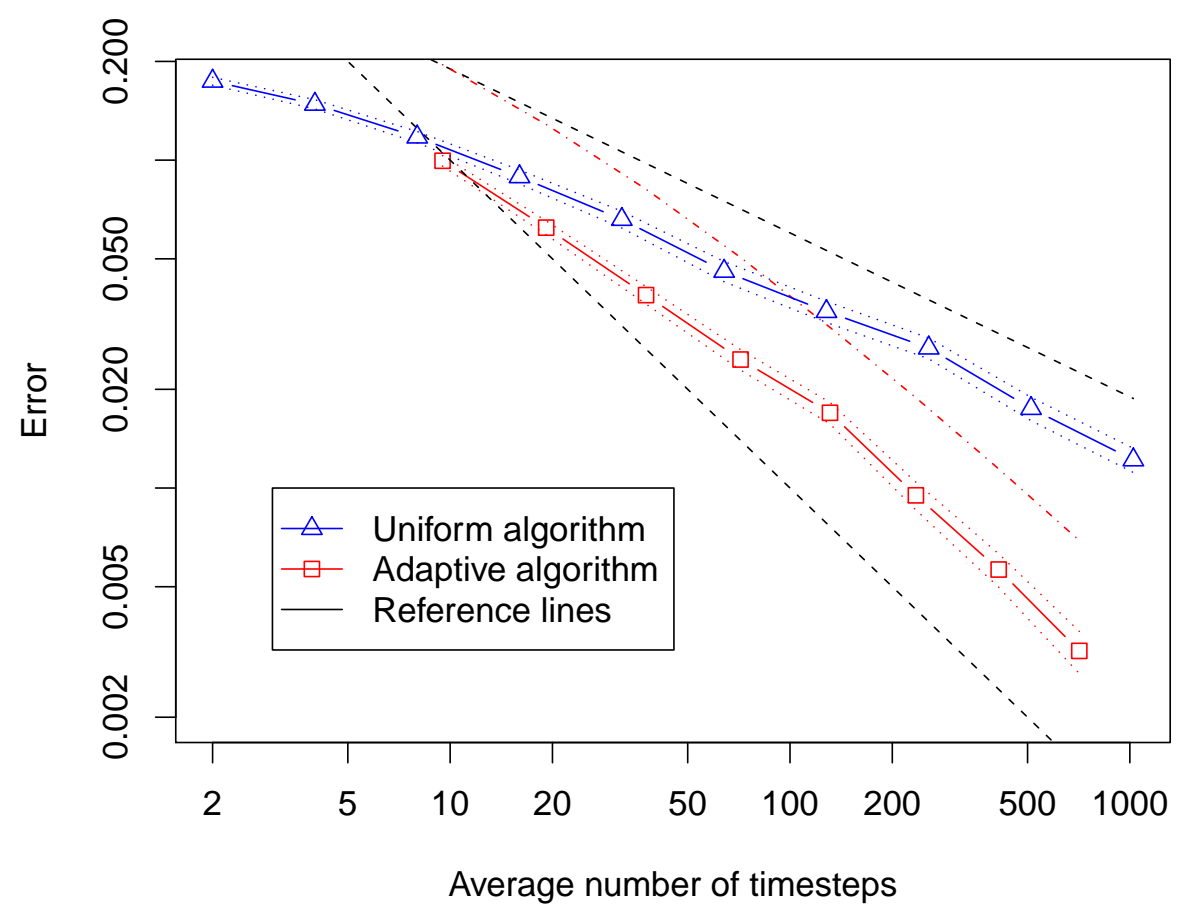

Figure 3. Absolute value of the computational error for problem $(7.7)$. The reference lines are proportional to $1 / N$ and $1 / \sqrt{N}$, respectively. The dashed-dotted line is an estimate for the leading order error term in the expansion given in Corollary 3.9 As usual, the dotted lines indicate confidence intervals around the $o b$ served errors.

\begin{tabular}{|r|r|c|c|}
\hline \multicolumn{1}{|c|}{$N$} & $M$ & Error & $\mathcal{S}$ \\
\hline 2 & 44000 & -0.1741 & 0.0048 \\
4 & 46000 & -0.1481 & 0.0048 \\
8 & 50000 & -0.1176 & 0.0046 \\
16 & 58000 & -0.0890 & 0.0044 \\
32 & 74000 & -0.0659 & 0.0039 \\
64 & 106000 & -0.0458 & 0.0032 \\
128 & 170000 & -0.0345 & 0.0026 \\
256 & 298000 & -0.0267 & 0.0020 \\
512 & 554000 & -0.0175 & 0.0014 \\
1024 & 1066000 & -0.0122 & 0.0010 \\
\hline
\end{tabular}

Table 5. Results of the uniform algorithm for problem (7.7).

normal derivative $u_{n n}$ by the constant 1 , which incidentally is the correct value in this special example.The fitted order of convergence of the adaptive algorithm is 0.78 , which improves to 0.9 if the first three observations tolerance levels are discarded. 


\begin{tabular}{|c|r|r|c|c|}
\hline TOL & \multicolumn{1}{|c|}{$N$} & \multicolumn{1}{c|}{$M$} & \multicolumn{1}{c|}{ Error } & $\mathcal{S}$ \\
\hline 0.3906 & 9.50 & 82000 & -0.0996 & 0.0037 \\
0.2441 & 19.17 & 122000 & -0.0622 & 0.0030 \\
0.1526 & 37.71 & 202000 & -0.0388 & 0.0024 \\
0.0954 & 71.54 & 362000 & -0.0247 & 0.0018 \\
0.0596 & 131.11 & 682000 & -0.0170 & 0.0013 \\
0.0373 & 234.63 & 1322000 & -0.0095 & 0.0009 \\
0.0233 & 411.14 & 2602000 & -0.0056 & 0.0007 \\
0.0146 & 709.82 & 5162000 & -0.0032 & 0.0005 \\
\hline
\end{tabular}

Table 6. Results of the adaptive algorithm for problem (7.7).

While the order of convergence for the adaptive scheme does not seem to be far away from 1, Gobet's algorithm still seems to be much more efficient in this case. Indeed, Gobet reports a computational error of 0.0038 already for only $N=10$ steps of his half-space algorithm, while the adaptive algorithm needs about $N=700$ (in the average) to achieve a similar result.

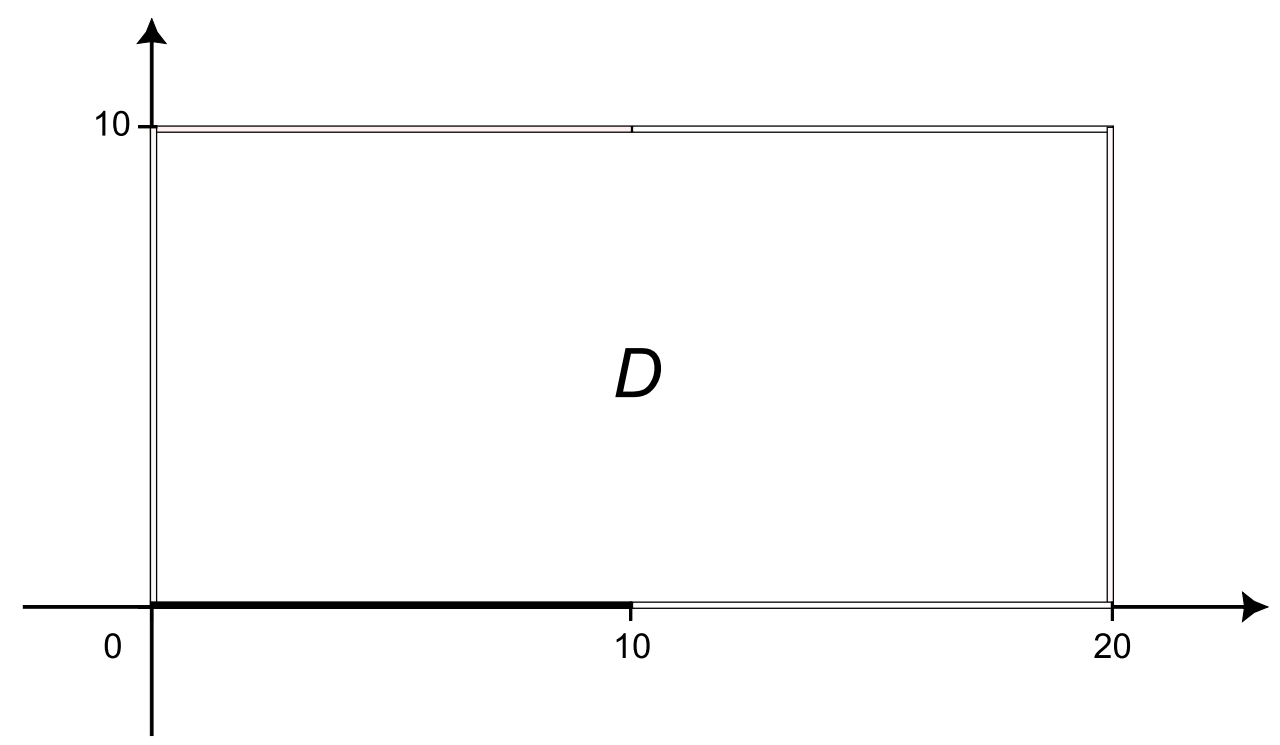

Figure 4. Domain for the two-dimensional example with mixed boundary conditions. The solid line denotes the reflecting boundary, the "empty" lines belong to the stopping boundary.

7.3. A stopped reflected diffusion in dimension 2. The second example in dimension two is a Neumann and Dirichlet boundary value problem, see equation 11.3 for the general framework. More precisely, let $D=] 0,20[\times] 0,10\left[\right.$, and let $\left.\partial D_{R}=\right] 0,10[\times\{0\}$ be the 
Neumann boundary and $\partial D_{S}=\partial D \backslash \partial D_{R}$, see Figure 4 . Consider the problem

$$
\left\{\begin{aligned}
\frac{\partial}{\partial t} u(t, x) & =-\frac{1}{2} \Delta u(t, x), \quad(t, x) \in[0,2] \times D, \\
u(2, x) & =10 \exp \left(-\sqrt{\left(10-x_{1}\right)^{2}+x_{2}^{2}}\right), \quad x \in D, \\
\frac{\partial}{\partial n} u(t, x) & =x_{1}, \quad(t, x) \in[0,2] \times \partial D_{R}, \\
u(t, x) & =10 \exp \left(-\sqrt{\left(10-x_{1}\right)^{2}+x_{2}^{2}}\right), \quad(t, x) \in[0,2] \times \partial D_{S} .
\end{aligned}\right.
$$

Notice that the solution $u(t, x)$ of $(7.8)$ has singularities at $x=(0,0)$ and at $x=(10,0)$, where the Neumann and the Dirichlet boundaries meet. The stochastic representation is given by a Brownian motion $X_{t}$ reflected at $\partial D_{R}$ and stopped when hitting $\partial D_{S}$, see Proposition 1.10 Once again, we compute $u\left(0, x_{0}\right)$ with $x_{0}=(10,0.2)$, close to the singularity at $(10,0)$. Using the commercial finite-element package FEMLAB, see [4], we have computed

$$
u(0,10,0.2) \approx 4.352,
$$

which is used as reference value for the reported computational errors.

Since the stochastic representation of Proposition 1.10 involves sampling from a mixed reflected-stopped diffusion, we use the uniform Algorithm 2.1 and the adaptive Algorithm 5.2 for the case of both Neumann and Dirichlet boundary conditions.

\begin{tabular}{|r|r|c|c|r|}
\hline \multicolumn{1}{|c|}{$N$} & \multicolumn{1}{|c|}{$M$} & Error & $\mathcal{S}$ & $N_{\text {tot }}$ \\
\hline 2 & 20020 & -3.3371 & 0.0643 & 1.79 \\
4 & 20040 & -3.1662 & 0.0681 & 3.18 \\
8 & 20080 & -2.7336 & 0.0687 & 5.63 \\
16 & 20160 & -2.2720 & 0.0715 & 10.04 \\
32 & 20320 & -1.7794 & 0.0710 & 18.12 \\
64 & 20640 & -1.2920 & 0.0698 & 33.39 \\
128 & 21280 & -1.0527 & 0.0698 & 63.16 \\
256 & 22560 & -0.7367 & 0.0673 & 118.4 \\
512 & 25120 & -0.5640 & 0.0639 & 228.9 \\
1024 & 30240 & -0.4172 & 0.0581 & 445.7 \\
2048 & 40480 & -0.2975 & 0.0505 & 861.2 \\
4096 & 60960 & -0.1921 & 0.0404 & 1704 \\
8192 & 101920 & -0.1617 & 0.0315 & 3357 \\
16384 & 183840 & -0.1311 & 0.0236 & 6669 \\
32768 & 347680 & -0.0878 & 0.0171 & 13247 \\
65536 & 675360 & -0.0556 & 0.0122 & 26331 \\
131072 & 1330720 & -0.0342 & 0.0087 & 52427 \\
\hline
\end{tabular}

Table 7. Results of the uniform Euler Monte-Carlo algorithm for problem (7.8) with $x_{0}=(10,0.2)$.

Table 7 gives the results of the uniform Euler scheme for equation (7.8). While $N$ denotes the size of the uniform grid, $N_{\text {tot }}$ denotes the average grid size until the algorithm stops. The iteration only needs to be computed until the first (observed) hitting time of the discretized process at the stopping boundary. Note that Table 7 shows that the uniform Euler scheme still seems to converge with order $1 / 2$ in the singular situation 7.8 , even though the starting vector $x_{0}=(10,0.2)$ is close to one singularity of the solution $u$. Indeed, a numerical estimate gives an estimated order of convergence of 0.46 with respect to $N_{\text {tot }}$ 
provided that the first four results of Table 7 are discarded and similar results hold for convergence with respect to $N$.

For the adaptive algorithm, we use Algorithm 5.2 with

$$
\left|u_{n n}\left(t_{i}, \bar{X}_{i}^{\mathrm{t}}\right)\right|=\frac{1}{\left\|\bar{X}_{i}^{\mathrm{t}}-x_{\text {sing }}\right\|^{\eta}+\mathrm{TOL}^{\alpha}},
$$

where $x_{\text {sing }}=(10,0)$, the place of one singularity of $u$, and $\alpha$ and $\eta$ are two parameters. The second parameter, $\eta$ gives the order of the singularity of $u$ at $x_{\text {sing. }}$. We use $\eta=1 / 2$, even though actual computation of $u_{n n}$ using a finite element algorithm shows that the singularity is more complicated. We have also used $\eta=0$, which corresponds to not using

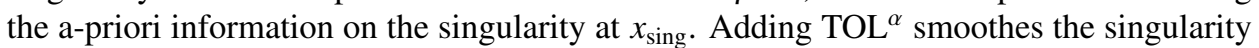
out, we choose $\alpha=2$.

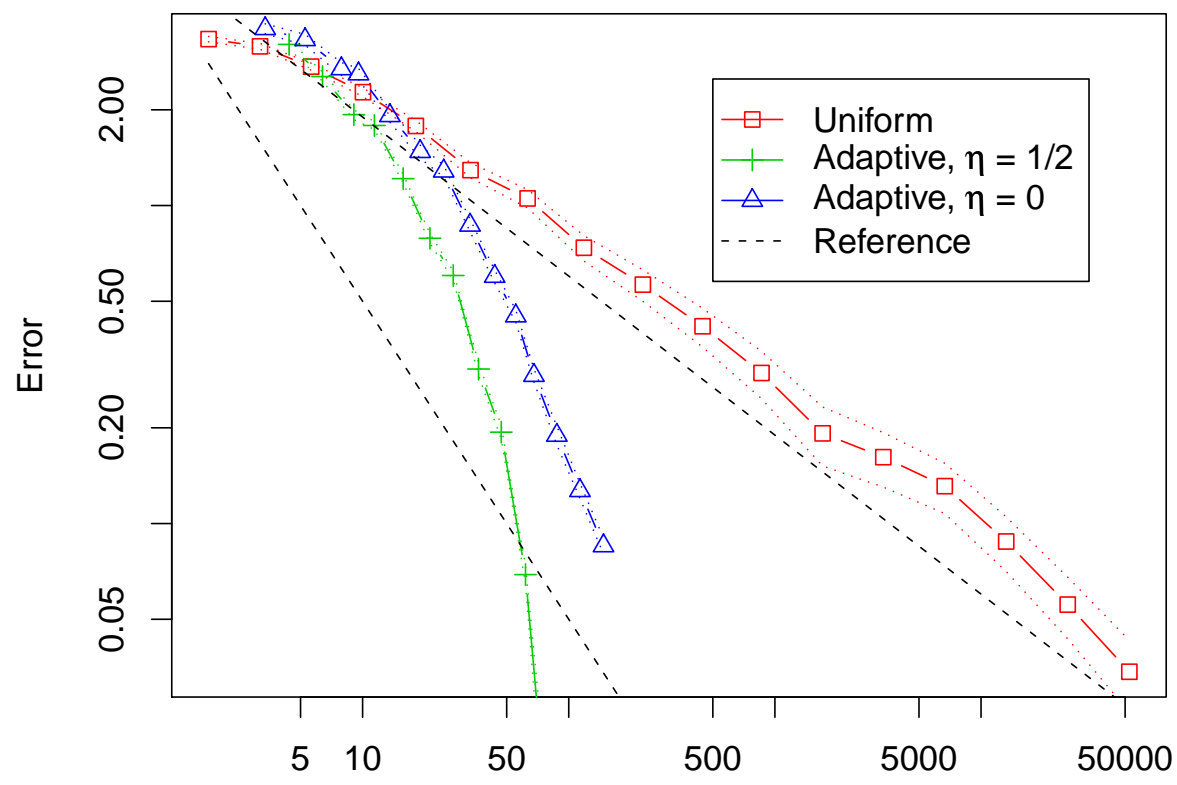

Average number of time-steps

Figure 5. Absolute value of the error for problem $(7.8)$ for $x_{0}=(10,0.2)$. The reference lines are proportional to $1 / N$ and $1 / \sqrt{N}$, respectively. The dashed line in color is proportional to the error estimate computed for the refinement algorithm, whereas the dotted lines correspond to $90 \%$ confidence intervals around the error.

The empirical rates of convergence for the results of the adaptive Euler algorithm with $\eta=0$ and with $\eta=1 / 2$ are both larger than 1 . The explanation for this apparent contradiction with the previous results is a very favorable cancellation of error contribution from the stopped and the reflected diffusion. Indeed, what we see in these results is mainly the decrease of the error of a purely stopped Brownian motion. The error coming from the reflection only materializes at smaller levels of TOL, and, as we strongly expect, will slow down the asymptotic order of convergence to the reasonable usual levels. Unfortunately, we cannot convincingly show this effect in the plot due to the statistical error.

As a contrast, we have found out that only a slight change of $x_{0}$ can already change the situation completely. Here, the error cancellations are so unfavorable that the adaptive 


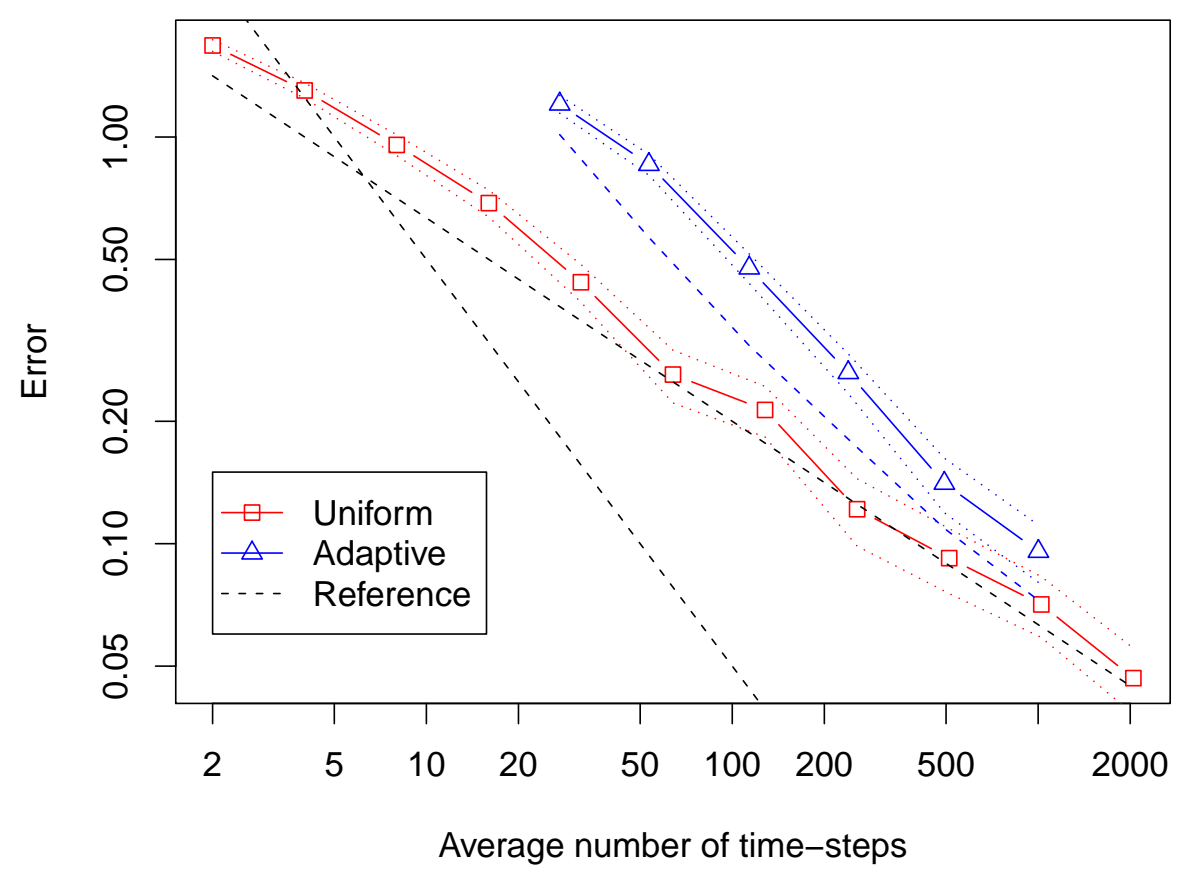

Figure 6. Absolute value of the error for problem 7.8$)$ at $x_{0}=(9.5,0.1)$. The reference lines are proportional to $1 / \sqrt{N}$ and $1 / N$, respectively.

algorithm performs even worse than the uniform algorithm. Note, however, that we still get an empirical weak rate of convergence of 0.75 for the adaptive algorithm, which is in line with our expectations (of a rate of around 0.8). In contrast, the empirical rate of the uniform Euler algorithm in this case is 0.54 .

\begin{tabular}{|c|r|r|c|c|}
\hline TOL & \multicolumn{1}{|c|}{$N$} & \multicolumn{1}{|c|}{$M$} & Error & $\mathcal{S}$ \\
\hline 0.3086 & 4.40 & 9000 & -3.2112 & 0.1024 \\
0.1715 & 6.39 & 13000 & -2.5444 & 0.0848 \\
0.0953 & 9.07 & 21000 & -1.9312 & 0.0680 \\
0.0529 & 11.41 & 37000 & -1.7854 & 0.0511 \\
0.0294 & 15.73 & 69000 & -1.2149 & 0.0375 \\
0.0163 & 21.22 & 133000 & -0.7889 & 0.0273 \\
0.0091 & 27.52 & 261000 & -0.6025 & 0.0194 \\
0.0050 & 36.51 & 517000 & -0.3061 & 0.0138 \\
0.0028 & 47.06 & 1029000 & -0.1938 & 0.0098 \\
0.0016 & 61.68 & 5125000 & -0.0691 & 0.0044 \\
0.0009 & 78.06 & 10245000 & -0.0124 & 0.0031 \\
\hline
\end{tabular}

Table 8. Results of the adaptive Euler algorithm with $\alpha=2$ and $\eta=1 / 2$ for problem (7.8).

We have plotted the spacial distribution of the triggered refinements in Figure 7 Note that whether the grid is refined at some time $t_{i}$ or not only depends on the position of $\bar{X}_{i}$ 


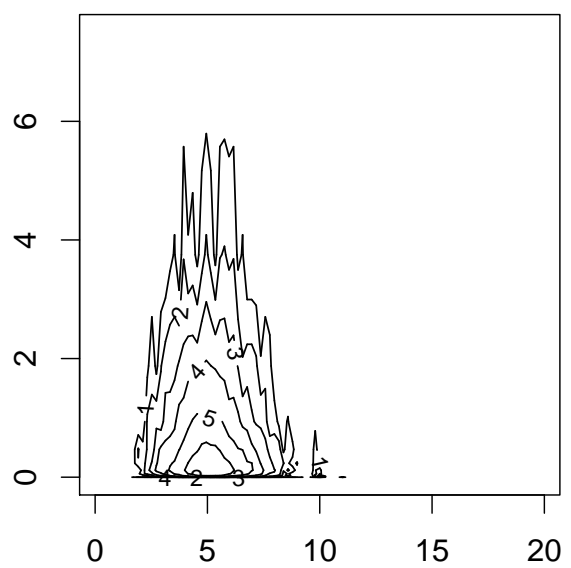

(a) Refinements with $l^{(\text {ref })}>l^{(\text {stop })}$.

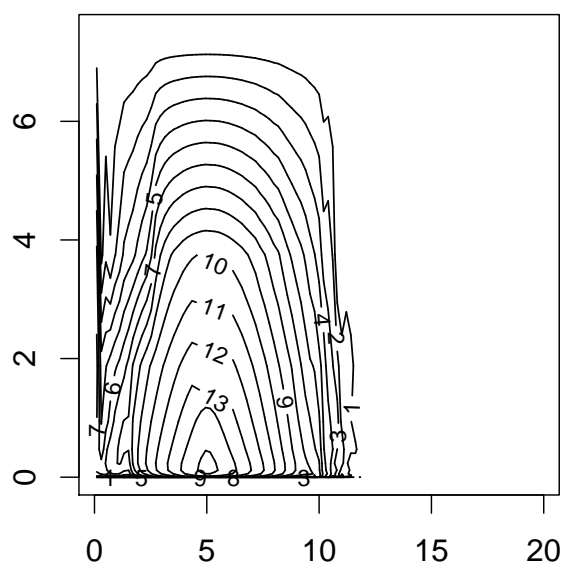

(b) Refinements with $l^{(\text {ref })}<l^{(\text {stop })}$.

Figure 7. Locations of refinements for $x_{0}=(5,0.5)$. The figures display the number of refinements over a subdivision of the domain into rectangles. The number of refinements are given on a logarithmic scale.

(and of $\bar{X}_{i+1}$ for the stopped error contribution). Therefore, it is meaningful to collect all the positions $x \in D$, where refinements occur and count the frequencies of those refinements. In order to being able to distinguish the starting point of the process from the singularity $(10,0)$, we have chosen $x_{0}=(5,0.5)$ for this purpose. Note that most refinements are actually due to the error contribution of the stopped diffusion, even far away from $\partial D_{S}$.

\section{Conclusions}

In this work, we have considered the weak approximation problem for stochastic differential equations, which are reflected at the boundary of a domain $D$ and, possibly, stopped at some part of the boundary. We have developed a new error representation for the approximation of expected values of functionals depending on the solution, whose computable leading order term can be decomposed into four different contributions:

(1) the classical "interior" error from the Euler scheme in the interior of a domain as in [21] and [20],

(2) an error contribution caused by mis-specification of the stopping time similar to [7],

(3) an error contribution due to discrete reflection at the boundary determined by the second normal derivative of the solution $u$ of the Kolmogorov backward equation at the reflecting boundary,

(4) an new additional term due to the interplay between reflection and stopping conditions.

We have used this error representation as the basis for an adaptive weak projected Euler scheme for reflected diffusions. In the case of a stopped reflected diffusion, the fourth term in our error representation is, however, neglected for the grid-refinement algorithm applied in our numerical example.

The above mentioned adaptive Euler scheme is believed to converge with a weak rate of approximately 0.8 . This result is motivated by a combination of numerical results, relating the tolerance level TOL of the adaptive scheme to the expected number of hits at the reflecting boundary, and theoretical results, relating the expected number of hits at the boundary to the convergence rate. Moreover, an alternative algorithm for one-dimensional 
problems only indicates that the above adaptive algorithm might be asymptotically optimal for the projected Euler scheme among all adaptive algorithms solely based on the leading order term of our error expansion. We have also presented several numerical examples, in dimensions one and two, backing our findings.

In this work, we have only considered the time-discretization error for the weak approximation problem, i.e., we have completely neglected the corresponding integration problem - which we solve by Monte-Carlo simulation. Usually, the integration error is, however, at least as important as the time-discretization error.

As a future next step, we will explore possible complexity improvements using Giles' [10] multi-level Monte-Carlo method, both for the uniform and for the adaptive projected Euler algorithms. This requires to additionally consider the strong approximation problem for stopped reflected diffusions. Note that multi-level approximation has already been extended to adaptive Euler schemes for ordinary and stopped diffusions [cite your work]. Therefore, we will mainly need to treat the case of stopped reflected diffusions.

\section{Acknowledgments.}

This work was partially supported by the Dahlquist fellowship at the Royal Institute of Technology in Stockholm, Sweden, the department of Scientific Computing in Florida State University and the VR project "Effektiva numeriska metoder för stokastiska differentialekvationer med tillämpningar".

\section{REFERENCES}

[1] Milton Abramowitz and Irene A. Stegun. Handbook of mathematical functions with formulas, graphs, and mathematical tables, volume 55 of National Bureau of Standards Applied Mathematics Series. For sale by the Superintendent of Documents, U.S. Government Printing Office, Washington, D.C., 1964.

[2] Søren Asmussen, Peter Glynn, and Jim Pitman. Discretization error in simulation of one-dimensional reflecting Brownian motion. Ann. Appl. Probab., 5(4):875-896, 1995.

[3] Mireille Bossy, Emmanuel Gobet, and Denis Talay. A symmetrized Euler scheme for an efficient approximation of reflected diffusions. J. Appl. Probab., 41(3):877-889, 2004.

[4] COMSOL. FEMLAB Version 2.3, Reference manual, 2003.

[5] R. M. Corless, G. H. Gonnet, D. E. G. Hare, D. J. Jeffrey, and D. E. Knuth. On the Lambert $W$ function. Adv. Comput. Math., 5(4):329-359, 1996.

[6] C. Costantini, B. Pacchiarotti, and F. Sartoretto. Numerical approximation for functionals of reflecting diffusion processes. SIAM J. Appl. Math., 58(1):73-102 (electronic), 1998.

[7] Anna Dzougoutov, Kyoung-Sook Moon, Erik von Schwerin, Anders Szepessy, and Raúl Tempone. Adaptive Monte Carlo algorithms for stopped diffusion. In Multiscale methods in science and engineering, volume 44 of Lect. Notes Comput. Sci. Eng., pages 59-88. Springer, Berlin, 2005.

[8] Mark Freidlin. Functional integration and partial differential equations, volume 109 of Annals of Mathematics Studies. Princeton University Press, Princeton, NJ, 1985.

[9] Mark Galassi, Jim Davies, James Theiler, Brian Gough, Gerard Jungman, Michael Booth, and Fabrice Rossi. Gnu Scientific Library: Reference Manual. Network Theory Ltd., February 2003.

[10] Michael B. Giles. Multilevel Monte Carlo path simulation. Oper. Res., 56(3):607-617, 2008.

[11] Paul Glasserman. Monte Carlo methods in financial engineering, volume 53 of Applications of Mathematics (New York). Springer-Verlag, New York, 2004. Stochastic Modelling and Applied Probability.

[12] Emmanuel Gobet. Weak approximation of killed diffusion using Euler schemes. Stochastic Process. Appl., 87(2):167-197, 2000

[13] Emmanuel Gobet. Euler schemes and half-space approximation for the simulation of diffusion in a domain. ESAIM Probab. Statist., 5:261-297 (electronic), 2001.

[14] Ioannis Karatzas and Steven E. Shreve. Brownian motion and stochastic calculus. 2nd ed. Graduate Texts in Mathematics, 113. New York etc.: Springer-Verlag. , 1991.

[15] Peter E. Kloeden and Eckhard Platen. Numerical solution of stochastic differential equations, volume 23 of Applications of Mathematics (New York). Springer-Verlag, Berlin, 1992.

[16] Kyong-Sook Moon, Anders Szepessy, Raúl Tempone, and Georgios E. Zouraris. Convergence rates for adaptiv weak approximation of stochastic differential equations. Stochastic Analysis and Applications, 23:511-558, 2005.

[17] Daniel Revuz and Marc Yor. Continuous martingales and Brownian motion. 3rd ed., 3rd. corrected printing. Grundlehren der Mathematischen Wissenschaften 293. Berlin: Springer., 2005.

[18] Yasumasa Saisho. Stochastic differential equations for multidimensional domain with reflecting boundary. Probab. Theory Related Fields, 74(3):455-477, 1987. 
[19] Leszek Słomiński. Euler's approximations of solutions of SDEs with reflecting boundary. Stochastic Process. Appl., 94(2):317-337, 2001.

[20] Anders Szepessy, Raúl Tempone, and Georgios E. Zouraris. Adaptive weak approximation of stochastic differential equations. Comm. Pure Appl. Math., 54(10):1169-1214, 2001.

[21] Denis Talay and Luciano Tubaro. Expansion of the global error for numerical schemes solving stochastic differential equations. Stochastic Anal. Appl., 8(4):483-509 (1991), 1990.

[22] Serge Winitzki. Uniform approximations for transcendental functions. In Computational science and its applications-ICCSA 2003. Part I, volume 2667 of Lecture Notes in Comput. Sci., pages 780-789. Springer, Berlin, 2003.

\section{APPENDIX A. AN UPPER BOUND FOR HITTING PROBABILITIES}

In order to effectively use the error expansion given in Proposition 3.5 , we need to estimate $P\left(t_{i} \leq \hat{\tau}<t_{i+1} \mid \mathcal{X}\right)$. Let us denote the conditional probability given $\mathcal{X}$ by $P^{X}$. Moreover, let $\hat{\tau}_{i}$ denote the first hitting time of $\hat{X}$ at $\partial D_{S}$ after time $t_{i}, i=0, \ldots, N-1$. By the Markov property, we have

$$
P^{X}\left(t_{i} \leq \hat{\tau}<t_{i+1}\right)=P^{X}\left(t_{i} \leq \hat{\tau}<t_{i+1} \mid \hat{\tau} \geq t_{i}\right) P^{X}\left(\hat{\tau} \geq t_{i}\right)=P^{X}\left(\hat{\tau}_{i}<t_{i+1}\right) P^{X}\left(\hat{\tau} \geq t_{i}\right) .
$$

Furthermore, $P^{X}\left(\hat{\tau}<t_{i}\right)$ can be iteratively computed by

$$
\begin{aligned}
P^{X}\left(\hat{\tau}<t_{i}\right) & =P^{X}\left(\hat{\tau}<t_{i} \mid \hat{\tau}<t_{i-1}\right) P^{X}\left(\hat{\tau}<t_{i-1}\right)+P^{X}\left(\hat{\tau}<t_{i} \mid \hat{\tau} \geq t_{i-1}\right) P^{X}\left(\hat{\tau} \geq t_{i-1}\right) \\
& =P^{X}\left(\hat{\tau}<t_{i-1}\right)+P^{X}\left(\hat{\tau}_{i-1}<t_{i}\right)\left(1-P^{X}\left(\hat{\tau}<t_{i-1}\right)\right) .
\end{aligned}
$$

Since $P^{X}\left(\hat{\tau}<t_{1}\right)=P^{X}\left(\hat{\tau}_{0}<t_{1}\right)$, this leaves us with the task to approximate $P^{X}\left(\hat{\tau}_{i-1}<\right.$ $\left.t_{i}\right), i=1, \ldots, N$, which is the probability of hitting $\partial D_{S}$ for a Brownian bridge between $x=\hat{X}_{t_{i-1}}$ and $y=\hat{X}_{t_{i}}$ between $t_{i-1}$ and $t_{i}$. Notice that hitting probabilities of Brownian bridges decrease exponentially with $\operatorname{dist}(x, D) \wedge \operatorname{dist}(y, D)$. Therefore, we may assume that the boundary $D$ is given by a hyperplane - e.g., by a tangent plane to the true boundary. Moreover, assume that the boundary between $\partial D_{S}$ and $\partial D_{R}$ is given by $\partial D \cap H$ for some hyperplane $H$. Without loss of generality, we choose coordinates such that

$$
\partial D=\left\{\xi \in \mathbb{R}^{d} \mid\left\langle\xi, n_{D}\right\rangle=c_{D}\right\}, \quad H=\left\{\xi \in \mathbb{R}^{d} \mid\left\langle\xi, n_{H}\right\rangle=c_{H}\right\}
$$

with $\xi \in D_{S}$ iff $\left\langle\xi, n_{D}\right\rangle \leq c_{D}$ and $\left\langle\xi, n_{H}\right\rangle \geq c_{H}$. Then we approximate

$$
P^{X}\left(\hat{\tau}_{i-1}<t_{i}\right) \leq P^{X}\left(\inf _{t_{i-1} \leq s<t_{i}}\left\langle\hat{X}_{s}, n_{D}\right\rangle \leq c_{D}\right) \times P^{X}\left(\sup _{t_{i-1} \leq s<t_{i}}\left\langle\hat{X}_{s}, n_{H}\right\rangle \geq c_{H}\right) .
$$

This formula is, however, only used for $\hat{X}_{t_{i-1}} \in D$ and $\hat{X}_{t_{i}} \in D$. If both of them are outside $D$, we replace the first factor by the probability to hit the boundary from outside, i.e., we set

$$
P^{X}\left(\hat{\tau}_{i-1}<t_{i}\right) \leq P^{X}\left(\inf _{t_{i-1} \leq s<t_{i}}\left\langle\hat{X}_{s}, n_{D}\right\rangle \geq c_{D}\right) \times P^{X}\left(\sup _{t_{i-1} \leq s<t_{i}}\left\langle\hat{X}_{s}, n_{H}\right\rangle \geq c_{H}\right) .
$$

Moreover, if precisely one of the points $\hat{X}_{t_{i-1}}$ and $\hat{X}_{t_{i}}$ is outside $D$ and the other one is inside $D$, then $\partial D$ must have been crossed and we use the bound

$$
P^{X}\left(\hat{\tau}_{i-1}<t_{i}\right) \leq P^{X}\left(\sup _{t_{i-1} \leq s<t_{i}}\left\langle\hat{X}_{s}, n_{H}\right\rangle \geq c_{H}\right) .
$$

We have now effectively reduced the problem to a one-dimensional. Indeed, let $Y$ denote a (one-dimensional) Brownian bridge with $Y_{0}=y_{0}$ and $Y_{\Delta t}=y_{1}$ coming from a Brownian motion with quadratic variation $\sigma^{2} t$, then, using the notation $(x)_{+}=x \vee 0$,

$$
P\left(\sup _{0 \leq t<\Delta t} Y_{t} \geq \lambda\right)=\exp \left(-2 \frac{\left(\lambda-y_{0}\right)_{+}\left(\lambda-y_{1}\right)_{+}}{\sigma^{2} \Delta t}\right)
$$

see, for instance, [7]. We apply this result for the Brownian bridges $\left\langle\hat{X}_{t}, n_{D}\right\rangle$ and $\left\langle\hat{X}_{t}, n_{H}\right\rangle$, respectively, where $t_{i-1} \leq t<t_{i}$ and we condition on the $\sigma$-algebra $\mathcal{X}$. This finally gives 
the computable approximation

$$
\begin{aligned}
P^{X}\left(\hat{\tau}_{i-1}<t_{i}\right) \leq \exp \left(-2 \frac{\left(\left\langle n_{D}, \hat{X}_{t_{i-1}}\right\rangle-c_{D}\right)_{+}\left(\left\langle n_{D}, \hat{X}_{t_{i}}\right\rangle-c_{D}\right)_{+}}{\hat{\sigma}_{D}^{2} \Delta t_{i-1}}\right) \times \\
\quad \times \exp \left(-2 \frac{\left(c_{H}-\left\langle n_{H}, \hat{X}_{t_{i-1}}\right\rangle\right)_{+}\left(c_{H}-\left\langle n_{H}, \hat{X}_{t_{i}}\right\rangle\right)_{+}}{\hat{\sigma}_{H}^{2} \Delta t_{i-1}}\right),
\end{aligned}
$$

where

$$
\hat{\sigma}_{D}^{2}=\sum_{j=1}^{d}\left\langle V_{j}\left(\hat{X}_{t_{i-1}}\right), n_{D}\right\rangle^{2}, \quad \hat{\sigma}_{H}^{2}=\sum_{j=1}^{d}\left\langle V_{j}\left(\hat{X}_{t_{i-1}}\right), n_{H}\right\rangle^{2} .
$$

Again, A.4 only holds if both $\hat{X}_{t_{i-1}}$ and $\hat{X}_{t_{i}}$ are inside the domain $D$. Otherwise we adjust A.4 according to A.3.

Note that we also need a lower bound for $P^{X}\left(\hat{\tau}_{i-1}<t_{i}\right)$ in order to get an upper bound for (A.1). For simplicity, we approximate a lower bound by setting

$$
P^{X}\left(\hat{\tau}_{i-1}<t_{i}\right) \lesssim c P_{\text {up }}^{X} \text {, }
$$

where $P_{\text {up }}^{X}$ denotes the upper bound computed in A.4 and $0<c<1$ is a fixed constant.

Remark A.1. Notice that the approximation error in A.4 can be considerable, depending on the location relative to the boundary. If the points $\hat{X}_{t_{i-1}}$ and $\hat{X}_{t_{i}}$ are far away from the separating hyperplane $H$, but near to the stopping boundary $\partial D_{S}$, the error will be small. On the other hand, if the points are both near to $H$ and to $\partial D$, then the error will be rather high. On the other hand, the approximation clearly shows the exponential decay of the hitting probabilities with distance from the stopping boundary.

Institute for Mathematics, Royal Institute of Technology, S-10044 Stockholm, Sweden

E-mail address: cbayer@kth.se

Institute for Mathematics, Royal Institute of Technology, S-10044 Stockholm, Sweden

E-mail address: szepessy@kth. se

Applied Mathematics and Computational Sciences, KaUST, Thuwal, Saudi Arabia

E-mail address: raul . tempone@kaust.edu.sa 